\title{
Quantification of 3D cardiac wall deformation using magnetic resonance tagging
}

Citation for published version (APA):

Aelen, F. W. L. (1999). Quantification of 3D cardiac wall deformation using magnetic resonance tagging. [Doctoral Thesis, Maastricht University]. Universiteit Maastricht. https://doi.org/10.26481/dis.19991007fa

Document status and date:

Published: 01/01/1999

DOI:

10.26481/dis.19991007fa

Document Version:

Publisher's PDF, also known as Version of record

\section{Please check the document version of this publication:}

- A submitted manuscript is the version of the article upon submission and before peer-review. There can be important differences between the submitted version and the official published version of record.

People interested in the research are advised to contact the author for the final version of the publication, or visit the DOI to the publisher's website.

- The final author version and the galley proof are versions of the publication after peer review.

- The final published version features the final layout of the paper including the volume, issue and page numbers.

Link to publication

\footnotetext{
General rights rights.

- You may freely distribute the URL identifying the publication in the public portal. please follow below link for the End User Agreement:

www.umlib.nl/taverne-license

Take down policy

If you believe that this document breaches copyright please contact us at:

repository@maastrichtuniversity.nl

providing details and we will investigate your claim.
}

Copyright and moral rights for the publications made accessible in the public portal are retained by the authors and/or other copyright owners and it is a condition of accessing publications that users recognise and abide by the legal requirements associated with these

- Users may download and print one copy of any publication from the public portal for the purpose of private study or research.

- You may not further distribute the material or use it for any profit-making activity or commercial gain

If the publication is distributed under the terms of Article $25 \mathrm{fa}$ of the Dutch Copyright Act, indicated by the "Taverne" license above, 


\title{
QUANTIFICATION OF 3D CARDIAC WALL DEFORMATION USING MAGNETIC RESONANCE TAGGING
}

\author{
PROEFSCHRIFT
}

ter verkrijging van de graad van doctor aan de Universiteit Maastricht, op gezag van de Rector Magnificus,

Prof. dr. A.C. Nieuwenhuijzen Kruseman, volgens het besluit van het College van Decanen, in het openbaar te verdedigen op donderdag 7 oktober 1999 om 16.00 uur

$$
\text { door }
$$

Frank Willem Lambertus Aelen

geboren te Tegelen op 30 november 1968 
Promotores:

Prof. dr. ir. T. Arts

Prof. dr. R.S. Reneman

Co-promotor.

Dr. F.W. Prinzen

Beoordelingscommissie:

Prof. dr. L. Snoeckx (voorzitter)

Prof. dr. ir. D.H. van Campen (Technische Universiteit Eindhoven)

Dr. E. Cheriex

Prof. dr. J.M.A. van Engelshoven

Prof. dr. K. Nicolay (Universiteit Utrecht)

This thesis comprises studies carried out in the department of Biophysics of the Cardiovascular Research Institute Maastricht (CARIM), University Maastricht, Maastricht, The Netherlands.

Financial support by the Netherlands Heart Foundation for the publication of this thesis is gratefully acknowledged.

(C) 1999 F.W.L. Aelen, Maastricht, The Netherlands

All rights reserved. No part of this publication may be reproduced or transmitted in any form or by any means, electronic or mechanical, including photocopy, recording or any information storage or retrieval system, without permission in writing from the copyright owner. 


\section{Contents}

Chapter 1 General introduction

Chapter 2 Kinematic analysis of left ventricular deformation in myocardial infarction using Magnetic Resonance cardiac tagging

Chapter 3 Relation between torsion and cross-sectional area change in the human left ventricle

Chapter 4 Quantification of 3D left ventricular deformation with a kinematic model using Magnetic Resonance cardiac tagging

Chapter 5 Automated quantification of 3D deformation of the healthy and infarcted left ventricle using Magnetic Resonance cardiac tagging

Chapter 6 General discussion

Summary

Samenvatting 



\section{Chapter 1}

General introduction 


\subsection{INTRODUCTION}

The primary role of the heart is to pump blood through the vascular system by proper cyclic contraction and relaxation. In clinical diagnosis of cardiac pathologies information has to be obtained about the ability of the myocardial tissue to contract and relax. This ability is, amongst other things, reflected in the way the myocardial wall deforms during the cardiac cycle. Experimental studies (Tyberg et al., 1974; Gallagher et al., 1982; Prinzen et al., 1984; Waldman and Covell, 1987; Prinzen et al., 1989), generally using invasive techniques performed on animals, have shown that deformation of the heart wall is very sensitive to regional myocardial dysfunction. Therefore, noninvasive methods to obtain information about the deformation of the heart wall during the cardiac cycle have become increasingly important in the clinic. Currently, echocardiography and Magnetic Resonance Imaging (MRI) are used clinically to analyze motion and deformation of heart wall contours. Information is obtained, for example, about left ventricular ejection fraction, changes in wall thickness during the cardiac cycle, and wall curvature. More recently, the advent of Magnetic Resonance (MR) cardiac tagging (Zerhouni et al., 1988; Axel and Dougherty, 1989; McVeigh, 1996) enabled noninvasive quantitative analysis of threedimensional (3D) tissue deformation within the heart wall. However, the technique is not yet in use in the clinic routinely, due to the still laborious methods needed to analyze the acquired images.

In the studies presented in this thesis methods are developed to quantify deformation of the left ventricle during the cardiac cycle using MR cardiac tagging. This introductory chapter starts with a description of the relevant cardiac anatomy and function. Subsequently, techniques to measure cardiac wall deformation are introduced. Influences of cardiac pathologies on left ventricular deformation and their relevance for clinical diagnostics are addressed. Next, the basic physical principles of MR imaging and MR cardiac tagging are discussed briefly. An overview of current methods to analyze MR tagged images is given. The strengths and shortcomings of these methods are discussed, giving rise to the aims of the thesis. The chapter is concluded with an outline of the thesis. 


\subsection{BASIC CARDIAC ANATOMY AND FUNCTION}

This section is confined to a brief description of the anatomical and functional aspects of the heart that are relevant in relation to the thesis. The information presented is basic and is derived from textbooks (Guyton, 1991).

The heart is a hollow muscular structure which serves primarily to pump blood through the vascular system. It actually consists of two pumps which are functionally in series. The right part of the heart pumps the deoxygenated blood from the peripheral organs to the lungs, while the left part of the heart pumps the oxygenated blood from the lungs to the peripheral organs. Both parts of the heart are composed of an atrium and a ventricle. In the diastolic phase of the cardiac cycle, in which the cardiac tissue is relaxed, the ventricles fill with blood. The right heart part fills with blood coming from the peripheral organs, while the left heart part fills with blood coming from the lungs. Just prior to the systolic phase the atria contract, which completes filling of the ventricles. While the ventricles start to contract the valves between atria and ventricles close. The pressures in the left and right ventricles increase until they exceed the pressures in their respective outflow arteries, i.e., the aorta and the pulmonary artery, respectively. Subsequently, ejection of blood from the ventricles begins. After relaxation of the cardiac tissue the pressures in the ventricles drop and ejection stops. Subsequently, the diastolic phase begins again.

This study deals with the quantification of deformation of the cardiac left ventricle during systole. The geometry of the left ventricle resembles a thick-walled truncated prolate ellipsoid with short and long axes (figure 1.1). At the tip of the left ventricle the apex is located. On the opposite side, where the mitral and aortic valves are attached to the left ventricular wall, the base is located. The mitral valve is the valve between left atrium and left ventricle, while the aortic valve is the valve between left ventricle and aorta. Papillary muscies connect the mitral valve leaflets with the left ventricular wall, and prevent the valves from collapsing into the left atrium. The equator is the short-axis plane where the left ventricular diameter is largest. Along the circumference the left ventricle is usually divided into four approximately equally sized regions: the anterior, septal, posterior, and lateral (or free) walls. The septum is the wall that separates the left from the right ventricle. The inner and outer surfaces of the left ventricular wall are called the endocardium and the epicardium, respectively. The 


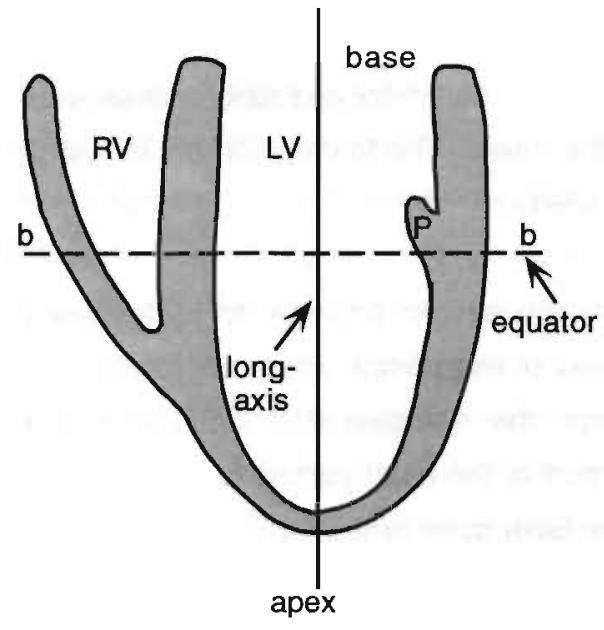

Section a-a

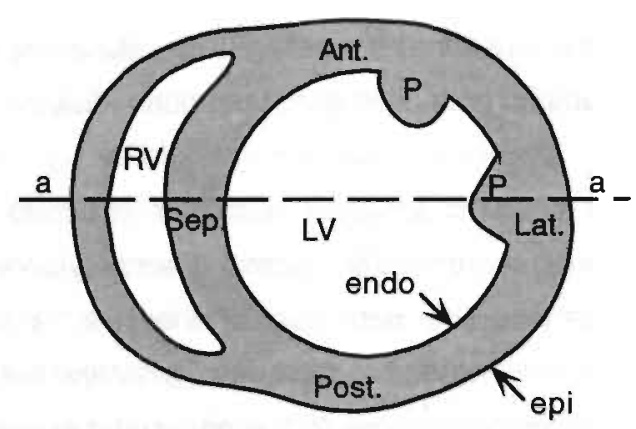

Section b-b

Figure 1.1 Schematic representation of the cardiac left ( $L V)$ and right (RV) ventricles, including the papillary muscles $(P)$. Section a-a gives a long-axis cross-section, while section $b-b$ gives a short-axis cross-section, as viewed from the apex. The equator is the region where the left ventricle is at its widest. The base and apex are the upper and lower parts of the left ventricle, respectively. Endocardium (endo) and epicardium (epi) indicate the inner and outer parts of the wall. The short-axis cross-section is subdivided into four quadrants: the anterior (Ant.), lateral (Lat.), posterior (Post.), and septal (Sep.) walls.

muscular tissue in between the endo- and the epicardium is the myocardium proper. Subendocardium, mid-wall, and subepicardium indicate three regions from the inner to the outer wall surface. Directions relative to the left ventricular geometry are indicated by the terms circumferential, radial, and longitudinal. The circumferential and the radial (or transmural) directions are defined as the directions parallel and perpendicular to the left ventricular wall in a short-axis cross-section, respectively. The longitudinal direction is the direction perpendicular to the short-axis plane.

Coronary arteries, originating from the aorta, supply the heart wall with blood. The larger coronary arteries are located on the outer surface of the heart, while smaller arterial side branches penetrate nearly perpendicularly into the wall. The left coronary 
artery supplies primarily the anterior and lateral portions of the left ventricle, whereas the right coronary artery supplies most of the right ventricle as well as the posterior part of the left ventricle (Guyton, 1991).

In the present thesis attention is paid to myocardial infarction. Myocardial infarction occurs due to inadequate blood supply (ischemia) of certain duration. Myocardial ischemia is often caused by atherosclerosis of the coronary arteries. In this process, atherosclerotic plaques develop on the vessel wall. Thrombi, which are blood clots formed on the plaque, may protrude into the vessel lumen, thus blocking coronary artery blood flow partially or even entirely. Emboli, which are clots that break away from the atherosclerotic plaque, may also lead to acute coronary artery occlusion more distally along the vessel. Even short and mild ischemia hampers the contraction of the involved muscle fibers, but the underlying cardiac tissue may survive if myocardial blood flow is restored in time. If coronary blood flow is severely diminished or blocked, the supplied cardiac tissue dies, resulting in myocardial infarction. Degeneration of the dead muscle fibers causes thinning of the cardiac walls in these regions.

\subsection{DEFORMATION OF THE CARDIAC WALL}

\subsubsection{Measures of cardiac wall deformation}

Deformation of the cardiac wall can be described on a global or on a smaller, local scale. Examples of global wall deformation are torsion of the left ventricie around its long axis, or changes in the endocardial circumference. Local wall deformation is often characterized by strain (McCulloch et al., 1989). Given a coordinate system, normal strains indicate the relative extension of line segments along the coordinate directions. Shear strains indicate the extent to which the angle between two of such perpendicular line segments changes during the deformation. Principal strains are the strains along the principal directions. Along these directions there is no shear strain. The principal strains are the maximum and minimum values of strain, regardless the choice of the coordinate system used. 


\subsubsection{Techniques to quantify cardiac wall deformation}

Over the years many techniques have been developed to quantify cardiac wall deformation, ranging from invasive techniques performed in experiments on animals to noninvasive techniques used routinely in the clinic.

Invasive techniques to quantify cardiac wall deformation include ultrasound in combination with the use of implanted ultrasonic crystals (Gallagher et al., 1982), electromagnetic inductive methods using coils attached to the epicardium (Arts et al., 1982), video-analysis of optical markers attached to the epicardium (Prinzen et al., 1986), and cine-radiography of implanted lead beads (Waldman et al., 1985; Ingels et al., 1989). Using these techniques, a great deal of knowledge was obtained concerning local (3D) cardiac wall deformation. However, application of the techniques in patients is limited due to their invasive nature.

Noninvasive imaging techniques that are currently used routinely in the clinic to measure cardiac wall deformation are echocardiography (Levine et al., 1986) and cine MRI (Van der Wall et al., 1997). These methods are limited in that they assess primarily the motion of the heart contours, i.e., the endocardium and the epicardium. Motion lateral to the wall cannot be easily determined because image contrast within the wall is generally insufficient.

In this respect MR cardiac tagging (Zerhouni et al., 1988; Axel and Dougherty, 1989; McVeigh, 1996) is promising. Using this MRI technique contrast is obtained by tags, which are artificially created by locally altering the magnetization of the cardiac tissue. This allows for quantitative analysis of $3 D$ cardiac wall deformation. The time over which tags can be followed is limited to $200-700 \mathrm{~ms}$ due to (T1) magnetic relaxation. The MR cardiac tagging technique is still in an experimental phase due to the limited availability of user-friendly methods to analyze the images.

Another method to be mentioned is the MRI velocity-encoding technique (McVeigh, 1996), which is also still in an experimental phase. In this case a (phase) difference technique is used to obtain direct estimates of $3 \mathrm{D}$ cardiac wall motion. An overview of the velocity-encoding technique as compared to cardiac tagging is given by McVeigh (McVeigh, 1996). Generally, the velocity-encoding technique does not suffer from 
magnetic relaxation effects, so that wall motion can be quantified during the entire cardiac cycle. The technique is, however, more susceptible to motion artifacts than MR cardiac tagging.

\subsubsection{Deformation of the healthy left ventricle during systole}

Basic global modes of deformation of the healthy left ventricle are volume ejection, torsion, and short-axis and long-axis shortening (Arts et al., 1982; Arts et al., 1992). During systole, volume-ejection and torsion were found to be important, besides rigid body motion. Volume-ejection is related to the overall contraction of the left ventricle such that blood is ejected into the aorta. During systole, generally about $50-70 \%$ of the blood in the cavity is ejected. Torsion is the twisting motion around the left ventricular long-axis, i.e., rotation of the apex with respect to the base, and can be represented by the circumferential-longitudinal shear angle (Arts et al., 1982). Generally, systolic torsion is about 5-8 degrees. Torsion reflects the interplay between endocardial and epicardial muscle fibers, causing homogenization of fiber strains across the wall (Arts et al., 1982; Arts et al., 1984). In the healthy canine left ventricle, a relation was found (Arts et al., 1984) between systolic torsion and volume ejection that is relatively independent of preload (end-diastolic left ventricular cavity volume) and afterload (end-diastolic aortic pressure). It should be noted that Magnetic Resonance cardiac tagging is a very suitable tool to measure torsion, in contrast to more conventional techniques such as echocardiography.

In addition to the above-described basic modes of wall deformation there are local differences in normal systolic deformation along the circumference (Buchalter et al., 1990), from apex to base (Young et al., 1994), and across the wall (Waldman et al., 1985). Generally, strains are larger in the endocardium than in the epicardium. Circumferential strains, for instance, are about $40 \%$ in the subendocardial layers and only about $20 \%$ in the subepicardial ones (Clark et al., 1991). Strain along the fiber direction, however, is relatively homogeneous throughout the wall during systole (Delhaas et al., 1993; Rademakers et al., 1994). This homogeneity may be attributed to torsional motion of the heart, having a complex muscle fiber architecture (Arts et al., 1979; Rijcken et al., 1997). In the various regions of the left ventricle also temporal differences in wall deformation are reported (Naito et al., 1996). 
Furthermore, the above-described deformations are often sensitive to alterations in preload, afterload, and contractile state of the left ventricle (Hansen et al., 1991).

\subsubsection{Pathologies and their influences on left ventricular deformation}

Ventricular deformation is very sensitive to disorders such as myocardial infarction or ischemia, asynchronous electrical activation, and hypertrophic cardiomyopathy. Because in the present thesis attention is paid to myocardial infarction, in this section its effects on the left ventricular deformation pattern are briefly discussed. Aberrant wall deformation related to other pathologies is discussed elsewhere (Mirsky et al., 1983; Waldman and Covell, 1987; Bouton et al., 1991; Maier et al., 1992; Pawlush et al., 1993; Young et al., 1994).

Myocardial ischemia and infarction were shown to influence left ventricular wall deformation in a variety of studies (Prinzen et al., 1984; Prinzen et al., 1989; Kramer et al., 1993; Buchalter et al., 1994; Azhari et al., 1995; Lima et al., 1995; Rechavia et al., 1995; Azhari et al., 1996; Bovendeerd et al., 1996; Kramer et al., 1996; Konermann et al., 1997; Kramer et al., 1997; Kramer et al., 1997; Marcus et al., 1997; Van der Wall et al., 1997). The precise influence depends on the location and severity of the infarction (Buchalter et al., 1994), but the following features prevail. Already within 10-20 seconds after onset of occlusion of a coronary artery, wall deformation during the contraction phase of the left ventricle is altered (Prinzen et al., 1984). Furthermore, due to the fact that muscle fibers are mutually connected, the influences of an infarction are not confined to the infarcted tissue alone, but noticeable in a relatively large part of the left ventricle (Prinzen et al., 1989; Rechavia et al., 1995; Kramer et al., 1996; Marcus et al., 1997). In the infarcted region the wall thins during systole, contrary to the occurrence of wall thickening in the remote healthy regions. Because in infarcted regions the wall is often already thinner than in the healthy regions, the thinning during systole may be very pronounced. In the infarcted region, circumferential shortening is reduced, or even stretch may occur. In the non-infarcted, remote regions often a hyperfunction can be observed (Marcus et al., 1997).

The influences of myocardial infarction on left ventricular deformation patterns change with time, over periods of days or months. After myocardial infarction, remodeling of the left ventricular wall takes place (Kramer et al., 1993; Konermann et 
al., 1997; Kramer et al., 1997). This means that the left ventricle adapts to the new situation in order to compensate for the loss of functional muscular tissue. Kramer et al (Kramer et al., 1993) showed that regional differences in circumferential shortening were greatest in the first week after myocardial infarction, decreased partly between 1 and 8 weeks, but remained detectable up to 6 months after myocardial infarction.

\subsection{BASIC PRINCIPLES OF MAGNETIC RESONANCE IMAGING AND CARDIAC TAGGING}

\subsubsection{Magnetic Resonance Imaging}

This section is confined to a brief description of the aspects of Magnetic Resonance Imaging (MRI) and cardiac tagging that are relevant for a general understanding of the presented work. For more detailed information on the physical and technical principles of MRI the reader is referred to textbooks (Philips, 1996; Vlaardingerbroek and den Boer, 1996).

MRI is an imaging technique that uses the magnetic properties of the atomic nuclei of the materials or tissues to be imaged. In most clinical applications, including cardiac tagging, the nuclei of hydrogen atoms $\left(\mathrm{H}_{1}^{1}\right)$, i.e. protons, are employed. Protons have a magnetic moment, or spin, that can be interpreted as the proton behaving like a small magnet (Feynman et al., 1963). The large number of proton spins within a given amount of tissue add up to a macroscopic magnetic moment, or magnetization. This magnetization is characterized by a magnitude and a direction. When no external magnetic field is present the individual proton spins are oriented randomly, so that they cancel each other out, making the magnetization equal to zero. Under the influence of a large external magnetic field, such as the permanent magnetic field within the core of an MRI scanner, however, the proton spins add up to a net magnetization aligned with the magnetic field. This parallel magnetization is called longitudinal magnetization.

One of the key features in MRI is to excite the protons in the tissue, using radiofrequency (RF) electromagnetic pulses, thus flipping the longitudinal magnetization towards the transverse plane. As a result, the magnetization starts to precess around the direction of the magnetic field (figure 1.2), similar to the 


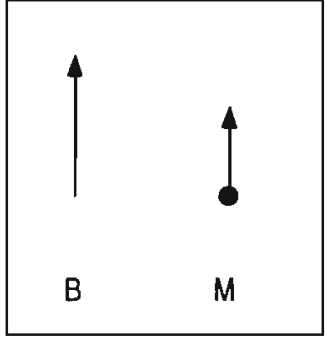

a

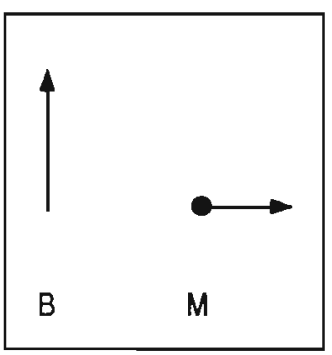

b

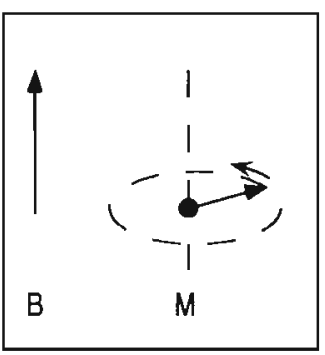

C

Figure 1.2 a. Magnetization $(M)$ is aligned along magnetic field $(B) ; b$. Magnetization is flipped over to the transverse plane; c. Magnetization precesses around direction of magnetic field.

precession of a spinning top under the influence of gravity. The frequency of this precession, the so-called Larmor frequency, is proportional to the magnetic field strength $\left(42.48 \mathrm{MHz} / \mathrm{T}\right.$ for $\left.\mathrm{H}_{1}^{1}\right)$. After excitation, the magnetization starts to realign with the magnetic field due to interactions between the spins and their surroundings. The recovery of longitudinal magnetization, called longitudinal relaxation, occurs exponentially, with a characteristic time T1. For most biological tissues $\mathrm{T} 1$ is on the order of 200-1000 ms at a magnetic field strength of 1 Tesla. Simultaneously, the spins, which precess coherently immediately after excitation, start to get out of phase due to interactions between the individual spins, reducing the transverse magnetization. This transverse relaxation also occurs exponentially, now with a characteristic time T2, which for most biological tissues is on the order of $40-100 \mathrm{~ms}$, independent of the magnetic field strength. If the applied magnetic field is inhomogeneous, the effective transverse relaxation occurs even faster, with a characteristic time $\mathrm{T}^{*}$, typically on the order of $20 \mathrm{~ms}$.

During the time the transverse magnetization precesses around the magnetic field direction, a current, alternating at the Larmor frequency, is induced in surrounding magnetic receiver coils. By varying the magnetic field strength as a function of position, tissues at different locations induce different frequencies. Analysis of the frequency contents of the (demodulated) received signals reveals information on the location of the spinning atomic nuclei. The gradients in the magnetic field strength are employed by a separate set of gradient coils within the MRI scanner. In practice, one 
gradient is applied during excitation of the spins, in order to select an image slice, while two other gradients are applied subsequently to obtain spatial resolution within the image plane.

After the RF excitation pulse is given the signals induced in the receiver coils decay exponentially with a characteristic time $\mathrm{T}^{*}$, due to dephasing of the proton spins. Immediately after the pulse the receiver coils have a dead time in which no signals can be detected. However, dephasing of the proton spins can be reversed partly by an appropriate subsequent RF pulse (spin-echo MRI) or by reversing magnetic field gradients (gradient-echo MRI). Then, after the so-called echo-time (TE), a peak occurs in the signals induced in the receiver coils. The signals during the time period around the echo-time are used to perform the frequency analysis on.

Generally, the time period during which the induced signals are large enough to perform a frequency analysis on is too short to obtain all needed information. Therefore, methods have been developed to gather this information in a number of so-called phase-encoding steps. In each phase-encoding step an RF excitation pulse is given in combination with a different value for the magnetic field gradient in one of the two transverse directions, each time collecting a different part of the frequency spectrum. The time between successive excitations is called the repetition time (TR). In cardiac imaging signal acquisition is performed by triggering on the R-wave in the electrocardiogram for each phase-encoding step, assuming reproducible cardiac motion during the time of the scan.

Contrast between different tissues (e.g. liver, kidney, fat, myocardium, tumor, lung, etc.) in the images can be obtained. Here, the property is used that the T1 and T2 relaxation times are characteristic of the different tissues. By an appropriate choice of TE and TR, images can be made that highlight the differences in T1, T2, or T2* between different tissues.

\subsubsection{Magnetic Resonance cardiac tagging}

MR cardiac tagging is a sophisticated method to mark the myocardium with tags that can be followed in time. MR-tagged images can be made in various ways (Zerhouni et al., 1988; Axel and Dougherty, 1989; McVeigh and Atalar, 1992; Thomsen, 1992; 


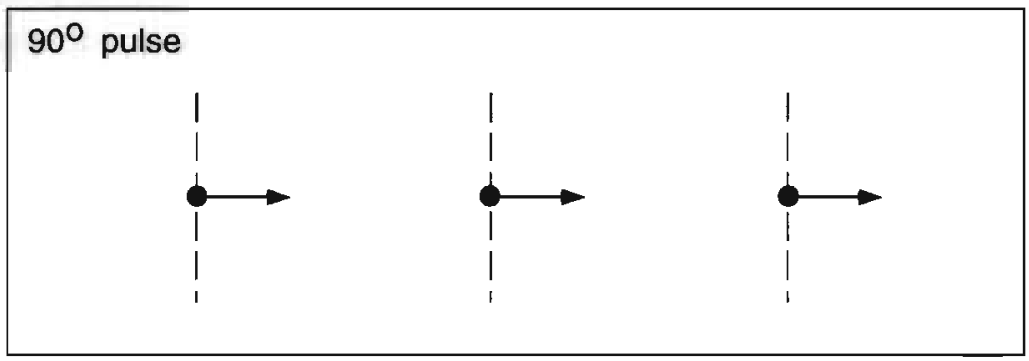

a

precession in transverse plane
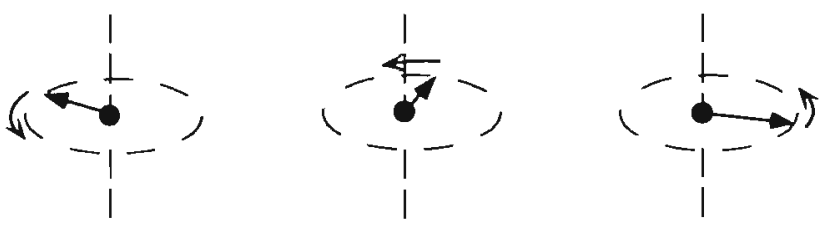

gradient direction

b

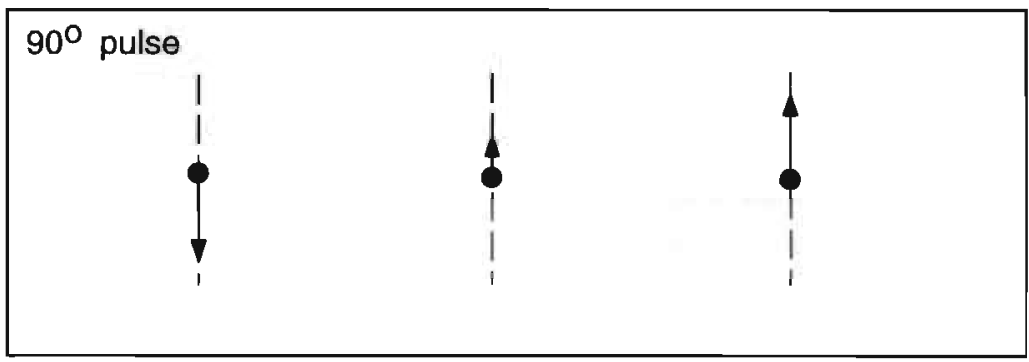

C

Figure 1.3 a. An RF-pulse is given to flip the magnetization (denoted by the arrow) to the transverse plane. $b$. Due to a gradient in the magnetic field, the magnetization precesses with different frequencies along the gradient direction. The phase of precession of the magnetization is therefore modulated along the gradient direction; c. A second RF-pulse is given to flip the magnetization back to the longitudinal direction. Due to the earlier differences in phase, the longitudinal magnetization is now modulated with a spatial frequency that depends on the strength of the magnetic field gradient and the time the gradient was switched on.

Fischer et al., 1993; Tang et al., 1995; McVeigh, 1996). In general, for each phase encoding step a specific tagging sequence is applied prior to the imaging sequence. 
In the studies performed in this thesis, the tagging method called Spatial Modulation of the Magnetization (SPAMM) (Axel and Dougherty, 1989) was used. In its simplest form 2 RF-pulses are employed (figure 1.3). The first RF-pulse flips the magnetization vector to the transverse plane. Immediately thereafter a magnetic field gradient is applied in a direction parallel with the image plane. This causes the frequency of precession of the transverse magnetization to depend linearly on the location along the gradient direction. A few milliseconds later, when the different spins along the gradient direction are out of phase, the gradient is switched off and a second RFpulse is given to flip the transverse magnetization back to a plane parallel with the longitudinal direction. The remaining transverse magnetization decays within $20 \mathrm{~ms}$ due to $\mathrm{T}^{*}$ relaxation. The longitudinal magnetization is now modulated with a spatial frequency depending on the strength of the magnetic field gradient and the time the gradient was switched on. When this tagging sequence is applied prior to the imaging sequence in T1-weighted MR image acquisition, the modulated longitudinal magnetization shows in the images as alternating dark and light stripes, or tags, with a sinusoidal intensity profile. The tagging sequence can also be applied in two mutually perpendicular directions, which results in a grid-like pattern of tags. Acquiring MR signals at a number of different times after application of the tagging sequence, in each phase-encoding step, gives a time series of images in which the tags move along with the tissue they were placed in (figure 1.4). Due to T1-relaxation and the tissue motion itself, the tags fade away within 200 to $700 \mathrm{~ms}$, depending on the tagging and imaging techniques employed (Axel and Dougherty, 1989; McVeigh and Atalar, 1992; Fischer et al., 1993; Tang et al., 1995).

\subsection{METHODS TO ANALYZE MR TAGGED IMAGES}

A number of methods have been developed to determine left ventricular wall motion from MR-tagged images. In this section some of the general principles underlying these methods will be discussed.

Many of the recently developed methods acquire MR-tagged images from multiple cross-sections through the left ventricle. In general, the analysis methods are based on the strategy to first determine 2D motion within the individual image planes and then to combine the motion information of mutually perpendicular image planes to quantify 3D wall motion (Young and Axel, 1992; Azhari et al., 1993; Denney and 


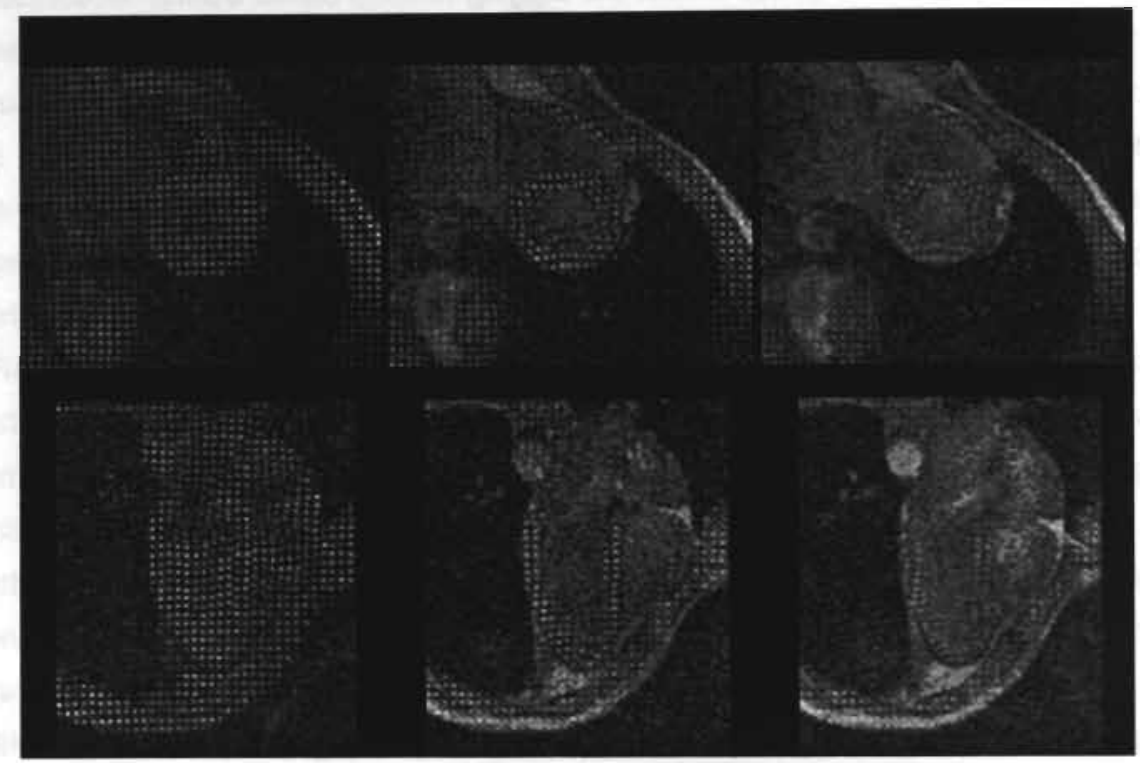

Figure 1.4 MR-tagged short-axis (above) and long-axis (below) images of the contracting cardiac left ventricle. The images were acquired 20 (left), 140 (middle), and $240 \mathrm{~ms}$ (right) after the R-wave of the ECG. The motion of the tags corresponds to myocardial deformation. Due to T1-relaxation the tags slowly fade away, until after about $250 \mathrm{~ms}$ noise becomes dominant. In the left ventricular cavity the tags disappear shortly after application due to the ejection of blood.

Prince, 1995; O'Dell et al., 1995; Young et al., 1995; Moulton et al., 1996; Park et al., 1996; Denney and McVeigh, 1997).

The quantification of $2 \mathrm{D}$ motion within an image plane is generally achieved by following the in-plane motion of the individual tags. To this purpose, several methods have been developed to detect tags. The most widely used approaches are based either on the matching of tag profile templates (McVeigh and Zerhouni, 1991; Guttman et al., 1994; Kraitchman et al., 1995) or the use of so-called snakes (Kass et al., 1988; Kumar and Goldgof, 1994; Young et al., 1995; Park et al., 1996), which are splines that are attracted to tag lines. With these approaches in-plane tag motion can be determined highly accurately. However, generally a great deal of user-interaction is required. This is mainly caused by the need to delineate left ventricular wall 
contours in all separate images, i.e., in all cross-sections and at all time instants. Despite the large amount of effort that has been expended on this problem, at the present state of the art it is not possible to delineate left ventricular wall contours fully automatically, and manual corrections are still needed (Guttman et al., 1994; Axel et al., 1995). After delineation of wall contours, tags can be detected by means of above-mentioned methods in a highly, but not completely automated way. Another problem encountered in the tag detection methods is that they often have to be fine tailored to the specific images (tag shape, image quality/contrast) used in order to ensure accurate tracking of the tags.

An approach to quantify in-plane motion that does not depend on the detection of individual tags is the variable brightness optical flow method (Prince and McVeigh, 1992). Although no separate tags need to be detected, a number of image properties, such as the variable brightness and noise in the images, must be known a priori, which is a limitation of this approach. Additionally, errors in motion estimates accumulate when sequences of images are tracked.

Most of the methods mentioned before were primarily used to quantify local wall deformation. This proved to be a fruitful approach when studying the mechanics of the left ventricular wall locally, for instance, to distinguish healthy from diseased tissue (Young et al., 1994; Azhari et al., 1995; Marcus et al., 1997). Local deformation parameters, such as circumferential or principal strains, can be displayed by pseudocolors overlaid on the original images or on a reconstructed 3D representation of the left ventricular geometry (Axel et al., 1995; McVeigh, 1996). A dynamic display of the time evolution of the local deformation parameters enables the clinician to qualitatively evaluate motion disorders. Quantitative interpretation of the motion information, however, is still problematic. Complex post-processing by a skilled operator seems to be necessary, making this approach less suitable as a quantitative clinical tool. Often, local motion estimates have to be averaged over entire regions of the left ventricular wall. An alternative approach is to describe wall motion directly on a more global scale in terms of a limited number of relevant motion parameters (Park et al., 1996; Park et al., 1996). 


\subsection{OBJECTIVES OF THE THESIS}

\subsubsection{Objectives}

The possibility to determine $3 D$ cardiac wall deformation in great detail in a completely noninvasive way makes MR cardiac tagging a potentially useful extension of the current cardiac diagnostic tools. However, the need for large amounts of userinteraction to analyze the images and the need for complex post-processing have prevented a thorough clinical assessment of the method. The general objective of the studies presented in this thesis was to develop a method to quantify left ventricular motion from MR-tagged images that is automated to a high degree and is flexible in regard to the specific tagging technique used. The methods to be developed should furthermore be flexible regarding the desired spatial resolution of the motion field, which is associated with the number and locations of the cross-sections through the left ventricle. In addition, it was our objective to pay attention to present the motion information in such a way that it is easily interpretable for a physician. To accomplish this, the strategy is followed to incorporate foreknowledge about wall motion of the healthy or pathological left ventricle into the motion detection techniques. This foreknowledge is represented in a kinematic model, which describes wall motion by a number of characteristic motion patterns. Examining a limited number of characteristic motion pattems may facilitate the interpretation of the results.

\subsubsection{Description of wall deformation by means of a kinematic model}

In a kinematic model of the left ventricular wall regional wall displacement is characterized by a set of kinematic parameters, varying as a function of time. Each parameter represents a different mode of motion. Parameters can be more or less global or local. A global parameter describes a mode of motion that extends over a relatively large part of the wall, while local parameters are confined to the description of the motion of relatively small regions. Thus, global parameters, such as torsion or volume ejection, can give insight into overall left ventricular function, and local parameters, such as local strains, yield information about local cardiac mechanics. In the analysis there is essentially no difference between global and local parameters; there is only a difference in scale. Basically, a kinematic model is an interpolation method, i.e., a method to obtain information about the motion of an arbitrary point in 
the wall starting from a limited number of kinematic parameter values (e.g. motion of a number of points). Another aspect of kinematic models is that foreknowledge can be incorporated into the description of wall motion. In case of specific cardiac pathologies, wall motion abnormalities can be described by means of a characteristic motion pattem. Thus, sensitivity in detecting the motion disorder may be enhanced.

\subsubsection{Outline of the contents of the thesis}

In order to get more acquainted with the problems associated with the quantification of left ventricular wall motion from MR-tagged images, the strategy is followed to first develop a method to automatically detect tags and determine $2 \mathrm{D}$ in-plane tag motion (chapters 2 and 3 ). In the later studies (chapters 4 and 5), methods are developed to determine 3D wall motion by simultaneously analyzing MR-tagged images from multiple cross-sections, without the need to detect individual tags.

In chapter 2 MR-tagged images of one short-axis cross-section, acquired in healthy volunteers and in patients with myocardial infarction, are analyzed. A method is developed to detect tags in MR-tagged images automatically. To this purpose, techniques are adapted which have been successfully employed in analysis of video image analysis of epicardial deformation (Prinzen et al., 1986). Thus found tag tracks are fitted to a kinematic model of cross-sectional motion. This kinematic model describes the basic modes of cross-sectional motion, supplemented by a simple description of the influence of a regional dysfunction.

In chapter $\mathbf{3}$ the method is extended in order to determine torsion of the left ventricle. MR-tagged images from two parallel short-axis cross-sections are acquired in healthy volunteers. Tags are detected and followed for each cross-section independently, as described in chapter 2. Motion information from the two cross-sections is combined in order to quantify torsion and contraction (cross-sectional cavity area change) of the left ventricle. Furthermore, a prediction regarding the relation between torsion and contraction of the left ventricle during the ejection phase, made by a mathematical model of left ventricular mechanics, is verified.

In chapter 4 the step is made towards the use of a kinematic model for full 3D analysis of multiple tagged images without the need to detect individual tags. MR- 
tagged images are acquired from two short-axis cross-sections and two perpendicular long-axis cross-sections in healthy volunteers. A previously developed 3D kinematic model is used to describe the normal global pattern of left ventricular wall motion. Parameters in the kinematic model are determined from the images without the need for tag detection, making the method highly automated and to a great extent independent of the tagging technique used.

In chapter 5 a new kinematic model is developed, which allows for a description of regional abnormalities. Again, MR-tagged images are acquired from two short-axis cross-sections and two perpendicular long-axis cross-sections, now in healthy volunteers as well as in patients with myocardial infarction. The parameters in the kinematic model are estimated in the same way as in chapter 4 . The method is applied to locate the site of the myocardial infarction in the patients.

The thesis is concluded with a discussion of the findings in chapter 6 .

\section{REFERENCES}

Arts, T., Hunter, W. C., Douglas, A., Muytjens, A. M. M., and Reneman, R. S. (1992) Description of the Deformation of the Left Ventricle by a Kinematic Model. $J$ Biomechanics 25, 1119-1127.

Arts, T., Meerbaum, S., Reneman, R. S., and Corday, E. (1984) Torsion of the Left Ventricle during the Ejection Phase in the Intact Dog. Cardiovasc Res 18, 183-193.

Arts, T., Reneman, R. S., and Veenstra, P. C. (1979) A model of the mechanics of the left ventricle. Ann Biomed Eng 7, 299-318.

Arts, T., Veenstra, P. C., and Reneman, A. S. (1982) Epicardial Deformation and Left Ventricular Wall Mechanics during Ejection in the Dog. Am J Physiol 243, H379-H39O.

Axel, L., Bloomgarden, D. C., Chang, C.-N., Fayad, Z. A., Kraitchman, D. L., and Young, A. A. (1995) An integrated program for 2-D and 3-D analysis of heart wall motion from Magnetic Resonance Imaging. In Computers in Cardiology. Vienna: IEEE.

Axel, L. and Dougherty, L. (1989) Heart wall motion: improved method of spatial modulation of magnetization for MR imaging. Radiology 172, 349-350.

Axel, L. and Dougherty, L. (1989) MR Imaging of Motion with Spatial Modulation of the Magnetization. Radiology 171, 841-845.

Azhari, H., Oliker, S., Rogers, W. J., Weiss, J. L., and Shapiro, E. P. (1996) Threedimensional mapping of acute ischemic regions using artificial neural networks and tagged MRI. IEEE T Biomed Eng 43, 619626.

Azhari, H., Weiss, J. L., Rogers, W. J., Siu, C. O., and Shapiro, E. P. (1995) A noninvasive comparative study of 
myocardial strains in ischemic canine heart using tagged MRI in 3-D. Am J Physiol 268, H1918-H1926.

Azhari, H., Weiss, J. L., Rogers, W. J., Siu, C. O., Zemouni, E. A., and Shapiro, E. P. (1993) Noninvasive Quantification of Principal Strains in Normal Canine Hearts using Tagged MRl images in 3-D. Am J Physiol 264, H205-H216.

Bouton, S., Yang, A., McCrindle, B. W., Kidd, L., McVeigh, E. R., and Zerhouni, E. A. (1991) Differentiation of tumor from viable myocardium using cardiac tagging with MR Imaging. JCAT 15, 676-678.

Bovendeerd, P. H. M., Arts, T., Delhaas, T., Huyghe, J. M., Van Campen, D. H., and Reneman, R. S. (1996) Regional wall mechanics in the ischemic left ventricle: numerical modeling and dog experiments. Am J Physiol 270, H398-H410.

Buchalter, M. B., Rademakers, F. E., Weiss, J. L., Rogers, W. J., Weisfeldt, M. L., and Shapiro, E. P. (1994) Rotational deformation of the canine left ventricle measured by magnetic resonance tagging: effects of catecholamines, ischaemia, and pacing. Cardiovasc Res 28, 629-635.

Buchalter, M. B., Weiss, J. L., Rogers, W. J., Zerhouni, E. A., Weisfeldt, M. L., Beyar, R., and Shapiro, E. P. (1990) Noninvasive Quantification of Left Ventricular Rotational Deformation in Normal Humans Using Magnetic Resonance Imaging Myocardial Tagging. Circulation 81, 1236-1244.

Clark, N. R., Reichek, N., Bergey, P., Hoftman, E. A., Brownson, D., Palmon, L., and Axel, L. (1991) Circumferential Myocardial Shortening in the Normal Human Left Ventricle - Assessment by Magnetic Resonance Imaging Using Spatial Modulation of Magnetization. Circulation 84, 67-74.
Delhaas, T., Arts.T., Bovendeerd, P. H. M., Prinzen, F. W., and Reneman, R. S. (1993) Subepicardial fiber strain and stress as related to left ventricular pressure and volume. Am J Physiol 264, H1548-H1559.

Denney, T. S. and McVeigh, E. R. (1997) Model-free reconstruction of threedimensional myocardial strain from planar tagged MR images. JMR/7, 799-810.

Denney, T. S. and Prince, J. L. (1995) Reconstruction of $3-D$ left ventricular motion from planar tagged cardiac MR images: an estimation theoretic approach. IEEE T Med Imaging 14, 625-635.

Feynman, R. P., Leighton, R. B., and Sands, M. (1963) The Feynman lectures on physics. Addison-Wesley Publishing Company, Reading, Mass.

Fischer, S. E., McKinnon, G. C., Maier, S. E., and Boesiger, P. (1993) Improved Myocardial Tagging Contrast. Magn Reson Med 30, 191-200.

Gallagher, K. P., Osakada, G., Hess, O. M., Koziol, J. A., Kemper, W. S., and Ross Jr., J. (1982) Subepicardial segmental function during coronary stenosis and the role of myocardial fiber orientation. Circ Res 50, 352-359.

Guttman, M. A., Prince, J. L., and McVeigh, E. R. (1994) Tag and contour detection in tagged MR images of the left ventricle. IEEE T Med Imaging 13, 74-88.

Guyton, A. C. (1991) Textbook of medical physiology. W.B. Saunders Company, Philadelphia.

Hansen, D. E., Daughters, G. T., Alderman, E. L., Ingels, N. B., Stinson, E. B., and Miller, D. C. (1991) Effect of volume loading, pressure loading, and inotropic stimulation on left ventricular torsion in humans. Circulation 83, 1315-1326. 
Ingels, N. B., Hansen, D. E., Daughters, G. T., Stinson, E. B., Alderman, E. L., and Miller, D. C. (1989) Relation between longitudinal, circumferential, and oblique shortening and torsional deformation in the left ventricle of the transplanted human heart. Circ Res 64, 915-927.

Kass, M., Witkin, A., and Terzopoulos, D. (1988) Snakes: Active Contour Models. Int $\checkmark$ Comp Vision 1, 321-331.

Konermann, M., Sanner, B. M., Horstmann, E., Grotz, J., Laschewski, F., Josephs, W., Odenthal, H. J., and Sturm, A. (1997) Changes of the left ventricle after myocardial infarction - estimation with cine Magnetic Resonance Imaging during the first six months. Clin Cardiol 20, 201-212.

Kraitchman, D. L., Young, A. A., Chang, C.-N., and Axel, L. (1995) Semi-automatic tracking of myocardial motion in MR-tagged images. IEEE T Med Imaging 14, 422-433.

Kramer, C. K., Rogers, W. J., Theobald, T. M., Power, T. P., Geskin, G., and Reichek, N. (1997) Dissociation between changes in intramyocardial function and left ventricular volumes in the eight weeks after first anterior myocardial infarction. JACC 30 , 1625-1632.

Kramer, C. M., Lima, J. A. C., Reichek, N., Ferrari, V. A., Llaneras, M. R., Palmon, L. C., Yeh, I.-T., Tallant, B., and Axel, L. (1993) Regional differences in function within noninfarct myocardium during left ventricular remodeling. Circulation 88, 1279-1288.

Kramer, C. M., Rogers, W. J., Geskin, G., Power, T. P., Theobald, T. M., Hu, Y. L., and Reichek, N. (1997) Usefulness of Magnetic Resonance Imaging early after acute myocardial infarction. Am J Cardiol 80, 690-695.
Kramer, C. M., Rogers, W. J., Theobald, T. M., Power, T. P., Petruolo, S., and Reichek, N. (1996) Remote noninfarcted region dysfunction soon after first anterior myocardial infarction. A Magnetic Resonance Tagging study. Circulation 94, 660-666.

Kumar, S. and Goldgof, D. (1994) Automatic tracking of SPAMM grid and the estimation of deformation parameters from cardiac MR images. IEEE T Med Imaging 13, 122132.

Levine, R. A., Gillam, L. D., and Weyman, A. E. (1986) Echocardiography in cardiac research. In The heart and cardiovascular system. (Edited by Fozzard et al.) Raven Press, New York, 369-542.

Lima, J. A. C., Ferrari, V. A., Reichek, N., Kramer, C. M., Palmon, L., Llaneras, M. R., Tallant, B., Young, A. A., and Axel, L. (1995) Segmental motion and deformation of transmurally infarcted myocardium in acute postinfarct period. Am J Physiol 268, H1304-H1312.

Maier, S. E., Fischer, S. E., McKinnon, G. C., Hess, O. M., Krayenbuehl, H. P., and Boesiger, P. (1992) Evaluation of Left Ventricular Segmental Wall Motion in Hypertrophic Cardiomyopathy with Myocardial Tagging. Circulation 86, 19191928.

Marcus, J. T., Gotte, J. W., Van Rossum, A. C., Kuijer, J. P. A., Heethaar, R. M., Axel, L., and Visser, C. A. (1997) Myocardial function in infarcted and remote regions early after infarction in man: Assessment by Magnetic Resonance tagging and strain analysis. MRM 38, 803-810.

McCulloch, A. D., Smaill, B. C., and Hunter, P. J. (1989) Regional left ventricular epicardial deformation in the passive dog heart. Circ Res 64, 721-733. 
McVeigh, E. R. (1996) MRI of myocardial function: motion tracking techniques. Magn Reson Imaging 14, 137-150.

McVeigh, E. R. and Atalar, E. (1992) Cardiac Tagging with Breath-Hold Cine MRI. Magn Res Med 28, 318-327.

McVeigh, E. R. and Zerhouni, E. A. (1991) Noninvasive measurement of transmural gradients in myocardial strain with MR imaging. Radiology 180, 677-683.

Mirsky, I., Pfeffer, J. M., Pfeffer, M. A., and Braunwald, E. (1983) The Contractile State as the Major Determinant in the Evolution of Left Ventricular Dysfunction in the Spontaneously Hypertensive Rat. Circ Res 53, 767-778.

Moulton, M. J., Creswell, L. L., Downing, S. W., Actis, R. L., Szabo, B. A., Vannier, M. W., and Pasque, M. K. (1996) Spline surface interpolation for calculating 3-D ventricular strains from MRI tissue tagging. Am J Physiol 270, H281-H297.

Naito, H., Arisawa, J., Harada, K., Yamagami, H., Kozuka, T., and Tamura, S. (1996) Normal nonuniformity of left ventricular contraction. Acta Radiologica 37, 767-774.

O'Dell, W. G., Moore, C. C., Hunter, W. C., Zerhouni, E. A., and McVeigh, E. R. (1995) Three-dimensional myocardial deformations: calculation with displacement field fitting to tagged MR images. Radiology 195, 829-835.

Park, J., Metaxas, D., and Axel, L. (1996) Analysis of left ventricular wall motion based on volumetric deformable models and MRI-SPAMM. Med Image Anal 1, 5371.

Park, J., Metaxas, D., Young, A. A., and Axel, L. (1996) Deformable models with parameter functions for cardiac motion analysis from tagged MRI data. IEEE $T$ Med Imaging 15, 278-289.

Pawiush, D. G., Moore, R. L., Musch, T. I., and Davidson, W. R. (1993) Echocardiographic Evaluation of Size, Function and Mass of Normal and Hypertrophied Rat Ventricles. $J$ Appl Physiol 74, 2598-2605.

Philips (1996) Basic principles of MR imaging. Philips Medical Systems, Best, The Netherlands.

Prince, J. L. and McVeigh, E. F. (1992) Motion estimation from tagged MR image sequences. IEEE T Med Imag 11, 238-249.

Prinzen, F. W., Arts, T., Hoeks, A. P. G., and Reneman, R. S. (1989) Discrepancies between Myocardial Blood Flow and Fiber Shortening in the Ischemic Border Zone as assessed with Video Mapping of Epicardial Deformation. Eur $J$ of Physiology 415, 220229.

Prinzen, F. W., Arts, T., Van der Vusse, G. J., and Reneman, R. S. (1984) Fiber shortening in the inner layers of the left ventricular wall as assessed from epicardial deformation during nomoxia and ischemia. $J$ Biomechanics 17, 801-811.

Prinzen, T. T., Arts, T., Prinzen, F. W., and Reneman, R. S. (1986) Mapping of Epicardial Deformation using a Video Processing Technique. J Biomechanics 19, 263-273.

Rademakers, F. E., Rogers, W. J., Guier, W. H., Hutchins, G. M., Siu, C. O., Weisfeldt, M. L., Weiss, J. L., and Shapiro, E. P. (1994) Relation of regional cross-fiber shortening to wall thickening in the intact heart. Circulation 89, 1174-1182.

Rechavia, E., De Silva, R., Nihoyannopoulos, P., Lammertsma, A. A., Jones, T., and Maseri, A. (1995) Hyperdynamic performance of remote myocardium in 
acute infarction - Correlation between regional contractile function and myocardial perfusion. Eur Heart J 16, 1845-1850.

Rijcken, J., Bovendeerd, P. H. M., Schoofs, A. J. G., van Campen, D. H., and Arts, T. (1997) Optimization of cardiac fiber orientation for homogeneous fiber strain at beginning of ejection. $J$ Biomechanics 30 , 1041-1049

Tang, C., McVeigh, E. R., and Zerhouni, E. A. (1995) Multi-shot EPI for improvement of myocardial tagging contrast: comparison with segmented SPGR. Magn Res Med 33, 443-447.

Thomsen, C. (1992) Chess-Board Pattem Spatial Modulation of Magnetization. Acta Radiol 33, 16-23.

Tyberg, J. V., Forrester, J. S., Wyatt, H. L., Goldner, S. J., Parmley, W. W., and Swan, H. J. C. (1974) An analysis of segmental ischomic dysfunction utilizing the pressurelength loop. Circulation 49, 748-754.

Van der Wall, E. E., Van Rugge, F. P., Vliegen, H. W., Reiber, J. H. C., De Roos, A., and Bruschke, A. V. G. (1997) Ischemic heart disease: value of MR techniques. Int $J$ Card Imaging 13, 179-189.

Vlaardingerbroek, M. T. and den Boer, J. A. (1996) Magnetic Resonance Imaging. Springer-Verlag, Berlin.

Waldman, L. K. and Covell, J. W. (1987) Effects of ventricular pacing on finite deformation in canine left ventricles. Am J Physiol 252, H1023-H1030.

Waldman, L. K., Fung, Y. C., and Covell, J. W. (1985) Transmural myocardial deformation in the canine left ventricle. Circ Res 57, 152-163.

Young, A. A. and Axel, L. (1992) Threedimensional motion and Deformation of the Heart Wall: Estimation with Spatial
Modulation of Magnetization - A Modelbased Approach. Radiology 185, 241-247.

Young, A. A., Imai, H., Chang, C. N., and Axel, L. (1994) Two-dimensional Left Ventricular Deformation during Systole using Magnetic Resonance Imaging with Spatial Modulation of Magnetization. Circulation 89, 740-752.

Young, A. A., Kraitchman, D. L., Dougherty, L., and Axel, L. (1995) Tracking and finite element analysis of stripe deformation in magnetic resonance tagging. IEEE $T$ Med Imaging 14, 413-421.

Young, A. A., Kramer, C. M., Ferrari, V. A., Axel, L., and Reichek, N. (1994) Threedimensional left ventricular deformation in hypertrophic cardiomyopathy. Circulation 90, 854-867.

Zerhouni, E. A., Parish, D. M., Rogers, W. J., Yang, A., and Shapiro. E. A. (1988) Human Heart: Tagging with MR Imaging - A Method for Noninvasive Assessment of Myocardial Motion. Radiology 169, 59-63. 


\title{
Chapter 2
}

Kinematic analysis of left ventricular deformation in myocardial infarction using Magnetic Resonance cardiac tagging

\author{
F.W.L. Aelen ${ }^{1}$, T. Arts', D.G.M. Sanders ${ }^{3}$, G.R.P. Thelissen ${ }^{3}$, F.W. Prinzen², \\ R.S. Reneman ${ }^{2}$
}

Departments of Biophysics ${ }^{1}$ and Physiology ${ }^{2}$, Cardiovascular Research Institute Maastricht, Maastricht University, PO Box 616, 6200 MD Maastricht, The Netherlands,

Department of Diagnostic Radiology ${ }^{3}$, University Hospital Maastricht, PO Box 5800, 6202 AZ Maastricht, The Netherlands.

Accepted for publication in: Intemational Joumal of Cardiac Imaging 


\subsection{ABSTRACT}

The Magnetic Resonance (MR) tagging technique provides detailed information about $2 \mathrm{D}$ motion in the plane of observation. Interpretation of this information as a reflection of the 3D motion of the entire cardiac wall is a major problem. In finite element models of the mechanics of the infarcted heart, an infarcted region causes motional asymmetry, extending far beyond the infarct boundary. Here we present a method to quantify such asymmetry in amplitude and orientation. For this purpose images of a short-axis cross-section of the ejecting left ventricle were acquired from 9 healthy volunteers and 5 patients with myocardial infarction. MR-tags were applied in a $5 \mathrm{~mm}$ grid at end-diastole. The tags were tracked by video-image analysis. Tag motion was fitted to a kinematic model of cardiac motion. For the volunteers and the patients the center of the cavity displaced by about the same amount $(p=0.11)$ during the ejection phase: $3.8 \pm 1.4$ and $3.0 \pm 0.9 \mathrm{~mm}$ (mean $\pm s d$ ), respectively. Cross-sectional rotation and the decrease in cross-sectional area of the cavity were both greater in the volunteers than in the patients: $6.4 \pm 1.5$ vs. $3.0 \pm 0.8$ degrees ( $p<0.001$ ), and $945 \pm 71$ vs. $700 \pm 176$ $\mathrm{mm}^{2}(\mathrm{p}=0.02)$, respectively. In the patients, asymmetry of wall motion, as expressed by a sine wave dependency of contraction around the circumference, was significantly enlarged $(p=0.02)$. The proposed method of kinematic analysis can be used to assess cardiac deformation in humans. We expect that by analyzing images of more crosssections simultaneously, the 3D location and the degree of infarction can be assessed efficiently. 


\subsection{INTRODUCTION}

Deformation of the left ventricle is sensitive to cardiac disorders such as myocardial ischemia, cardiac infarction and hypertrophic cardiomyopathy (Prinzen et al., 1989; Maier et al., 1992; Young et al., 1994; Lima et al., 1995). Quantitative assessment of the deformation pattern may therefore be useful in the diagnosis of these diseases. The use of Magnetic Resonance (MR) tagging (Zerhouni et al., 1988; Axel and Dougherty, 1989; Axel et al., 1992; McVeigh, 1996) was a break-through in the kinematic analysis of the heart. Using the technique of Spatial Modulation of the Magnetization (SPAMM) a grid of tags is attached to the cardiac tissue. Assessment of the motion of the grid enables noninvasive quantification of cardiac deformation.

Maier et al (Maier et al., 1992) semi-automatically identified crossing-points of tags and determined rotational and radial displacement. Contours of the left ventricular cross-sections had to be delineated manually in all images. Young et al (Young et al., 1994) analyzed grid line intersection data for displacement, rotation, torsion, and local strains. More recently semi-automatic methods based on active contour models or "snakes" were used to follow deformation of the tagging grid (Kumar and Goldgof, 1994; Kraitchman et al., 1995). These snakes were attracted to salient image features and could be interactively directed by external forces. Supervision and intervention were needed because of image artifacts or indistinct boundaries (Axel et al., 1995).

Interpretation of the MR information as useful clinical knowledge is still a problem. Deformation may be known, but translation of this information to regional function of the myocardial cells is not obvious because of uncertainties in the direction of the myofibers. Another problem is that only a limited amount of image data can be acquired due to the clinically limited acquisition time. The aim of this study was to develop a method to analyze MR tagged images automatically with a focus on deriving the maximum amount of deformation information from a minimum amount of image information.

To describe cardiac deformation we apply a kinematic model. From the viewpoint of data-analysis, the use of such motion foreknowledge enhances the signal to noise ratio in the analysis. The kinematic model is based on finite element calculations of deformation in an infarcted heart (Bovendeerd et al., 1996). From that study, the 
effect of malfunction in the infarcted region appeared to extend far beyond the boundaries of that region. A promising variable for the detection of an infarction was asymmetry of contraction in a short-axis cross-section. This variable may be a more suitable measure for detection of an infarction than wall thickening, which is often used in the clinic (Lessick et al., 1993). With our approach we hope to get a better estimate of location and severity of contractile disorders for a given, limited amount of MR image information.

In the present study we started simple, with just one short-axis slice of MR tagging information. The number of parameters in the kinematic model was minimal, just enough to describe the presence and degree of regional dysfunction. Besides rigid body motion and cavity area ejection, the left ventricular wall was allowed to contract asymmetrically in the first order, i.e., a sine wave dependency of circumferential shortening around the circumference. The sine wave was described by 2 parameters, determining the location and amplitude of asymmetry. User-interaction was limited to the approximate delineation of the region of interest in one image.

\subsection{METHODS}

\subsubsection{Image acquisition}

Magnetic Resonance Images were obtained in 9 healthy volunteers and 5 patients using a 0.5 Tesla MR imaging system (Philips Gyroscan T5-II, Philips Medical Systems, Best, The Netherlands) with body wrap-around coil. The volunteers (age: 25-50 years) had no previous history of cardiac disease. All patients (age: 45-60 years) had an anterior wall infarction, caused by left anterior descending (LAD) coronary artery occlusion, of at least one year old. In all subjects the electrocardiogram (ECG) was used to trigger image acquisition. Prior to the experiment the location and orientation of the left ventricle were determined by compound angulation. For this purpose a transverse image and a coronal-angulated image were acquired sequentially. Adjustments were made so that the cross-section was obtained perpendicular to the left ventricular long axis, about $2 \mathrm{~cm}$ below the attachment of the mitral valve. A series of 16 images (scan) was acquired with time intervals of $19 \mathrm{~ms}$, starting $20 \mathrm{~ms}$ after the $R$ wave in the ECG (figure 2.1). A rectangular grid of tags was attached to the myocardium by spatial modulation of the 


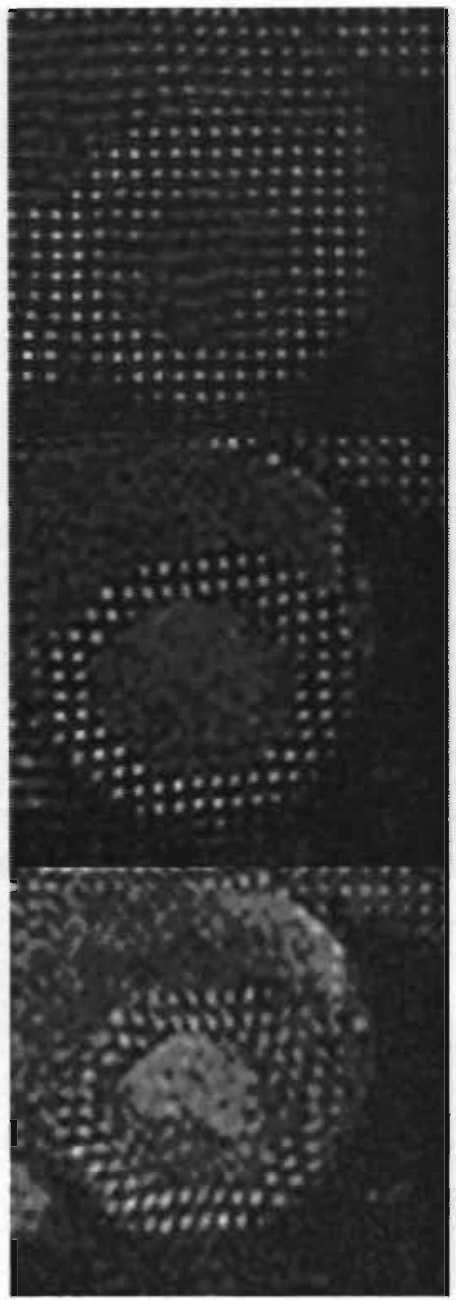

Figure 2.1 MR-tagged short-axis images of the healthy left ventricle during contraction. The images were acquired at 20,96 and $172 \mathrm{~ms}$ (from top to bottom) after the $R$-wave in the ECG. Deformation of the tagging grid is clearly visible. The tags gradually fade away. In the cavity of the left ventricle they disappear shortly after excitation of the grid due to the ejection of blood.

magnetization (SPAMM) immediately before acquisition of the first image. Depending on the visually assessed quality of the last images in this scan, an overlapping second scan was started at 115,134 or 153 ms after the $\mathrm{R}$ wave. The initial inter tag distance was $5 \mathrm{~mm}$ with a tag width of $2.5 \mathrm{~mm}$, in both the horizontal and the vertical direction. 


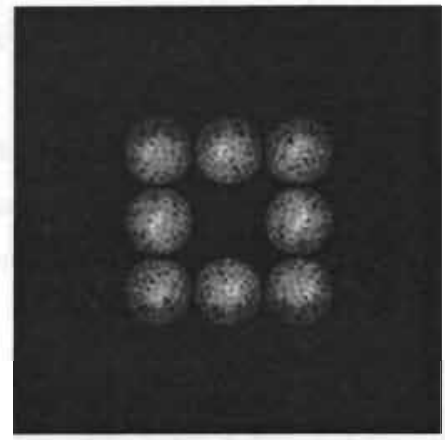

Figure 2.2 Power spectrum after filtering (96 $\mathrm{ms}$ after the R-wave in the ECG). Only frequencies that are considered to be important to the image of the grid are passed through a bandpass filter.

T1-weighted MR imaging was utilized to acquire the short-axis images of the left ventricle. The field of view was $213 \mathrm{~mm}$ with a resolution of $256 \times 256$ pixels. Slice thickness was $8 \mathrm{~mm}$. The repetition time (TR) was equal to the R-R interval, with an echo time (TE) of $10 \mathrm{~ms}$. The tagging grid was generated using two $90^{\circ}$ radiofrequency (RF) pulses for each direction. The RF pulse flip angle for excitation was $40^{\circ}$. Each scan was acquired in about 5-6 minutes.

After image acquisition a $128 \times 128$ pixel region of interest was magnified to $256 \times 256$ pixels by adding zeros to the spatial frequency spectrum.

\subsubsection{Image analysis}

The tags were excited in a rectangular grid pattern. About $200 \mathrm{~ms}$ after excitation (1012 frames), the tags gradualiy faded until finally noise became dominant. To enhance the tag images filtering was performed in the spatial frequency domain. The frequency power spectrum of the grid contains a grid of peaks. Most of the power was concentrated at and near the center of the nine central peaks. The most central peak contains much power, but no grid information. Initially, the other eight peaks were narrow, representing the spatial frequencies of the grid. During contraction, the grid deformed causing the peaks to broaden. Because strains and rotations were limited to about 0.3 and 20 degrees respectively, the width of the peaks also remained 


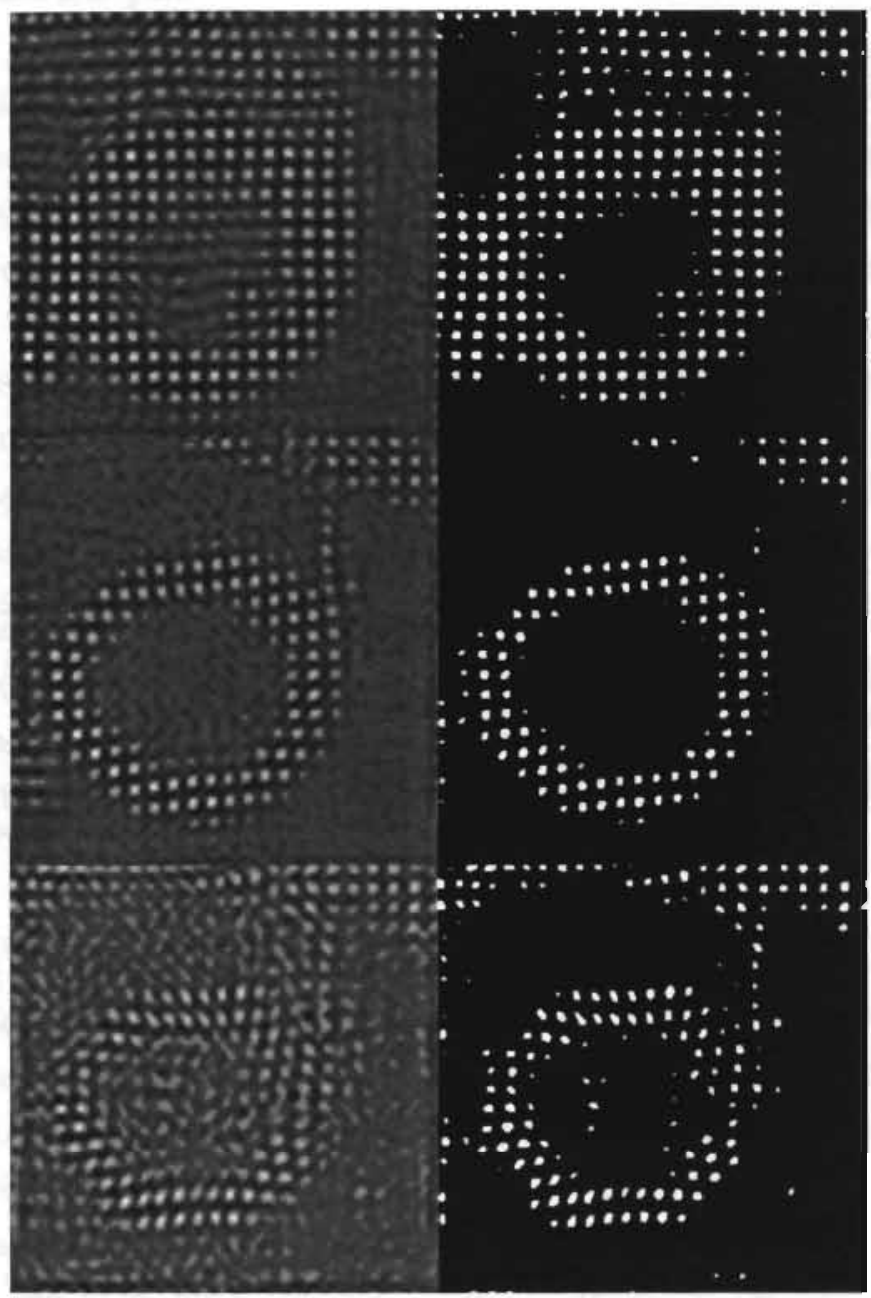

Figure 2.3 A representative series of filtered (left) and thresholded (right) images of a healthy volunteer, 20, 96 and $172 \mathrm{~ms}$ (from top to bottom) after the R-wave in the ECG. Filtering enhanced the detectability of the tags, thus making the images more suitable for thresholding. After about $170 \mathrm{~ms}$ false tags deteriorate the analysis.

limited. We used this knowledge by passing circular frequency regions around the initial position of the eight peaks (figure 2.2). The radius of the region was 10 frequency pixels, with a smoothed edge by using Hanning windowing (Press et al., 1988). 


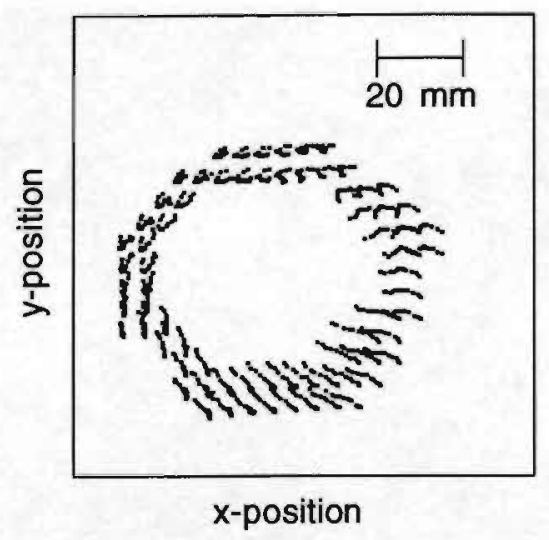

Figure 2.4 The positions of all tags in all frames of a scan covering isovolumic contraction and the early phase of ejection (20 to $210 \mathrm{~ms}$ after the R-wave in the ECG) of a healthy volunteer. Tag tracks were formed. Interruptions in the tracks were estimated assuming coherence of motion in the set of tags. Tracks with more than three estimated tag positions were excluded.

The filtered images (figure 2.3, left) were thresholded (figure 2.3, right) at a level of one third of the maximum gray value. Only tags with a size in the range of 5 to 50 pixels were considered for further analysis. Tag positions were calculated as the center of gravity of the pixels in a tag. Corresponding tags in consecutive images were identified by the Iterative Lower Rank approximation procedure developed by Muijtjens et al (Muijtjens et al., 1993). In this procedure coordinates of missing tags in the tracks are estimated assuming coherence of motion in the set of tags. Tracks with interruptions of more than three frames (images) were excluded from further analysis. Figure 2.4 shows tag tracks of a representative scan. For some scans tracks had to be excluded from the analysis because they did not belong to the left ventricular wall. We selected the tracks manually by indicating the inner and outer boundaries in a reference frame by two concentric circles. Note that the tags were detected and tag tracks were formed before this region of interest in the reference frame was indicated. So, when a tag was located within the region of interest in the reference frame, all tags in other frames belonging to the same track were known to be located within the left ventricular wall. 


\subsubsection{Kinematic analysis}

The tag positions were fitted to a modification of an earlier presented kinematic model of $2 \mathrm{D}$ cross-sectional deformation, based on known patterns of left ventricular motion (Arts et al., 1992). Basic modes of motion of the cross-section of the left ventricle are rigid body displacement and rotation, and cavity area ejection (concentric contraction). The latter is related to shortening of the inner circumference of the left ventricular wall. Myocardial infarction was assumed to induce lateral motion directed outward from the infarcted region (Bovendeerd et al., 1996). Added to the overall circumferential shortening, this means that circumferential shortening in the infarcted region is reduced. This effect was described by a sinusoidal deviation of the rotation angle from the average rotation along the circumference. Then, circumferential shortening is least at the maximum of the sine. The reference frame was chosen near mid-ejection. Motion parameters for all frames were estimated with respect to the geometry at this reference by means of optimization. Note that the two partially overlapping scans were processed independently.

The tag positions $\mathbf{x}_{r e f, i}$ in the reference configuration were described in polar coordinates $\left(r_{\text {ref }, i}, \phi_{\text {ref }, i}\right)$ around the center of the left ventricular cross-section $\mathbf{M}_{\text {ref }}$ :

$$
\mathbf{x}_{r e f, i}=\mathbf{M}_{r f f}+r_{r e f, i}\left[\begin{array}{c}
\cos \left(\phi_{r e f, i}\right) \\
\sin \left(\phi_{r e f, i}\right)
\end{array}\right]
$$

Then, tag positions $\mathbf{x}_{r e f,}$ in the reference configuration were displaced to $\hat{\mathbf{x}}_{t, d}$ depending on the motion parameter values $\Delta \mathbf{M}$, (rigid body displacement), $\Phi$, (rotation), $K_{t}$ (area ejection), $A S_{x, t}$ and $A S_{y, t}$ (x and y-component of asymmetry parameter, respectively):

$$
\hat{\mathbf{x}}_{t, j}=\mathbf{M}_{r e j}+\Delta \mathbf{M}_{t}+\sqrt{r_{r e f, ~}{ }^{2}+K_{t} / \pi}\left[\begin{array}{c}
\cos \left(\phi_{r e f, i}+\Phi_{t}+A S_{x, t} \sin \left(\phi_{r e f, j}\right)-A S_{y, t} \cos \left(\phi_{r e f, j}\right)\right) \\
\sin \left(\phi_{r e f, i}+\Phi_{t}+A S_{x, t} \sin \left(\phi_{r e f, i}\right)-A S_{y, d} \cos \left(\phi_{r e f, i}\right)\right)
\end{array}\right] .
$$

The asymmetry parameters $A S_{x, t}$ and $A S_{y, r}$ reflect regional differences in circumferential rotation. The parameter $A S_{x, t}$ represents stretching at the positive $x$-axis, while the parameter $A S_{y, 1}$ represents stretching at the positive $y$-axis. The combined effects of $A S_{x, s}$ and $A S_{y, s}$ result in stretching of the wall at angular position 
$\vartheta=\arctan 2\left(A S_{x, i}, A S_{y, s}\right)$ with strain amplitude $\sqrt{A S_{x, i}{ }^{2}+A S_{y, t}{ }^{2}}$, and contracting on the opposite side of the wall. Calculated tag positions $\hat{\mathbf{x}}_{r j}$ were compared with the measured tag positions $\mathbf{x}_{t, i}$ at time $t$. The sum of the squares of the distances between measured and calculated tag positions was minimized by estimation of the motion parameters for all moments in time and the positions of all considered tags in the reference configuration.

Computational effort was reduced by estimation of the motion parameters in each frame separately. An objective function $E_{1}$, representing the mean residual error between measured and calculated tag positions, was defined as:

$$
E_{t}=\frac{1}{N-1} \sqrt{\sum_{i=1}^{N}\left|\hat{\mathbf{x}}_{t, j}-\mathbf{x}_{t, j}\right|^{2}},
$$

with $\mathrm{N}$ the number of tags within the left ventricular cross-section. The objective function was minimized by variation of the time dependent motion parameters, being displacement $\Delta \mathbf{M}_{1}$, rotation $\Phi_{1}$, area ejection $K_{t}$, and the asymmetry parameters $A S_{x, t}$ and $A S_{y, t}$. The center $\mathbf{M}_{r e f}$ was defined as the center of the concentric circles serving as left ventricular boundaries at the reference time.

The positions of the tags in the reference configuration were re-estimated in a second stage. Each measured tag position was projected back on the reference frame applying the estimated deformation parameters in reverse order. Thus, in the reference frame for each tag a number of estimated positions was obtained, equal to the number of frames. The median $x$ and $y$-values per tag were used as the new reference position of that tag. Finally, the time-dependent motion parameters were reestimated using the new reference configuration. Optimization was performed with Powell's method (Press et al., 1988), which is the prototype of direction-set methods for minimization of an objective function.

The influence of inaccuracies in tag positions on the estimated motion parameters was assessed by adding noise to the positions of tags in a stochastic analysis. Tag positions in a reference frame were displaced according to parameter values in the physiologic range. Gaussian noise with a standard deviation of $0.3 \mathrm{~mm}$ was added to the positions in both frames, and subsequently the motion parameters were estimated 
and compared with the known values. This was repeated 1000 times. Standard deviations of the estimated parameter values were calculated.

From our models we found that during systole practically all kinematic parameters vary proportionally. A different pattern of contraction is reflected in a difference in the ratio of the changes in kinematic parameters. Therefore we normalized the asymmetry parameters to the ejected cavity area using linear regression:

$$
\begin{aligned}
& A S_{x, s}=\overline{A S}_{x} \frac{K_{f}}{A_{w}}+\text { constunt }_{x} \\
& A S_{y, s}=\overline{A S}_{y} \frac{K_{t}}{A_{n}}+\text { constant }_{y},
\end{aligned}
$$

with $\overline{A S}_{x}$ and $\overline{A S}_{y}$ the normalized (time-independent) parameters, and $A_{w}$ the wall area between inner and outer circles at the reference time. So, $\overline{A S}_{x}$ and $\overline{A S}_{y}$ are the slopes of the graphs of the asymmetry values against cavity area ejection normalized to wall area. Only the asymmetry parameter values during the ejection phase were used to determine the slopes. The normalized asymmetry vector $\left(\overline{A S}_{x}, \overline{A S}_{y}\right)$ points in the direction from where the outward circumferential motion originates, i.e. the direction where circumferential strain is least during ejection, and its length reflects the degree of asymmetry in the motion pattern.

\subsection{RESULTS}

The time required for analysis (determination of left ventricular contours at reference time, tag detection and tracking, parameter estimation) of the two sequential scans was about 10 minutes on a SUN Sparc IPX, manual actions being limited to a localization of the left ventricle in the reference image.

\subsubsection{Image analysis}

The accuracy of the calculated centers of gravity of the tags, as deduced from tag size, decreased from about $0.1 \mathrm{~mm}$ in the first images to about $0.3 \mathrm{~mm}$ in the last images of a scan. Tag tracks with a length of about $200 \mathrm{~ms}$, or 10-12 frames, were formed using the Iterative Lower Rank approximation procedure. About 5-10\% of the 

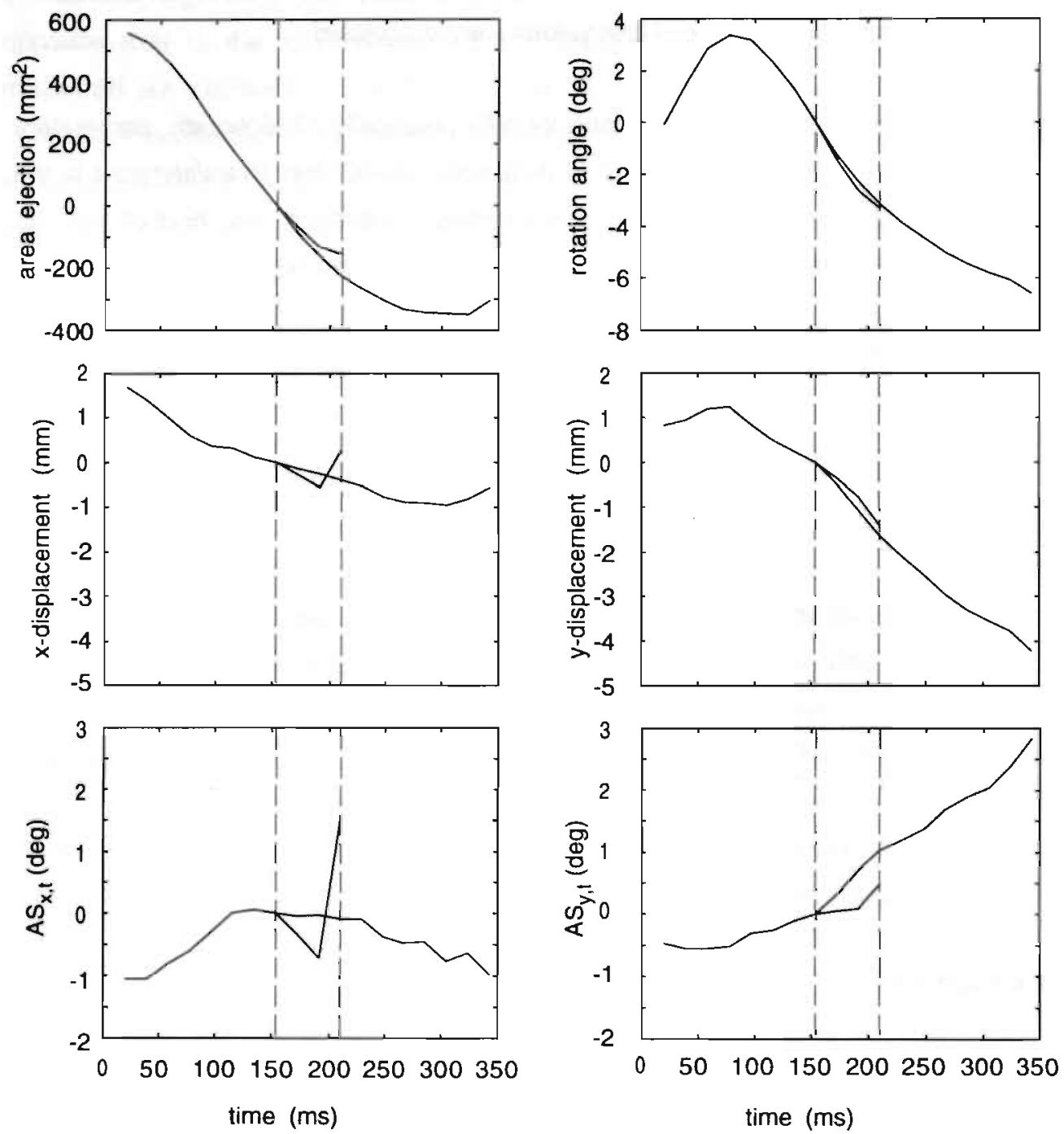

Figure 2.5 Estimated parameter values of a healthy volunteer as a function of time. Time 0 refers to the R-wave in the ECG. Asymmetry parameters are indicated by $A S_{x, t}$ and $A S_{y, r}$. Parameter values for two sequential scans are connected so that the entire ejection phase is covered. The period of overlap is indicated by the vertical dashed lines. The first dashed line indicates the start of the second scan.

data on tag positions were filled in by this procedure. Most of the data additions occurred in the last two images. 
Matched bandpass filtering enhanced the correct detection of the tags. More tags were correctly detected and less false detections occurred. The beneficial effect of filtering was more pronounced for the later, more faded images. Filtering extended the time that tags could be tracked by about 2 frames $(40 \mathrm{~ms})$.

\subsubsection{Kinematic analysis}

The estimated parameter values for two representative sequential scans of a volunteer are shown as a function of time in figure 2.5. From the time course of the parameter for area ejection $(K)$ in this figure we concluded that these scans covered the entire ejection phase. This was the case for most of the scans. Note that generally the isovolumic contraction phase lasts until about $60 \mathrm{~ms}$ after the R-wave in the ECG. During this phase the cavity area of the short-axis cross-section apparentiy diminishes due to shape change of the left ventricle while cavity volume remains approximately constant. Rotation is counterclockwise (upward slope) during isovolumic contraction and early ejection and reverses to clockwise (downward slope) during mid-ejection, as viewed from apex to base. This was found for all volunteers. In the following, $p$-values refer to the significance of differences in values between volunteers and patients. Maximum rigid body displacement of the left ventricular cross-section during the ejection phase was $3.8 \pm 1.4 \mathrm{~mm}$ (mean $\pm \mathrm{sd}$ ) for the 9 volunteers and $3.0 \pm 0.9 \mathrm{~mm}$ for the patients $(p=0.11)$. The ranges of rotation and area ejection averaged $6.4 \pm 1.5$ degrees and $945 \pm 71 \mathrm{~mm}^{2}$, respectively, for the volunteers, and $3.0 \pm 0.8$ degrees $(p<0.001)$ and $700 \pm 176 \mathrm{~mm}^{2}(p=0.02)$, respectively, for the patients. The maximum value of the amplitude of the asymmetry parameters $\left(\sqrt{A S_{x, t}{ }^{2}+A S_{y . t}{ }^{2}}\right)$ was $2.25 \pm 0.79$ degrees for the volunteers and $2.93 \pm 1.26$ degrees for the patients $(p=0.16)$.

Figure 2.6 shows the asymmetry parameters as a function of ejected cavity area over wall area for a volunteer and a patient. Linear regression was applied to these curves. Figure 2.7 shows for all volunteers and all patients the normalized asymmetry vector. The length of this vector averaged $5.0 \pm 1.9$ degrees for the volunteers and $11.1 \pm 4.3$ degrees for the patients $(p=0.02)$. 

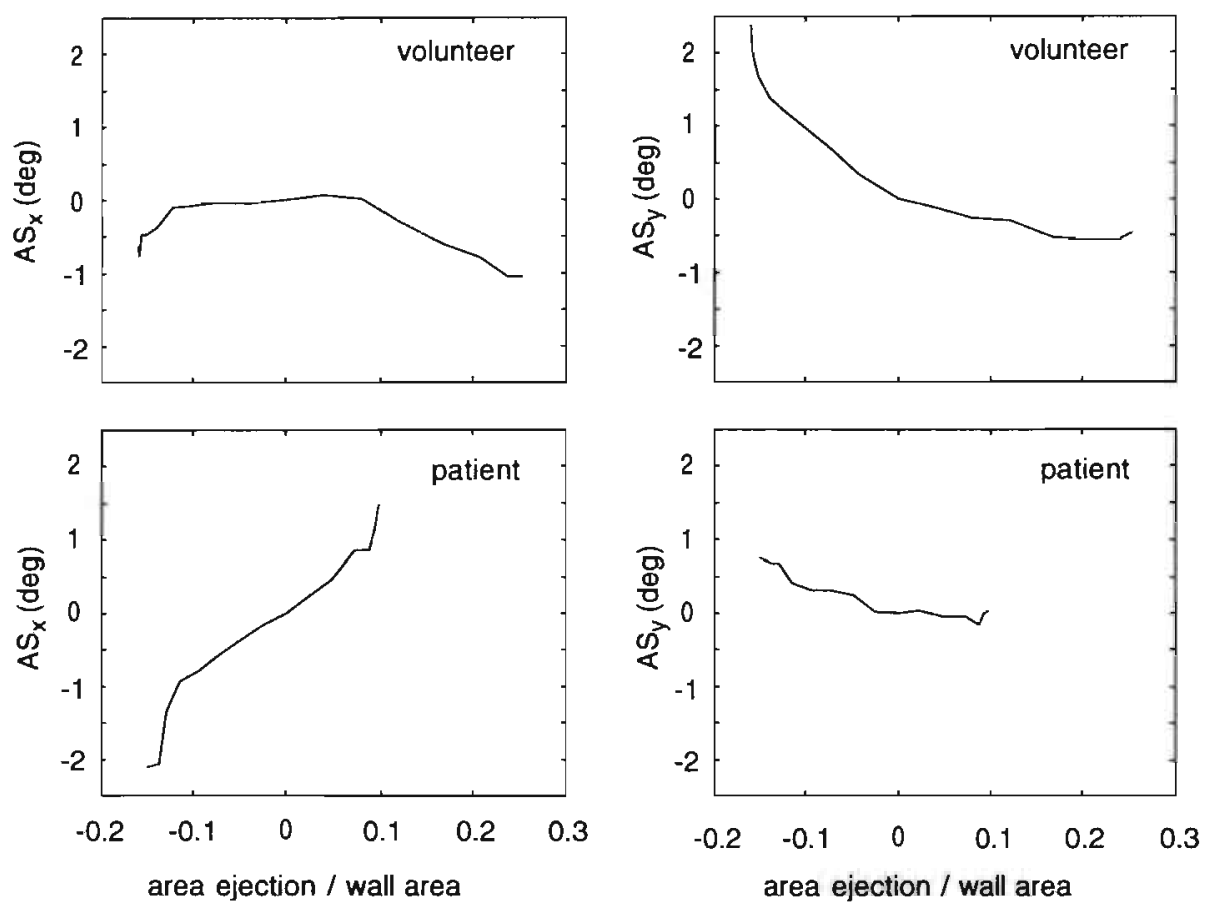

Figure 2.6 Asymmetry parameters ( $x$-and $y$-component) as a function of ejected cavity area over wall area. Top: volunteer, bottom: patient. Linear regression was applied to these curves and the slopes give the components of the normalized asymmetry vector $\left(\overline{A S}_{x}, \overline{A S}_{y}\right)$.

With the help of the stochastic simulation, using a standard deviation of the Gaussian noise applied to the tag positions of $0.3 \mathrm{~mm}$, standard deviations of the estimated parameter values were found to be $0.05 \mathrm{~mm}$ for displacement, 0.08 degrees for rotation, $6.2 \mathrm{~mm}^{2}$ for area ejection, and 0.16 degrees for the asymmetry parameters. Estimated motion parameter values for the two scans during the overlapping period generally differed. Standard errors between the parameter estimates of the last overlapping frame of the scans were $0.26 \mathrm{~mm}$ for displacements, 0.69 degrees for rotation angles, $35.9 \mathrm{~mm}^{2}$ for area ejection, and 0.60 degrees for asymmetry parameters. There was no significant bias between the two estimates. Parameter values determined using the first images of the second scan were, due to better image quality, more reliable than the parameter values obtained using the last images of the first scan. 


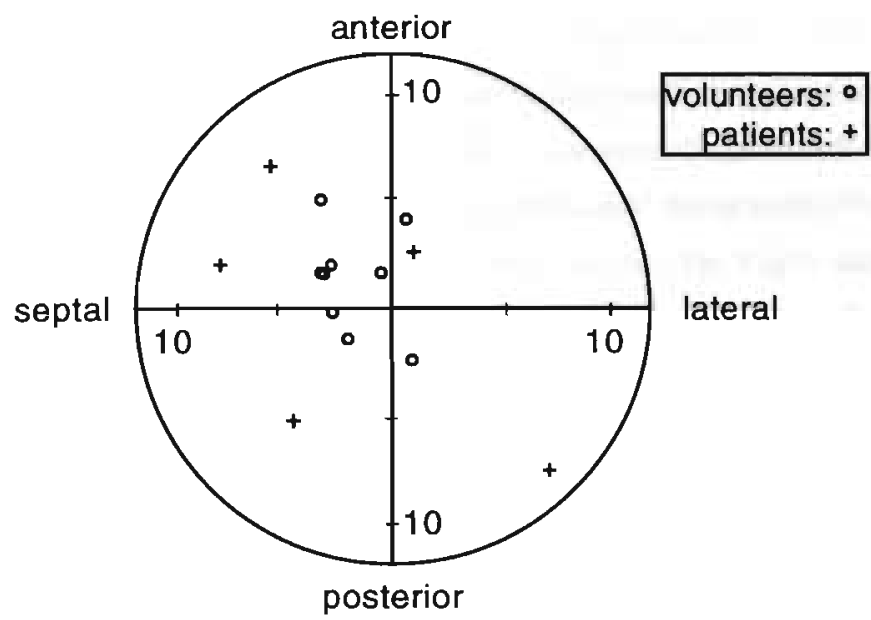

Figure 2.7 Normalized asymmetry vector $\left(\overline{A S}_{x}, \overline{A S}_{v}\right)$ in degrees, for all volunteers and all patients. The vector points to the region with minimum circumferential shortening and its length represents the degree of asymmetry. It can clearly be seen that the length of the vector is greater for the patients than for the volunteers. The patients were infarcted mainly in the anteroseptal region of the left ventricle, located in the upper-left quadrant of the figure.

\subsection{DISCUSSION}

A method is presented to assess noninvasively motion information of a cross-section of the left ventricle from MR-tagged images. For acquisition of local motion information of the myocardial wall the images were bandpass filtered and subsequently thresholded. So a high degree of automation of image analysis could be obtained, which is necessary for possible future routine clinical applications. A kinematic model of left ventricular deformation was used to investigate the pattern of motion. In addition to parameters describing the most basic normal modes of motion, parameters describing the specific influence of regional myocardial dysfunction were incorporated into the kinematic model. Significant differences $(p=0.02)$ in normalized asymmetry vectors were found between volunteers and patients. For an accurate description of the influences of pathologies on wall deformation an extension of the method to $3 \mathrm{D}$ is necessary, requiring image acquisition from multiple short-axis and long-axis cross-sections. 


\subsubsection{Analysis and results}

Filtering of the images before thresholding appears to be useful. Tags fade away while noise in the images increases. Inside the cavity of the left ventricle the tags disappear shortly after excitation of the grid due to the flowing of blood. This results in a relatively large bright area in the ventricular cavity. The developed filtering method reduces the effect of fading of the tags and removes the unwanted low spatial frequencies. Spatial frequencies were passed by the filter that account for strains of up to $30 \%$ and rotations of up to 20 degrees, well within limits of measured local deformations in the left ventricle. However, it may be necessary to adjust the filter when investigating cardiac diseases with more severe alterations of the myocardial deformation pattern. After filtering the images were much more suitable for thresholding. In processing the images the use of a fixed threshold level was found to be satisfactory.

The asymmetry parameters describe the location and amplitude of a less contracting region in its simplest form. From model studies (Bovendeerd et al., 1996) as well as $2 \mathrm{D}$-echocardiographic registrations it is known that the short-axis cross-section remains circular, even if part of the circumference is not contracting. A lesser contracting region is mainly recognized by lateral displacements in the wall. In the study by Bovendeerd et al (Bovendeerd et al., 1996) lateral extension propagated further into the healthy region than wall thinning. Thus by measuring lateral wall extension the chance to miss aberrations in mechanical performance diminishes.

Differences were found between the estimated motion parameters of the two scans during the time overlap. The ratios of the standard errors between the parameter estimates of the last overlapping frames to the standard deviations of the parameters as found by the stochastic analysis were determined. These ratios were comparable in magnitude for all six parameters, indicating that the standard errors between the estimates of the two scans are likely to be related to inaccuracies in the measurement of tag positions. Furthermore, subject motion between the two scans can cause differences in parameter estimates.

There are no significant differences in asymmetry parameters $A S_{x, s}$ and $A S_{y, t}$ of volunteers and patients. However, our model studies showed that a different pattern 
of deformation is reflected most evidently in a difference in the ratio of the changes in kinematic parameters. The length of the asymmetry vector, which is normalized to the ratio of ejected cavity area to wall area, did show significant differences between volunteers and patients $(p=0.02)$. So, relative to the overall contraction of the heart asymmetrical circumferential shortening was increased in the patients. Furthermore, it should be noted that besides the length of the normalized asymmetry vector, its direction is also important: theoretically it should point in the direction of the infarction. The patients were known to be infarcted in the anteroseptal region of the left ventricle. Because only one left ventricular cross-section was imaged, 3D motion information is limited. As a result the influence of the infarcted region can modify the direction of the vector. The normalized asymmetry vector of one patient was in the same range as those of the volunteers. This may be due to the circumstance that the imaged crosssection did not come near the infarcted region. For a more accurate description of the influence of myocardial infarction, the method needs to be extended to 3 dimensions, i.e., more cross-sections need to be imaged.

\subsubsection{Comparison with previous studies}

We have used thresholding to identify corresponding tags in consecutive images. Our matched filtering technique facilitates thresholding significantly. In other studies (Clark et al., 1991; Maier et al., 1992; Guttman et al., 1994; Kumar and Goldgof, 1994; Young et al., 1994; Kraitchman et al., 1995) no threshold was used but the crossingpoints of the dark grid lines in the images were determined. In this approach full automation appears to be more complicated, often requiring manual adjustments.

Kraitchman et al (Kraitchman et al., 1995) used a semi-automatic method for tracking of myocardial motion in MR-tagged images using active contour modeling or "snakes". Analysis of 25 short-axis images took about 25 minutes of user interface time on an SGI Indigo workstation, which amounts to approximately 1 minute per image. Our method detected and tracked tags with an average time of less than 10 seconds per image on a SUN Sparc IPX. Although Kraitchman et al extracted more information about local deformation, our method has the advantage that considerably less user-interaction is needed. 
Young et al (Young et al., 1994) and O'Dell et al (O'Dell et al., 1995) also used models to fit tag location data. They described the motion field by an analytic series expansion, with which they obtained a 3D tracking error of less than $0.1 \mathrm{~mm}$. We focused our analysis on asymmetric contraction of the left ventricle due to myocardial infarction. Myocardial infarction influences the deformation of the left ventricle over a large region extending beyond the boundaries of the infarcted region (Kramer et al., 1996; Marcus et al., 1997). So, a global kinematic model describing the deformation of the left ventricle may be of help to diagnose regional contractile disorders.

When using more advanced tagging techniques, in which tagging contrast is enhanced (Fischer et al., 1993), using breath-hold MRI (McVeigh and Atalar, 1992), or using echo-planar imaging (Tang et al., 1995), the time span that tags can be followed accurately can be extended. An elaborate optimization of the tagging scheme, however, was not at our disposal because of the limited accessibility of our MR Imaging system, and was not the focus of our study. Furthermore, we showed that with the help of a kinematic model image series partially overlapping in time can be connected. Thus, in studying cardiac motion a limitation in tag observation time can be overcome.

We fitted the tag tracks to a kinematic model in order to estimate displacement, rotation, radial motion, and asymmetry parameters. The center of the cross-section was determined visually at one single reference time, by indicating a pair of concentric circles representing left ventricular contours. The motion of the center was determined objectively on the basis of tag motion. Thus manual (Young et al., 1994) assessment of the center at all times or the use of a fixed center (Maier et al., 1992; Qi et al., 1993) was avoided. Furthermore, it should be noted that with tag detection methods using snakes accurate delineation of left ventricular boundaries at all times is necessary to avoid errors in the fitted displacement field. Our method only requires indication of those tags which belong to the left ventricular cross-section. Therefore, an approximate delineation of contours suffices. This needs to be done only at one reference time since tag tracks are formed independently.

We acquired short-axis images at only one long-axis location. Therefore the nature of our analysis is two-dimensional. Through-plane motion cannot be accounted for in this set-up. Rogers et al (Rogers et al., 1991) quantified left ventricular systolic long- 
axis shortening. They reported a total change in position along the long axis between end-diastole and end-systole of $12.8 \pm 3.8 \mathrm{~mm}$ at the base, $6.9 \pm 2.6 \mathrm{~mm}$ at the mid left ventricular level and $1.6 \pm 2.2 \mathrm{~mm}$ at the apex. We acquired images at about the midventricular level. The through-plane motion is of the same order as the slice thickness. This limits the accuracy with which tags can be followed in two dimensions. Three-dimensional imaging (Young and Axel, 1992; Azhari et al., 1993) would avoid this source of inaccuracy.

At present, a shortcoming of our method is the low spatial resolution with which influences of pathologies on the deformation pattern can be described. This problem can be overcome by an extension of the number of cross-sections. Images from short-axis and long-axis cross-sections will have to be combined to determine 3D wall deformation. Present methods to determine 3D wall deformation have the disadvantage that a large amount of user-interaction is needed to analyze the images. Our method has the potential to determine similar 3D deformation information with a high degree of automation, making it more suitable for routine clinical applications.

\subsubsection{Conclusions}

MR-tagging is a noninvasive method by which a grid of tags can be attached to the myocardial tissue. The grid deforms with the tissue and its motion can be followed in time. Spatial bandpass filtering and subsequent thresholding of the images facilitated automatic detection of the tags. A kinematic model of cardiac motion is a useful tool for recognizing patterns in cardiac deformation. The kinematic model also enables analysis of cardiac motion over longer periods of time by connecting separate shorter periods of acquired motion information. In analyzing the motion of a cardiac crosssection in volunteers and patients with infarction, significant differences were found in motion asymmetry. We expect that by analyzing images of more cross-sections simultaneously, more details in motion can be recognized efficiently. 


\section{REFERENCES}

Arts, T., Hunter, W. C., Douglas, A., Muytjens, A. M. M., and Reneman, R. S. (1992) Description of the Deformation of the Left Ventricle by a Kinematic Model. $J$ Biomechanics 25, 1119-1127.

Axel, L., Bloomgarden, D. C., Chang, C.-N., Fayad, Z. A., Kraitchman, D. L., and Young, A. A. (1995) An integrated program for 2-D and 3-D analysis of heart wall motion from Magnetic Resonance Imaging. In Computers in Cardiology. Vienna: IEEE.

Axel, L. and Dougherty, L. (1989) MR Imaging of Motion with Spatial Modulation of the Magnetization. Radiology 171, 841-845.

Axel, L., Gonçalves, R. C., and Bloomgarden, D. (1992) Regional Heart Wall Motion: Two-dimensional Analysis and Functional Imaging with MR Imaging. Radiology 183, 745-750.

Azhan, H., Weiss, J. L. Rogers, W. J., Siu, C. O., Zemoun, E. A., and Shapiro, E. P. (1993) Noninvasive Quantification of Principal Strains in Nomal Canine Hearts using Tagged MRI images in 3-D. Am J Physiol 264, H205-H216.

Bovendeerd, P. H. M., Arts, T., Delhaas, T.x Huyghe, J. M., Van Campen, D. H., and Reneman, R. S. (1996) Regional wall mechanics in the ischemic left ventricle: numerical modeling and dog experiments. Am J Physiol 270, H398-H410.

Clark, N. R., Reichek, N., Bergey, P., Hoffman, E. A., Brownson, D., Palmon, L., and Axel, L. (1991) Circumferential Myocardial Shortening in the Normal Human Left Ventricle - Assessment by Magnetic Resonance Imaging Using Spatial Modulation of Magnetization. Circulation $84,67-74$.
Fischer, S. E., McKinnon, G. C., Maier, S. E., and Boesiger, P. (1993) Improved Myocardial Tagging Contrast. Magn Reson Med 30, 191-200.

Guttman, M. A., Prince, J. L., and McVeigh, E. R. (1994) Tag and contour detection in tagged MR images of the left ventricle. IEEE T Med Imaging 13, 74-88.

Kraitchman, D. L., Young, A. A., Chang, C.-N., and Axel, L. (1995) Semi-automatic tracking of myocardial motion in MR-tagged images. IEEE T Med Imaging 14, 422-433.

Kramer, C. M., Rogers, W. J., Theobald, T. M., Power, T. P., Petruolo, S., and Reichek, N. (1996) Remote noninfarcted region dysfunction soon after first anterior myocardial infarction. A Magnetic Resonance Tagging study. Circulation 94, 660-666.

Kumar, S. and Goldgof, D. (1994) Automatic tracking of SPAMM grid and the estimation of deformation parameters from cardiac MR images. IEEE T Med Imaging 13, 122132.

Lessick, J., Sideman, S., Azhañ, H., Shapiro, E., Weiss, J. L., and Beyar, R. (1993) Evaluation of regional load in accute ischemia by three-dimensional curvatures analysis of the left ventricle. Ann Biomed Eng 21, 147-161.

Lima, J. A. C., Ferrani, V. A, Reichek, N., Kramer, C. M., Palmon, L., Llaneras, M. F. Tallant, B., Young, A. A., and Axel, L. (1995) Segmental motion and deformation of transmurally infarcted myocardium in acute postinfarct period. Am J Physiol 268, $\mathrm{H} 1304-\mathrm{H} 1312$

Maier, S. E., Fischer, S. E., McKinnon, G. C., Hess, O. M., Krayenbuehl, H. P., and 
Boesiger, P. (1992) Evaluation of Left Ventricular Segmental Wall Motion in Hypertrophic Cardiomyopathy with Myocardial Tagging. Circulation 86, 19191928.

Marcus, J. T., Gotte, J. W., Van Rossum, A. C., Kuijer, J. P. A., Heethaar, R. M., Axel, L., and Visser, C. A. (1997) Myocardial function in infarcted and remote regions early after infarction in man: Assessment by Magnetic Resonance tagging and strain analysis. MRM 38, 803-810.

McVeigh, E. R. (1996) MRI of myocardial function: motion tracking techniques. Magn Reson Imaging 14, 137-150.

McVeigh, E. R. and Atalar, E. (1992) Cardiac Tagging with Breath-Hold Cine MRI. MRM 28, 318-327.

Muijtiens, A. M. M., Roos, J. M. A., Arts, T., Hasman, A., and Reneman, R. S. (1993) Extrapolation of Incomplete Marker Tracks by Lower Rank Approximation. Int $J$ Biomed Comput 33, 219-239.

O'Dell, W. G., Moore, C. C., Hunter, W. C., Zerhouni, E. A., and McVeigh, E. R. (1995) Three-dimensional myocardial deformations: calculation with displacement field fitting to tagged MR images, Radiology $195,829-835$.

Press, W. H., Flannery, B. P., Teukolsky, S. A., and Vetterling, W. T. (1988) Numerical recipes - The art of scientific computing. Cambridge University Press, Cambridge.

Prinzen, F. W., Arts, T., Hoeks. A. P. G., and Reneman, R. S. (1989) Discrepancies between Myocardial Blood Flow and Fiber Shortening in the Ischemic Border Zone as assessed with Video Mapping of Epicardial
Deformation. European $J$ of Physiology 415, 220-229.

Qi, P., Thomsen, C., Stáhlberg, F., and Henriksen, O. (1993) Normal Left Ventricular Wall Motion Measured with Two-dimensional Myocardial Tagging. Acta Radiol 34, 450-456.

Rogers, W. J., Shapiro, E. P., Weiss, J. L., Buchalter, M. B., Rademakers, F. E., Weisfeldt, M. L., and Zerhouni, E. A. (1991) Quantification of and Correction for Left Ventricular Long-Axis Shortening by Magnetic Resonance Tissue Tagging and Slice Isolation. Circulation 84, 721-731.

Tang, C., McVeigh, E. R., and Zerhouni, E. A. (1995) Multi-shot EPI for improvement of myocardial tagging contrast: comparison with segmented SPGR. MRM 33, 443-447.

Young, A. A. and Axel, L. (1992) Threedimensional motion and Deformation of the Heart Wall: Estimation with Spatial Modulation of Magnetization - A Modelbased Approach. Radiology 185, 241-247.

Young, A. A., Imai, H., Chang, C. N., and Axel, L. (1994) Two-dimensional Left Ventricular Deformation during Systole using Magnetic Resonance Imaging with Spatial Modulation of Magnetization. Circulation 89, 740-752.

Young, A. A., Kramer, C. M., Ferrari, V. A., Axel, L., and Reichek, N. (1994) Threedimensional left ventricular deformation in hypertrophic cardiomyopathy. Circulation $90,854-867$.

Zertrouni, E. A., Parish, D. M., Rogers, W. J., Yang, A., and Shapiro, E. A. (1988) Human Heart: Tagging with MR Imaging - A Method for Noninvasive Assessment of Myocardial Motion. Radiology 169, 59-63. 



\title{
Chapter 3
}

Relation between torsion and cross-sectional area change in the human left ventricle

\author{
F.W.L. Aelen ${ }^{1}$, T. Arts ${ }^{1}$, D.G.M. Sanders ${ }^{4}$, G.R.P. Thelissen ${ }^{4}$, A.M.M. Muijtjens ${ }^{3}$, \\ F.W. Prinzen ${ }^{2}$, R. S. Reneman ${ }^{2}$
}

Departments of Biophysics ${ }^{1}$, Physiology ${ }^{2}$, and Medical Informatics ${ }^{3}$ Cardiovascular Research Institute Maastricht, Maastricht University, PO Box 616, 6200 MD Maastricht, The Netherlands,

Department of Diagnostic Radiology ${ }^{4}$, University Hospital Maastricht, PO Box 5800 , 6202 AZ Maastricht, The Netherlands.

J Biomechanics (1997), Vol. 30, No. 3: 207-212 


\subsection{ABSTRACT}

During the ejection phase motion of the left ventricular (LV) wall is such that all myocardial fibers shorten to the same extent. In a mathematical model of LV mechanics it was found that this condition could be satisfied only if torsion around the long axis followed a unique function of the ratio of cavity volume to wall volume. When fiber shortening becomes non-uniform due to cardiac pathology, this pathology may be reflected in aberration of the torsional motion pattern. In the present study we investigated whether the predicted regular motion pattern could be found in 9 healthy volunteers, using Magnetic Resonance Tagging. In two parallel short-axis crosssections displacement, rotation, and area ejection were derived from the motion of tags, attached noninvasively to the myocardium. Information from both sections was combined to determine area ejection, quantified as the change in the logarithm of the ratio of cavity area to wall area, and torsion, represented by the shear angle on the epicardium. Linear regression was applied to torsion as a function of area ejection. The slope thus found $(-0.173 \pm 0.024 \mathrm{rad}$, mean $\pm \mathrm{sd})$ was similar to the slope as predicted by the model of LV mechanics $(-0.194 \pm 0.026 \mathrm{rad})$. In conclusion, the relation between area ejection and torsion could be assessed noninvasively in humans. In healthy volunteers, the relation was close to what was predicted by a mathematical model of LV mechanics, and also close to what was found earlier in experiments on animals. 


\subsection{INTRODUCTION}

During contraction of the left ventricle (LV) circumferential strain in the subendocardial layers is about twice as much as in the subepicardial layers (Clark et al., 1991). The arrangement of myocardial fibers, however, is such that during ejection the transmural distribution of strain along the fiber direction is practically uniform (Arts and Reneman, 1989). The equalization of strain is a direct consequence of torsion of the wall around the long axis, and works as follows. In the subendocardial and subepicardial layers the fibers follow helical pathways with mutually opposite pitch direction. During ejection torsion uncoils the subepicardial helices and coils the subendocardial ones. As a consequence, due to torsion subepicardial fibers experience extra shortening at the cost of lengthening of the subendocardial fibers. Thus, torsion is a means to redistribute strain over the wall. Without torsion the subendocardial fibers would shorten much more than the subepicardial ones. In a mathematical model of LV wall mechanics (Arts et al., 1984; Arts and Reneman, 1989) it is found that appropriate tuning of torsion to the ejected volume can cancel these transmural differences. In animal experiments the predicted relation between torsion and ejected volume is confirmed to exist (Arts et al., 1984).

Cardiac disorders such as myocardial ischemia or infarction, and hypertrophic cardiomyopathy have been shown to alter the pattern of myocardial deformation (Prinzen et al., 1989; Maier et al., 1992; Young et al., 1994; Lima et al., 1995). Such disorders cause non-uniformities in fiber shortening, which likely results in disruption of the fine tuning of torsion to changes in ejected volume. We propose to use our foreknowledge about regular cardiac motion to enhance sensitivity in detecting motion disorders. As a first step the normal pattern of contraction has to be assessed with tools available to the clinic.

Developments in Magnetic Resonance Imaging (MRI) have provided powerful tools to quantify LV deformation (Zerhouni et al., 1988; Buchalter et al., 1990; Maier et al., 1992; Young et al., 1994). With the Magnetic Resonance Tagging technique a grid of tags is attached noninvasively to the heart by means of Spatial Modulation of the Magnetization (SPAMM). The grid deforms with the tissue and can be visualized for a period of about $300 \mathrm{~ms}$ (figure 3.1). This period is generally long enough to cover the ejection phase. 


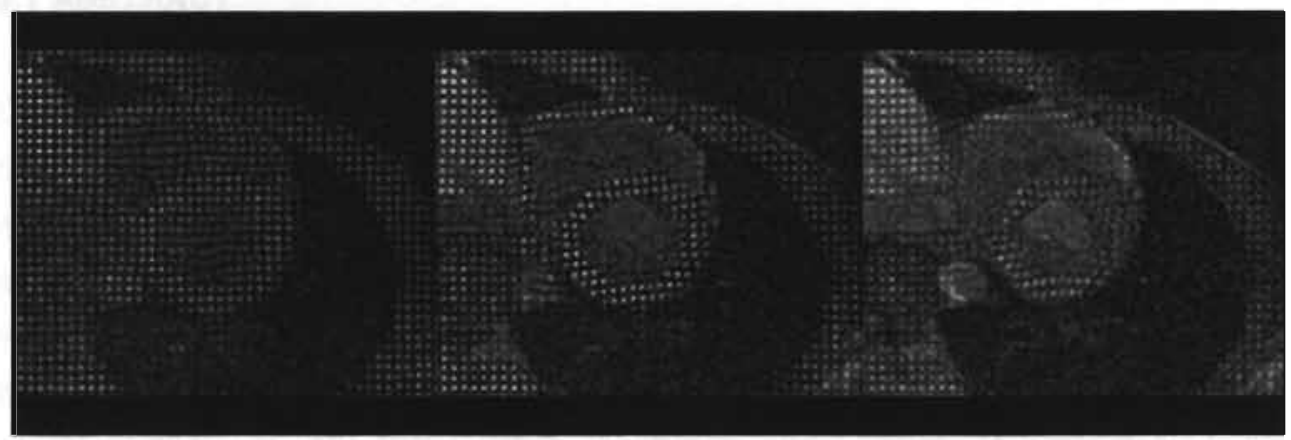

Figure 3.1 MR-tagged short-axis images of the contracting cardiac left ventricle. Small regions of altered magnetization are visible as tags. The images were acquired at 20,96, and $172 \mathrm{~ms}$ after the R-wave in the ECG. The motion of the tags corresponds to myocardial deformation. Gradually the tags fade away. In the left ventricular cavity they disappear shortly after excitation of the grid due to the ejection of blood.

The aim of this study was to determine the magnitude and range of the normal pattern of cardiac deformation, as expressed in terms of torsion and changes in crosssectional area of the LV. In healthy volunteers cardiac motion was measured with MR tagging. A kinematic model was used to extract the change in cavity area and torsion from the motion data. To judge our understanding of cardiac motion, the deformation as measured was compared with the deformation as predicted by a mathematical model of LV wall mechanics.

\subsection{MR MEASUREMENTS}

MR-tagged images were obtained in 9 healthy volunteers at two parallel short-axis cross-sections, located at about 2 and $4 \mathrm{~cm}$ below the base of the LV, using a 0.5 Tesla MR imaging system (Philips Gyroscan T5-II, Philips Medical Systems, Best, The Netherlands). The locations of these cross-sections were determined using a compound angulation method in which transverse and coronal-angulated images were acquired sequentially. Slice thickness was $8 \mathrm{~mm}$. From both cross-sections two sequential image series were acquired in order to cover the entire ejection phase. The applied inter-tag distance was $5 \mathrm{~mm}$ and the time interval between two consecutive 
images was about $20 \mathrm{~ms}$. In addition, non-tagged MR images were obtained at the same short-axis cross-sections.

Tags, defined as the bright spots in between the dark lines, were enhanced by matched spatial bandpass filtering of the separate images. In the 2-dimensional spatial frequency domain the information of the deformed grid of tags was restricted to regions around the lowest harmonics, i.e., eight regions arranged symmetrically around the origin. Only these regions were passed by the filter. The lowest spatial frequencies located around the origin, responsible for the large bright area in the LV cavity, were also filtered out. After filtering the images were thresholded. The position of a tag was calculated as the center of gravity of the pixels of the thresholded tag. Corresponding tags in consecutive images were identified by the Iterative Lower Rank Approximation procedure developed by Muijtjens et. al. (Muijtjens et al., 1993). By this procedure, coordinates of missing tags in the tracks were estimated assuming coherence of motion in the set of tags.

The tag tracks thus found (figure 3.2) were fitted to a kinematic model of LV crosssectional motion using a least squares method. In the kinematic model it was assumed that normal LV deformation is rotationally symmetric, wall volume is constant, and during contraction shortening of base to apex segment length is $65 \%$ of outer circumference shortening (Feigl and Fry, 1964). Then, the radial motion of all points in a cross-section of the wall can be described completely by the motion of the outer radius. At a conveniently chosen reference time the inner and outer radii of the LV were measured directly in the non-tagged images of both cross-sections. The reference time was chosen at about mid ejection, so that parameter values were estimated with respect to the tag positions in the middle of the trajectory of deformation.

For a mathematical description of the deformation we represent LV cross-sectional displacement by $\Delta \mathbf{M}_{t}$, rotation angle by $\Phi_{1}$, and the outer radius by $R_{o, t}$. A point in the LV wall with position vector $\mathbf{x}_{r e f}$ at the reference time (ref) moves to the position $\hat{\mathbf{x}}_{t}$ at time $t$ according to the kinematic model (Appendix A): 


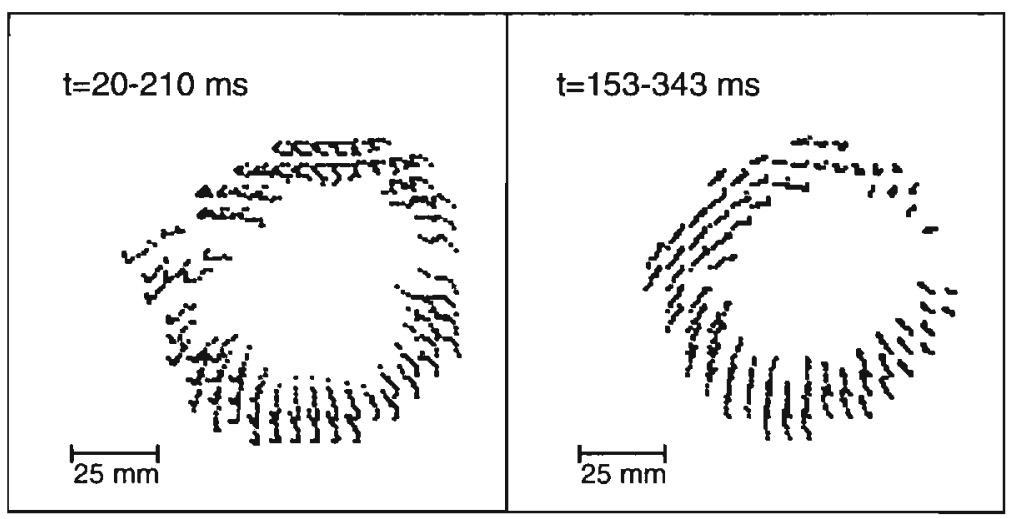

Figure 3.2 Tags in MR-tagged short-axis images of the ejecting human left ventricle were followed in time. The position of a tag is represented by its center of gravity in a thresholded image. Left panel: tag positions in a series of images covering the early phase of ejection (20 to $210 \mathrm{~ms}$ after the R-wave in the ECG). Three of the images in this series were shown in figure 3.1. Right panel: tag positions in a series of images covering the late phase of ejection (153 to $343 \mathrm{~ms}$ after the R-wave in the ECG). The tagging grid is rectangular in the first image of both series. Then the grid deforms along with the tissue. The general motion of the tags is inward, so time can be followed in the figures by following the tracks inward.

$$
\hat{\mathbf{x}}_{t}=\mathbf{M}_{r e f}+\Delta \mathbf{M}_{t}+\sqrt{R_{o, t}{ }^{2}-\left(R_{o, \text { ref }}{ }^{2}-r_{\text {ref }}{ }^{2}\right)\left(\frac{R_{o, r e f}}{R_{o, t}}\right)^{0.65}}\left[\begin{array}{c}
\cos \left(\phi_{r e f}+\Phi_{t}\right) \\
\sin \left(\phi_{r e f}+\Phi_{t}\right)
\end{array}\right] .
$$

The symbols $\left(r_{r e f}, \phi_{\text {ref }}\right)$ refer to the polar coordinates of $\mathbf{x}_{r e f}$ with respect to the center $\left(\mathbf{M}_{\text {ref }}\right)$ of the LV cross-section at the reference time. Thus calculated positions of all tags were compared with the measured positions $\left(\mathbf{x}_{t}\right)$ of the tags at time $t$ and a least squares fit was used to estimate the kinematic parameters $\mathbf{M}_{r e f}, \Delta \mathbf{M}_{t}, \Phi$, and $R_{o, x}$. Note that the two sequential image series were treated separately, each with its own reference tag positions. The reference time was the same for both image series.

After the kinematic parameters were estimated for both cross-sections, torsion and area ejection of the LV were quantified. Torsion was calculated as the axial gradient in rotation angle multiplied by the average of the outer radii in both sections: 
Torsion $_{t}=\frac{\Phi_{t}^{l}-\Phi_{t}^{u}}{Z} \cdot\left(R_{u, t}^{l}+R_{o, s}^{u}\right) / 2$

with $u$ and $l$ denoting the upper and lower LV cross-section, respectively, and $Z$ is the distance between the cross-sections. Physically, this measure of torsion may be interpreted as the shear angle on the epicardial surface between both cross-sections. LV area ejection was quantified as the natural logarithm of the ratio of cavity area $\left(A_{\varepsilon}\right)$ to wall area $\left(A_{w}\right)$ and was calculated using the average inner $\left(R_{i, t}\right)$ and outer $\left(R_{o . t}\right)$ radii in the two cross-sections:

$$
\ln \left[\frac{A_{c}}{A_{w}}\right]_{t}=\ln \left[\frac{\left(R_{t, t}^{l}+R_{i, s}^{u}\right)^{2}}{\left(R_{o, I}^{l}+R_{o, t}^{u}\right)^{2}-\left(R_{i, t}^{l}+R_{i, s}^{u}\right)^{2}}\right],
$$

The inner radius was determined as a function of the estimated outer radius by (Appendix A):

$$
R_{i, t}=\sqrt{R_{o, t}{ }^{2}-\left(R_{o, \text { ref }}{ }^{2}-R_{i, r e f}{ }^{2}\right)\left(\frac{R_{0, r e f}}{R_{o, t}}\right)^{0.65}} .
$$

Note that the inner and outer radii are measured once at the reference time. For the other moments in time these radii are calculated from the changes in position of the tags, assuming that the radii follow the motion of the grid of tags.

\subsection{MATHEMATICAL MODEL OF LEFT VENTRICULAR MECHANICS}

Previously, torsion and area ejection of the contracting LV were also calculated using a mathematical model of LV wall mechanics (Arts and Reneman, 1989). In this model the LV was represented by a thick-walled anisotropic cylinder, including the mitral valve - papillary muscle system. Fiber orientation in the wall was chosen such that the transmural distribution of fiber stress was as uniform as possible. The resulting transmural course of muscle fiber orientation was not significantly different from anatomical findings. The used relation between stress and strain in the myocardial tissue was physiological. Furthermore, the LV was loaded with a physiological 
hemodynamic impedance. LV geometry and hemodynamic variables were calculated throughout the cardiac cycle using equilibria of forces and torques.

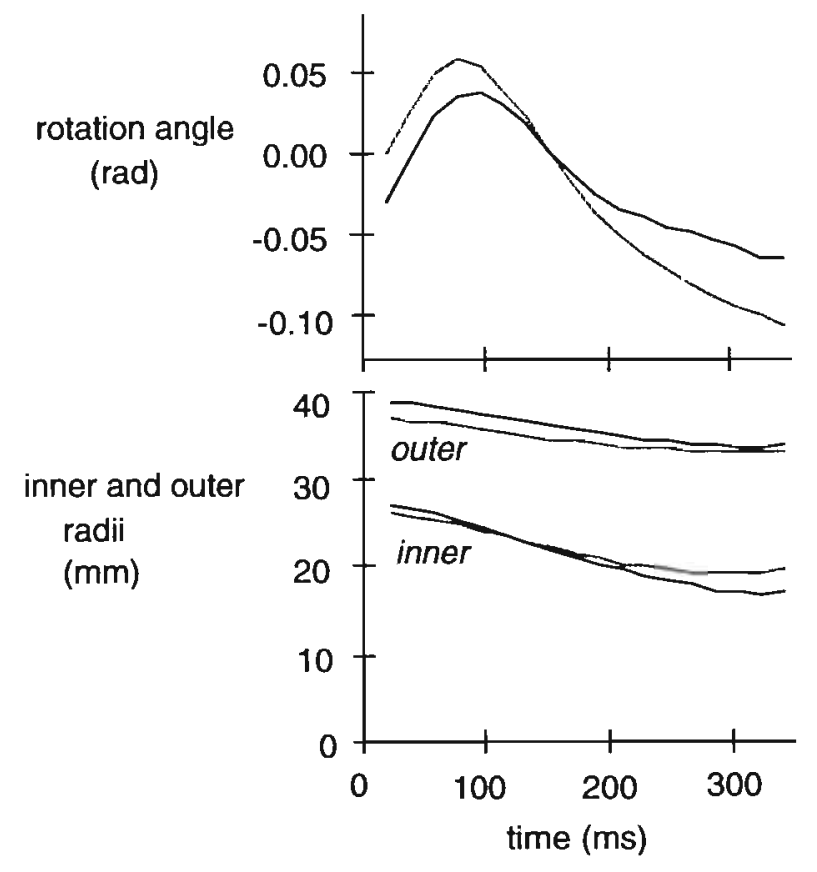

Figure 3.3 Rotation angles and inner and outer radii of two left ventricular short-axis crosssections as a function of time for a healthy volunteer. Time $O$ refers to the $R$-wave in the ECG. Dashed lines: upper cross-section. Solid lines: lower cross-section. Rotation angles are set to zero at the reference time at about mid ejection. Differences in rotation angles between the cross-sections are associated with torsion of the left ventricle.

\subsection{RESULTS}

MR-tagged images were obtained in 9 healthy volunteers at two parallel short-axis cross-sections located in the equatorial region of the LV. For each cross-section the displacement, rotation and area ejection parameters were estimated. Figure 3.3 shows examples of time courses of rotation angles and inner and outer radii. The ejection phase was assumed to be the period during which the inner radius decreased. Rotation angles from the beginning to the end of ejection were 


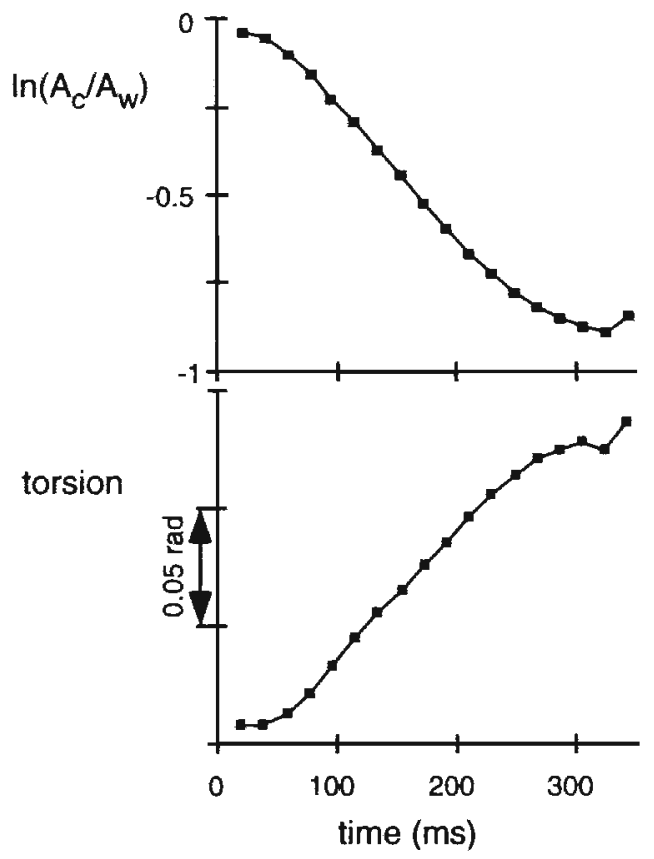

Figure 3.4 Time courses of area ejection (upper panel) and torsion (lower panel) of the left ventricle as measured by means of MRI. Time 0 refers to the R-wave in the ECG. Area ejection was expressed as the logarithm of the ratio of cavity area $\left(A_{f}\right)$ to wall area $\left(A_{w}\right)$. Torsion was represented by the shear angle on the epicardium. Only changes in the torsion angle are considered relevant.

$-0.056 \pm 0.049 \mathrm{rad}$ (mean $\pm \mathrm{sd}, \mathrm{n}=9$ ) for the upper cross-section, and $0.035 \pm 0.054 \mathrm{rad}$ in the opposite direction for the lower cross-section, as viewed from apex to base, counterclockwise positive. The outer radius decreased from $36.0 \pm 2.7 \mathrm{~mm}$ to $32.3 \pm 2.7$ $\mathrm{mm}$ for the upper cross-section, and from $35.4 \pm 3.0 \mathrm{~mm}$ to $31.4 \pm 2.5 \mathrm{~mm}$ for the lower cross-section. The inner radii decreased relatively more: from $25.2 \pm 1.5 \mathrm{~mm}$ to $18.1+2.2 \mathrm{~mm}$ for the upper cross-section, and from $23.9 \pm 2.1 \mathrm{~mm}$ to $15.5 \pm 2.0 \mathrm{~mm}$ for the lower one. Torsion and area ejection were calculated as a function of time (figure 3.4). Note the similarity in the time courses of torsion and the logarithm of normalized cavity area during the ejection phase, except for the sign. During ejection, torsion increased by an amount of $0.146 \pm 0.028 \mathrm{rad}$. The ratio of cavity area to wall area decreased from $0.92 \pm 0.16$ to $0.41 \pm 0.12$. 


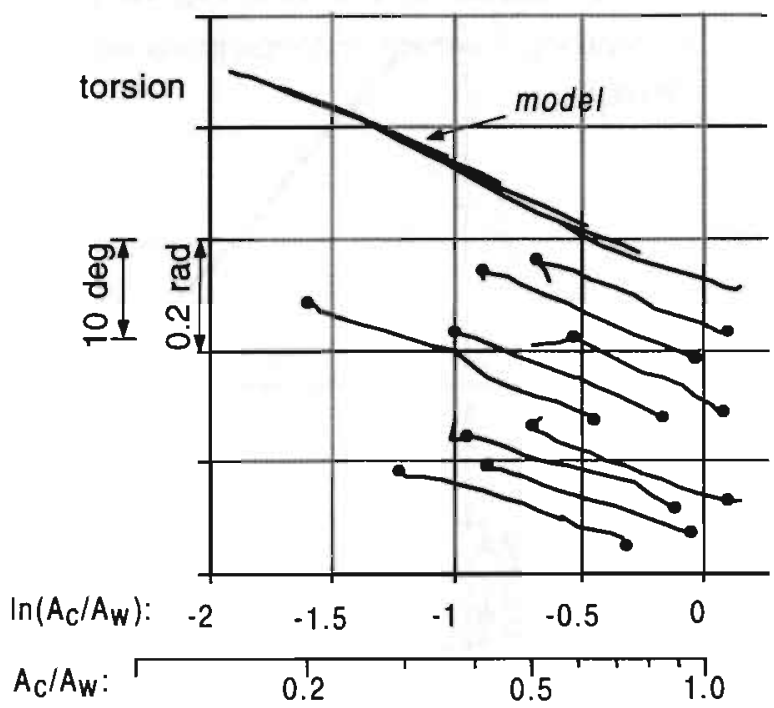

Figure 3.5 Relationship between torsion and area ejection during systole as measured by means of MRI, and according to a mathematical model of left ventricular mechanics. Area ejection was expressed as the logarithm of the ratio of cavity area $\left(A_{c}\right)$ to wall area $\left(A_{w}\right)$. Torsion was represented by the shear angle on the epicardium. The curves obtained from the volunteers are marked by dots, indicating the beginning and end of the ejection phase. The other curves are a result of simulations.

Figure 3.5 shows changes of torsion as a function of the logarithm of normalized cavity area for the 9 volunteers. For the purpose of proper presentation the curves are shifted arbitrarily along the torsion axis. During the ejection phase the relation between torsion and the logarithm of normalized cross-sectional cavity area was practically linear. Regression analysis, applied to the parts of the curves associated with the ejection phase, rendered a slope of $-0.173 \pm 0.024 \mathrm{rad}$.

In figure 3.5 also five simulations are shown of torsion as a function of area ejection, each curve having a different end-diastolic cavity volume. The ratio of cavity area to wall area at the beginning of the ejection phase ranged from 0.36 to 1.15 , and at the end of ejection from 0.15 to 0.57 . The mathematically predicted curves were similar to the measured curves. Linear regression, applied to the simulations, rendered a slope of $-0.194 \pm 0.026 \mathrm{rad}$. 


\subsection{DISCUSSION}

In healthy volunteers we found a systematic relationship between torsion and the ratio of cavity to wall cross-sectional area $\left(A_{c} / A_{*}\right)$. Applying linear regression to torsion as a function of $\ln \left(A_{c} / A_{w}\right)$ a slope was found of $-0.173 \pm 0.024 \mathrm{rad}$ (mean $\pm \mathrm{sd}$ ). In a mathematical model of LV wall mechanics this slope was predicted to be $0.194 \pm 0.026 \mathrm{rad}$. The slopes in the measurements and the simulations are so close that the basic assumptions made in the model are considered to be realistic approximations.

Cardiac disorders such as myocardial ischemia or infarction, and hypertrophic cardiomyopathy are known to influence the pattern of myocardial deformation (Prinzen et al., 1989; Maier et al., 1992; Young et al., 1994; Lima et al., 1995). Therefore, a method detecting these patterns has a potential clinical use. For example, in subendocardial ischemia the subendocardial fibers will, due to loss of function, shorten less than the subepicardial fibers. This results in an increase in torsion (Prinzen et al., 1984), what can be understood as follows. In the healthy heart the fibers in the inner and outer layers of the wall exert opposite torques. Torques due to the outer layers are larger than torques due to the inner layers because of the longer lever. This causes torsion to occur in favor of the outer layers. During subendocardial ischemia the counteracting torque of the inner layers is diminished and therefore torsion will increase. Added to the reduced fiber shortening during ischemia, the absolute value of the slope of the relation between torsion and area ejection is expected to increase. Furthermore, a kinematic model appears to be a useful tool in describing myocardial deformation. Once the normal deformation of the healthy heart has been accurately described, residuals from this normal pattern give information about pathology. Furthermore, these residuals can be described in the kinematic model by adding more parameters. In this way foreknowledge about regular cardiac motion can be used to enhance sensitivity in detecting motion disorders.

Assessment of the relation between dimensionless representations of torsion and contraction has received little attention in literature. In several reports torsion and contraction were investigated separately (Ohayon and Chadwick, 1988; Ingels et al., 1989; Hansen et al., 1991; Rademakers et al., 1992; Gibbons Kroeker et al., 1993; Buchalter et al., 1994; Young et al., 1994). Arts et. al. (Arts et al., 1984) measured the 
relation between torsion and contraction echographically in dogs. Furthermore, they compared their results with the predictions of a mathematical model of LV mechanics. Contraction was described by shortening of the inner circumference of the LV. The mathematical model predicted an approximately linear relationship between torsion and inner circumference shortening, with a slope of $2.37 \pm 0.36 \mathrm{rad}^{-1}$. Echographically a slope of $2.31 \pm 0.23 \mathrm{rad}^{-1}$ (mean \pm sd) was determined in a series of 11 measurements, each based on frame-to-frame analysis of 15 cardiac cycles. On the basis of the data in the present MR experiments we calculated a slope of $2.63 \pm 0.37$ $\mathrm{rad}^{-1}$ for the nine volunteers.

The ejection fraction (EF) is a clinical parameter which is widely used to characterize cardiac function. Although determination of the ejection fraction was not our primary goal, an estimate can be given using the cross-sectional area changes we determined (Appendix B):

$$
\mathrm{EF}=100 \%\left(1-\frac{A_{c, e c} / A_{w, e e}}{A_{c, b e} / A_{w, b e}}\right),
$$

with be and ee denoting the beginning and end of the ejection phase. The average ejection fraction thus obtained was $56.4 \pm 6.1 \%$ for the 9 volunteers, which is similar to values reported in literature (Semelka et al., 1990).

The accuracy of visual estimation of inner and outer radii of an LV cross-section is of the order of $\pm 1 \mathrm{~mm}$. Changes in the radii between two consecutive images are often less than $1 \mathrm{~mm}$. We estimated the radii visually at only one reference time. Using these estimates, changes in the radii were determined based on tag motion with the help of a kinematic model. Thus found inner and outer radii at the other times were within the range of accuracy of visual estimation at those times. Errors of $\pm 1 \mathrm{~mm}$ in the visually estimated radii at the reference time introduced errors of approximately $10 \%$ in the slope of the linearized relation between torsion and area ejection.

We acquired images only from short-axis cross-sections of the LV, so no information was obtained about motion in the long-axis direction. The assumption was made in the kinematic model that shortening of base to apex segment length is $65 \%$ of outer circumference shortening. This value is based on animal experiments (Feigl and Fry, 
1964). To compare, if no axial shortening is assumed in the kinematic model the slope of the relation between torsion and area ejection decreases by about $12 \%$. Since in the healthy heart axial shortening does occur, the systematic error in the slope due to our assumption is not expected to be larger than about $5 \%$.

Short-axis images of the LV were acquired from two fixed planes. Due to rigid body motion and shortening of the LV in the long-axis direction the cross-sections of the LV that were visualized changed in time. The myocardial tissue was tagged by selectively saturating planes orthogonal to the image plane. This was done in two perpendicular directions resulting in more or less cylindrical regions of unsaturated tissue orthogonal to the image plane, here called tag lines. The intersection of such a tag line with the image plane determines the location of a tag in the image. Because of rigid body motion and deformation of the LV the intersection of a tag line with the image plane changes in time. In this respect we made the assumption that in the equatorial region area ejection as well as torsion were independent of long-axis position (Buchalter et al., 1990). As a result, in the analysis rigid body displacement of the LV does not influence the calculation of area ejection and torsion. Measurement of torsion is also not influenced by axial shortening, as shown below. Physically, torsion is represented by the tangent of the shear angle at the epicardial surface. At end-diastole the tags are aligned with the long-axis, perpendicular to both short-axis cross-sectional planes. The intersections of the tags with the short-axis planes are detected, and their position is measured. The distance between these planes is fixed. So, the tangent of the shear angle is measured directly, meaning that the used measure of torsion is not influenced by the occurrence of axial shortening.

It is concluded that in systole the relation between torsion and area ejection is common to all left ventricles in healthy humans. This relation appeared close to predictions made by a mathematical model of LV wall mechanics. Since equilibria of forces in the wall were essential to this model, in the real heart the same equilibria are likely to play an important role. 


\section{REFERENCES}

Arts, T., Meerbaum, S., Reneman, R. S., and Corday, E. (1984) Torsion of the Left Ventricle during the Ejection Phase in the Intact Dog. Cardiovasc Res 18, 183193.

Arts, T. and Reneman, R. S. (1989) Dynamics of left ventricular wall and mitral valve mechanics - a model study. J Biomechanics 22, 261-271.

Buchalter, M. B., Rademakers, F. E., Weiss, J. L., Rogers, W. J., Weisfeldt, M. L., and Shapiro, E. P. (1994) Rotational delormation of the canine left ventricle measured by magnetic resonance tagging: effects of catecholamines, ischaemia, and pacing. Cardiovasc Res 28, 629-635.

Buchalter, M. B., Weiss, J. L., Rogers, W. J., Zemouni, E. A., Weisfeldt, M. L., Beyar, R., and Shapiro, E. P. (1990) Noninvasive Quantification of Left Ventricular Rotational Deformation in Nomal Humans Using Magnetic Resonance Imaging Myocardial Tagging. Circulation 81, 1236-1244.

Clark, N. R., Reichek, N., Bergey, P., Hoffman, E. A., Brownson, D., Palmon, L., and Axel, L. (1991) Circumferential Myocardial Shortening in the Normal Human Left Ventricle - Assessment by Magnetic Resonance Imaging Using Spatial Modulation of Magnetization. Circulation 84, 67-74.

Feigl, E. O. and Fry, D. L. (1964) Intramural myocardial shear during the cardiac cycle. Circ Res 14, 536-540.

Gibbons Kroeker, C. A., Ter Keurs, H. E. D. J., Knudtson, M. L., Tyberg, J. V., and Beyar, R. (1993) An optical device to measure the dynamics of apex rotation of the left ventricle. Am J Physiol 265, H1444-H1449.

Hansen, D. E., Daughters, G. T., Alderman, E. L., Ingels, N. B., Stinson, E. B., and Miller, D. C. (1991) Effect of volume loading, pressure loading, and inotropic stimulation on left ventricular torsion in humans. Circulation 83, 1315-1326.

Ingels, N. B., Hansen, D. E., Daughters, G. T., Stinson, E. B., Alderman, E. L., and Miller, D. C. (1989) Relation between longitudinal, circumferential, and oblique shortening and torsional deformation in the left ventricle of the transplanted human heart. Circ Res 64, 915-927.

Lima, J. A. C., Ferrari, V. A., Reichek, N., Kramer, C. M., Palmon, L., Llaneras, M. R., Tallant, B., Young, A. A., and Axel, L. (1995) Segmental motion and deformation of transmurally infarcted myocardium in acute postinfarct period. Am J Physiol 268, H1304-H1312.

Maier, S. E., Fischer, S. E., McKinnon, G. C., Hess, O. M., Krayenbuehl, H. P., and Boesiger, P. (1992) Evaluation of Left Ventricular Segmental Wall Motion in Hypertrophic Cardiomyopathy with Myocardial Tagging. Circulation 86, 1919-1928.

Muiitjens, A. M. M., Roos, J. M. A., Arts, T., Hasman, A., and Reneman, R. S. (1993) Extrapolation of Incomplete Marker Tracks by Lower Rank Approximation. Int J Biomed Comput 33, 219-239.

Ohayon, J. and Chadwick, R. S. (1988) Theoretical analysis of the effects of a radial activation wave and twisting 
motion on the mechanics of the left ventricle. Biomeology 25, 435-447.

Prinzen, F. W., Arts, T., Hoeks, A. P. G., and Reneman, R. S. (1989) Discrepancies between Myocardial Blood Flow and Fiber Shortening in the Ischemic Border Zone as assessed with Video Mapping of Epicardial Deformation. European $J$ of Physiology 415, 220-229.

Prinzen, F. W., Arts, T., Van der Vusse, G. J., and Reneman, R. S. (1984) Fiber shortening in the inner layers of the left ventricular wall as assessed from epicardial deformation during normoxia and ischemia. $J$ Biomechanics 17, 801811.

Rademakers, F. E., Buchalter, M. B., Rogers, W. J., Zerhouni, E. A., Weisfeldt, M. L., Weiss, J. L., and Shapiro, E. P. (1992) Dissociation between left ventricular untwisting and filling - Accentuation by catecholamines. Circulation 85, 1572 1581.
Semelka, R. C., Tomei, E., Wagner, S., Mayo, J., Kondo, C., Suzuki, J.-I., Caputo, G. R., and Higgins, C. B. (1990) Normal left ventricular dimensions and function: Interstudy reproducibility of measurements with cine MR imaging. Radiology 174, 763-768.

Young, A. A., Imai, H., Chang, C. N., and Axel, L. (1994) Two-dimensional Left Ventricular Deformation during Systole using Magnetic Resonance Imaging with Spatial Modulation of Magnetization. Circulation 89, 740-752.

Young, A. A., Kramer, C. M., Ferrani, V. A., Axel, L., and Reichek, N. (1994) Threedimensional left ventricular deformation in hypertrophic cardiomyopathy. Circulation 90, 854-867.

Zerhouni, E. A., Parish, D. M., Rogers, W. J., Yang, A., and Shapiro, E. A. (1988) Human Heart: Tagging with MR Imaging - A Method for Noninvasive Assessment of Myocardial Motion. Radiology 169, 59-63.

\section{APPENDIX A}

During ventricular contraction wall volume is approximately constant. Thus:

$$
\left(R_{o, t}{ }^{2}-r_{t}^{2}\right) H_{t}=\left(R_{o, r e j}{ }^{2}-r_{r e f}{ }^{2}\right) H_{r e f},
$$

with $R_{o}$ the outer radius of the LV cross-section, $r$ the distance of a point in the myocardial wall to the center of the LV cross-section, $H$ the length of a base to apex segment, and $t$ and ref denoting time $t$ and a reference time, respectively. Assuming logarithmic shortening of base to apex segment length to be $65 \%$ of logarithmic shortening of the outer circumference $\left(\ln \left(H_{t} / H_{, .,}\right)=0.65 \cdot \ln \left(R_{o, t} / R_{o, r r t}\right)\right)$, it follows that 


$$
r_{i}=\sqrt{R_{r, l}^{2}-\left(R_{r, r e f j}^{2}-r_{r e f}^{2}\right)\left(\frac{R_{n, r r f}}{R_{t}}\right)^{0.05}}
$$

Besides circumferential shortening, the LV cross-section undergoes rigid body rotation $(\Phi)$ and displacement $(\Delta \mathbf{M})$ with respect to the center of the cross-section at the reference time $\left(\mathbf{M}_{r e f}\right)$. Successive application of the preceding circumferential shortening, rotation, and displacement to a point in the myocardial wall gives equation (1). Equation (4) is obtained by substituting the inner radius of the LV cross-section $\left(R_{i}\right)$ for $r_{i}$ in equation (A2).

\section{APPENDIX B}

Because we only acquired images from short-axis cross-sections we cannot determine the ejected volume fraction (EF) directly. An estimate of EF can be obtained by assuming that the shape of the left ventricle is ellipsoidal, and that the cross-sectional area changes are measured at the equator. Then it holds that

$$
\frac{V_{c, e e}}{V_{c, b e}}=\frac{\frac{4}{3} \pi R_{i, e e}{ }^{2} H_{e e}}{\frac{4}{3} \pi R_{i, b e}{ }^{2} H_{b e}}=\frac{A_{c, e e} H_{e e}}{A_{c, b e} H_{b e}}=\frac{A_{c, e e} H_{e e}}{A_{c, b e} H_{b e}} \frac{A_{w, b e} H_{b e}}{A_{w, e c} H_{e e}}=\frac{A_{c, e e} / A_{w, e e}}{A_{c, b e} / A_{w, b e}},
$$

with $V_{c}$ representing the left ventricular cavity volume, $R_{i}$ the short-axis length of the cavity, and $H$ the long-axis length. The symbols $A_{c}$ and $A_{w}$ refer to the crosssectional cavity and wall areas respectively, and $b e$ and $e e$ denote the beginning and end of the ejection phase. Wall volume was assumed to be constant during ejection so that $A_{w} H$ remains constant. Then for the ejection fraction (EF), equation (5) follows. Note that long-axis shortening is implicitly accounted for in this approach, which is reflected in the different values for cross-sectional wall area for the beginning and end of ejection. 


\title{
Chapter 4
}

Quantification of 3D left ventricular deformation with a kinematic model using Magnetic Resonance cardiac tagging

\author{
F.W.L. Aelen', T. Arts ${ }^{1}$, D.G.M. Sanders ${ }^{3}$, F.W. Prinzen ${ }^{2}$, G.R.P. Thelissen ${ }^{3}$, \\ R. S. Reneman ${ }^{2}$
}

Departments of Biophysics ${ }^{1}$ and Physiology ${ }^{2}$, Cardiovascular Research Institute Maastricht, Maastricht University, PO Box 616, 6200 MD Maastricht, The Netherlands,

Department of Diagnostic Radiology ${ }^{3}$, University Hospital Maastricht, PO Box 5800 , 6202 AZ Maastricht, The Netherlands.

Submitted 


\subsection{ABSTRACT}

Cardiac wall deformation can be assessed using the Magnetic Resonance tagging technique. Conventionally, in each sequential image all tags and contours have to be identified, requiring much user-interaction. Furthermore, 2D displacement data from multiple cross-sections have to be combined to quantify 3D deformation. We present a method to determine the 3D motion field directly from the set of 2D images, thus bypassing the detection of the individual tags. MR-tagged images were acquired from two short-axis and two long-axis cross-sections. Left ventricular contours were delineated only at one reference time. MR-tagged images of the different crosssections were then simultaneously deformed back to a reference state using a $3 D$ kinematic model. Parameters in the model were varied until the back deformed images fitted best with the reference images. The method was shown to work in healthy volunteers. 


\subsection{INTRODUCTION}

Quantification of the left ventricular deformation pattern is an important tool in the assessment of left ventricular function, especially in cardiac pathologies such as myocardial ischemia, cardiac infarction, and hypertrophic cardiomyopathy (Prinzen et al., 1989; Maier et al., 1992; Young et al., 1994; Lima et al., 1995). Therefore, a method to quantify this deformation may be helpful in diagnosing these diseases.

Previously, several imaging modalities have been used to quantify left ventricular deformation. For clinical purposes noninvasive techniques such as Magnetic Resonance Imaging (MRI) (McVeigh, 1996) and echocardiography (Mailloux et al., 1987) are preferred over invasive techniques, such as cine radiography of implanted radiopaque markers (Ingels et al., 1989). While echocardiography and cine MRI only determine wall thickening, with Magnetic Resonance cardiac tagging (Zerhouni et al., 1988; Axel and Dougherty, 1989; McVeigh, 1996) also 3D deformations within the myocardial wall can be quantified. With this technique a pattern of tags is attached noninvasively to the cardiac tissue by applying magnetic field gradients in conjunction with radio-frequency pulses. Generally the tags are applied at end-diastole, after which they move along with the tissue they were attached to. Deformation of the tagging pattern, as visualized in MR images (figure 4.1), reflects deformation of the underlying myocardial wall.

In most of the previous approaches, MR cardiac tagging was primarily used to quantify local deformation (Azhari et al., 1993; Kraitchman et al., 1995; O'Dell et al., 1995). This proved to be a fruitful approach when studying the mechanics of the left ventricular wall locally, for instance to distinguish healthy from diseased tissue (Kramer et al., 1993; Young et al., 1994; Azhari et al., 1995; Lima et al., 1995). Interpretation of the results for routine clinical application, however, is still difficult. Alternatively, global left ventricular deformation may be described by a limited number of kinematic parameters. Then complex post-processing can be avoided (Arts et al., 1992; Park et al., 1996; Aelen ot al., 1997). Regional disorders influence left ventricular deformation in a relatively large region (Prinzen et al., 1989; Rechavia et al., 1995; Kramer et al., 1996). A model describing the global motion of the left ventricle may be used to assess these disorders. The allowed modes of motion in this 


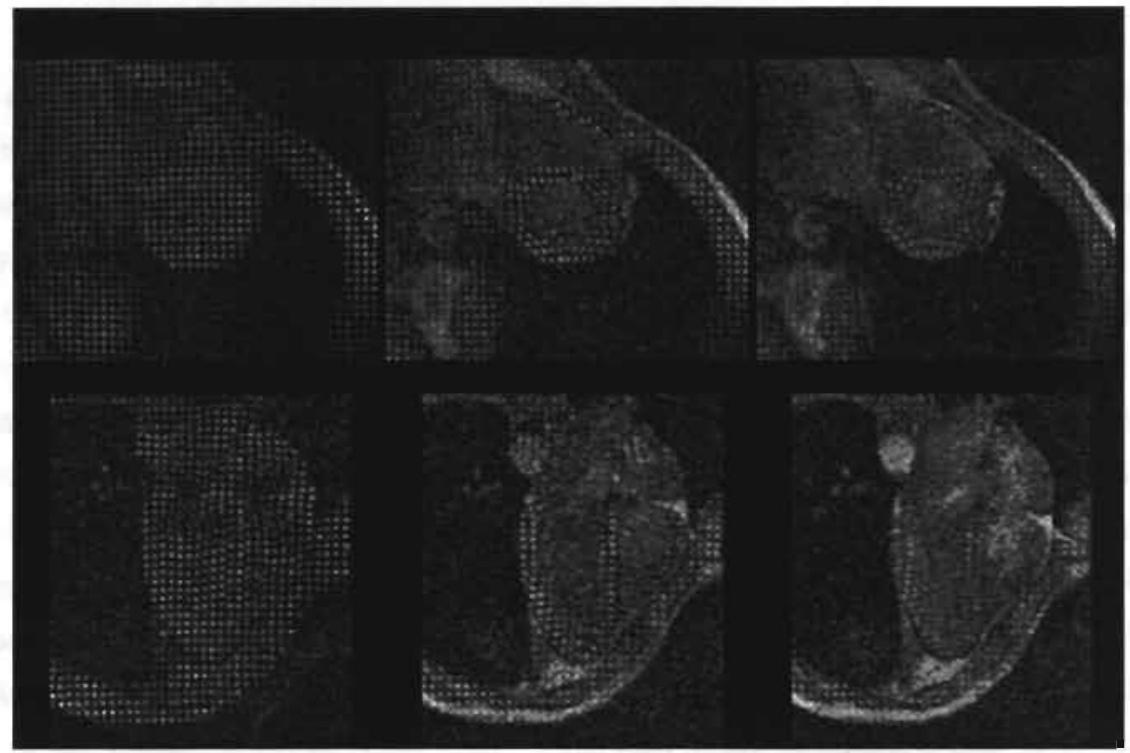

Figure 4.1 MR-tagged short-axis (above) and long-axis (below) images of the contracting cardiac left ventricle. The images were acquired 20 (left), 140 (middle), and $240 \mathrm{~ms}$ (right) after the R-wave of the ECG. The motion of the tags corresponds to myocardial deformation. Due to $T 1$-relaxation the tags slowly fade away, until after about $250 \mathrm{~ms}$ noise becomes dominant. In the left ventricular cavity the tags disappear shortly after application due to the ejection of blood.

model have to be chosen such that they reflect the characteristics of both the normal and the pathological components of motion.

Measurement of global left ventricular motion by means of MR cardiac tagging generally relies on following tags in the images and fitting their motion to a global kinematic model (O'Dell et al., 1995; Park et al., 1996; Aelen et al., 1997). Delineation of endocardial and epicardial contours in all images, necessary for most tag detection algorithms (Guttman et al., 1994; Young et al., 1994), requires a large amount of user-interaction. Furthermore, many tag detection algorithms rely on a specific shape of the tags (Guttman et al., 1994), reducing the general applicability of the method. 
In this study we developed a method to quantify global left ventricular motion directly from the images, without the need to detect individual tags. The method is highly automated and, in addition, does not depend on the exact shape of the tags. Furthermore, the method is flexible as regards the number and locations of the crosssections to be imaged.

As a first step healthy volunteers were investigated. MR-tagged images were acquired from two short-axis and two long-axis cross-sections of the left ventricle. Subsequently the images were bandpass filtered. A kinematic model, based on the one described by Arts et al (Arts et al., 1992), was used to describe global left ventricular deformation. Parameters in the model represented the different modes of global deformation. The images acquired immediately after application of the tags served as reference. In each cross-section and at each moment in time the image was deformed back to the reference as good as possible using the 3D kinematic model. So, for each moment in time a set of kinematic parameter values was obtained by least squares fitting of the back deformed images to their respective references. These parameters express the global patterns of $3 \mathrm{D}$ left ventricular deformation.

\subsection{RELATION BETWEEN IMAGE INFORMATION AND 3D LEFT VENTRICULAR KINEMATICS}

At the start of an MR tagging pulse sequence a pattem of tags is applied. This pattern deforms and fades away in subsequent images. In reconstructing 3D motion from 2D MR-tagged images the relation between image information and motion information has to be described.

Consider a pattern of tags in the reference state in one of the cross-sections. In the 3D space a tag extends with about equal intensity along a line perpendicular to the plane of observation (figure 4.2). Due to cardiac motion the tag displaces in 3D, but is detected only in 2D. Consequently a point $A_{\text {ref }}$ in the plane of observation in the reference state moves out of this plane to $A_{t}$. In an opposite manner, the observed point $B_{r}$ was out of this plane $\left(B_{r e j}\right)$ in the reference state before. 


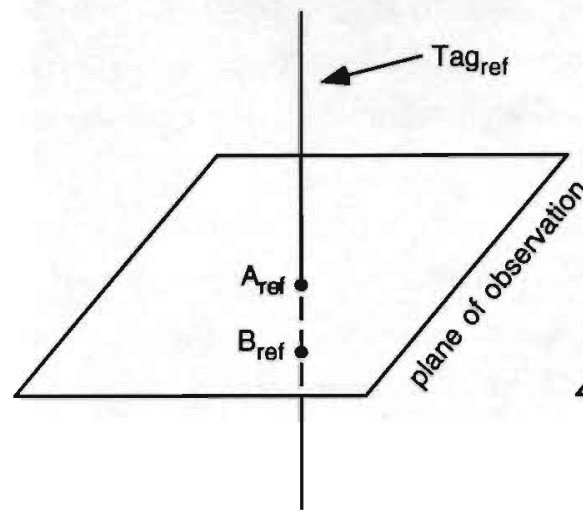

reference state

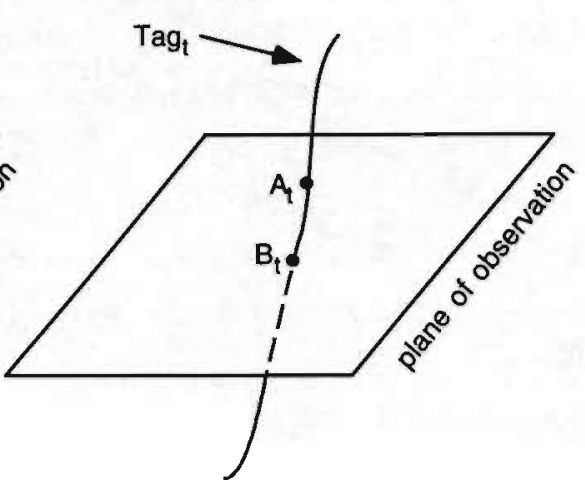

deformed state

Figure $4.2 \mathrm{~A}$ tag in the reference state (Tagret) extends in $3 D$ perpendicular to the plane of observation. The cross-section of the tag and this plane occurs in point $A_{\text {ret. }}$. After deformation, the tag (Tag) is observed in point $B_{t}$ the new cross-section of the tag with the plane of observation. The point $A_{\text {ref }}$ moved out of plane to position $A_{t}$. The point $B_{\text {ret }}$ is the position of $B_{\mathrm{f}}$ in the reference state, and is also out of plane.

The time of the reference state has been chosen as close as possible to the moment of application of the tags. At that time, the tags are straight and perpendicular to the plane of observation. In the present analysis we assumed that the tags still have these properties in the state used as reference. In figure 4.2 this implies that the points $A_{\text {ref }}$ and $B_{\text {ref }}$ have a common perpendicular line to the plane of observation. Note that the concept of tag in this respect applies to each pixel in the image.

\subsection{METHODS}

\subsubsection{Image acquisition and preprocessing}

Magnetic Resonance Imaging was performed on ten healthy volunteers using a 0.5 Tesla MR imaging system (Philips Gyroscan T5-II) with a body wrap-around coil. MRtagged images were acquired from two parallel short-axis cross-sections located at about 2 and $4 \mathrm{~cm}$ below the left ventricular base, and two perpendicular long-axis cross-sections (figure 4.1). A scan consisting of 16 images was acquired from each 
cross-section, starting $20 \mathrm{~ms}$ after the R-wave of the electrocardiogram (ECG) and with a time interval between the images of approximately $20 \mathrm{~ms}$. Prior to imaging a rectangular tagging grid was generated by Spatial Modulation of the Magnetization (SPAMM) (Axel and Dougherty, 1989) using two $90^{\circ}$ radio-frequency (RF) pulses for both orthogonal directions. The initial inter tag distance was $5 \mathrm{~mm}$ in both directions. T1-weighted MR imaging was utilized to acquire the images. The RF pulse flip angle for excitation was $40^{\circ}$. An echo time (TE) of $10 \mathrm{~ms}$ was used with a repetition time (TR) equal to the R-R interval in the ECG. The field of view was $213 \times 213 \mathrm{~mm}$ with a resolution of $256 \times 256$ pixels. Slice thickness was $8 \mathrm{~mm}$. Each scan was acquired in 5 to 6 minutes.

In addition to the tagged images, non-tagged T1-weighted MR gradient echo images were acquired from the same cross-sections. The non-tagged images at the reference time were used to manually delineate the cross-sections of the left ventricular wall. For the kinematic analysis left ventricular wall volume and cavity volume at the reference time were estimated from the cross-sections of the wall in both long-axis cross-sections. In this approach, the left ventricle was assumed to be rotationally symmetric around the long-axis, which was approximated in the images by a vertical line through the centers of gravity of the wall cross-sections. The contributions of both halves in both cross-sections to the volumes were averaged.

Due to T1 relaxation and tissue motion the tags in the MR-tagged images slowly faded away, until after about $250 \mathrm{~ms}$ noise became predominant. The contrast in the images was enhanced relative to the noise by matched spatial bandpass filtering. In the spatial frequency domain most of the information about the tags was contained within a square region defined by the inter tag distance. Spatial frequencies outside this region were diminished by multiplying the amplitude with a window function. This function is 1 at a square where either the horizontal or the vertical spatial frequency corresponds to the horizontal or vertical inter tag distance, respectively, and drops of cosine-like (Hanning window, (Press et al., 1988)) to zero at both sides of the square, being the center and the outside. 


\subsubsection{Kinematic Analysis}

A 3D kinematic model, based on the one employed by Arts et al (Arts et al., 1992), was used to describe the normal global pattern of systolic left ventricular deformation. A cardiac coordinate system, needed for a mathematical description of the deformation pattern, was defined as follows. The centers of gravity of the wall crosssections in the two short-axis reference images were determined. The origin of a fixed MR coordinate system was defined as the point in the middle between the centers of both cross-sections. Furthermore, the $x$ - and $y$-axes were chosen in the horizontal and vertical directions of the short-axis images, respectively, and the z-axis was chosen perpendicular to the $x$ - and $y$-axes, pointing towards the base of the left ventricle. The cardiac coordinate system was initially chosen to coincide with the MR coordinate system.

The modes of left ventricular motion described in the kinematic model were torsion $\left(k_{1}\right)$, cavity volume change $\left(k_{2}\right)$, ellipticalization $\left(k_{3}\right)$, and rigid body motion $\left(k_{4}-k_{9}\right)$. Torsion is associated with rotation of the apex with respect to the base and was quantified by the equatorial shear angle (Arts et al., 1992). A change in left ventricular cavity volume was described by an equal change of volume in two singular points, $\mathbf{H}_{1}$ and $\mathbf{H}_{2}$. Here, a singular point mathematically describes a spherically symmetric radial displacement towards it. So, cavity volume disappeared, i.e., was ejected, into the singular points. This was done in such a way that left ventricular wall volume remained constant. The two singular points were located on the long axis, $10 \mathrm{~mm}$ below and $10 \mathrm{~mm}$ above the cardiac origin, respectively. Furthermore, during the contraction the cardiac origin was allowed to move along the $z$-axis $\left(k_{10}\right)$ relative to the MR origin. The parameter $k_{2}$ was defined as cavity volume change normalized to wall volume $\left(V_{w}\right)$. Ellipticalization $\left(k_{3}\right)$ reflects a combination of circumferential contraction and longitudinal extension. Rigid body motion was described by rotation $\left(k_{4}-k_{6}\right)$ around, and translation $\left(k_{7}-k_{9}\right)$ along the three cardiac coordinate axes. The detailed mathematical description of the kinematic model is given in Appendix A. Using the kinematic model the motion is described of an arbitrary point at location $\mathbf{x}_{r f f}$ in the wall to the location $\mathbf{x}_{\text {def }}\left(\mathbf{x}_{\text {ref }}, k_{1}-k_{10}\right)$ in the deformed state (Appendix A).

The gray values of the MR-tagged images were transformed back to images in the reference state. In the reference state, the $x$ and $y$-coordinates coincided with the 
centers of the pixels. In each non-reference image the considered points coincided with the plane of cross-sectioning. The back transformation was performed iteratively as follows. Each pixel position $\mathbf{p}_{\text {ref }}$ in the reference state was transformed with equation (1) to a position $\mathbf{p}_{\imath}$ in the non-reference image. The point $\mathbf{p}_{\mathrm{r}}$ generally moved out of plane by a perpendicular distance $z$. This distance $z$ was subtracted from the position $\mathbf{p}_{r e f}$. The procedure was repeated until the distance $z$ became smaller than $0.1 \mathrm{~mm}$. The gray value of $\mathbf{p}$, was found by bilinear interpolation of the gray values of nearby pixels at integer positions. This gray value was attributed to the position of $\mathbf{p}_{r e f}$.

The accuracy of gray value interpolation was improved by doubling the pixel resolution of the acquired images before interpolation. This doubling was performed by Fast Fourier Transformation of the image to the frequency domain. For the high frequencies, zero values were added to the spectrum. Then the enlarged spatial frequency spectrum was transformed back to an image with double pixel resolution.

To limit computing time, the displacements of the points in the left ventricular crosssections were calculated by the kinematic model with a limited resolution of 3 pixels, corresponding to $2.5 \mathrm{~mm}$. Motion of the other points in between was calculated by means of bilinear interpolation. Near the endocardial and epicardial borders this bilinear interpolation could not be applied. There the kinematic model was used to calculate the displacements for all pixels.

The deformed images of the four cross-sections were simultaneously compared with their respective reference images by evaluating the following objective function $(F)$ :

$$
F(\mathbf{k})=\sqrt{\frac{1}{N-1} \sum_{j=1}^{4} \sum_{i=1}^{N_{j}}\left|I_{\text {defomed }}(\mathbf{k})-I_{\substack{\text { reference } \\ \text { inage }}}\right|^{2}},
$$

with $\mathbf{k}$ the kinematic parameters, $j$ indicating the left ventricular cross-section, $i$ the pixel in the cross-section, $N_{j}$ the total number of pixels in cross-section $j$, and $I$ the pixel gray value. A best fit between the deformed images and the reference images was found by minimizing the objective function by variation of the kinematic parameter values following Powell's method (Press et al., 1988). 


\subsection{RESULTS}

Representative bandpass filtered images from a short-axis and a long-axis crosssection are shown in figure 4.3 (left). This figure (right) also shows the images obtained by deforming the bandpass filtered images back to their reference states by means of the 3D kinematic model. Clearly, the deformed images resemble the initial rectangular tagging grid. Only close to the singular points the fit was not as good.

Kinematic parameter values were obtained during 12 time frames, representing the time interval from 20 to $240 \mathrm{~ms}$ after the R-wave in the ECG. For a representative volunteer the time courses of the estimated kinematic parameter values are shown in figure 4.4. Maximum translation of the left ventricle in the $x-, y-$, and $z$-direction was $1.55 \pm 0.49 \mathrm{~mm}, 1.70 \pm 1.01 \mathrm{~mm}$, and $6.25 \pm 0.92 \mathrm{~mm}$, respectively (mean \pm standard deviation, 10 volunteers). During the investigated time interval long-axis translation was directed towards the apex for all volunteers. Maximum rotation around the $x-, y-$, and $z$-axis was $0.074 \pm 0.036 \mathrm{rad}, 0.076 \pm 0.035 \mathrm{rad}$, and $0.089 \pm 0.033 \mathrm{rad}$, respectively. In all volunteers rigid body rotation around the long-axis was counterclockwise (upward slope) in early systole and reversed to clockwise (downward slope) during mid-systole, as viewed from apex to base. During systole, torsion increased by $0.105 \pm 0.028 \mathrm{rad}$. The ratio of cavity volume to wall volume decreased from $0.85 \pm 0.13$ to $0.47 \pm 0.12$. The fraction of cavity volume that was ejected during the 12 time frames was $45.2 \pm 7.1 \%$. For most volunteers the end of the ejection phase had not yet been reached at the last analyzed time frame. Note that during systole the time courses of torsion and normalized cavity volume change were approximately linear. Ellipticalization ranged from $-0.021 \pm 0.015$ to $0.037 \pm 0.025$, respectively. Time courses of the ellipticalization parameter did not show a distinct pattern for the ten volunteers. During systole the location of the cardiac origin along the long-axis moved $11.0 \pm 8.6$ $\mathrm{mm}$ towards the base. One volunteer showed a downward motion of the origin during systole.

The estimated parameter values depended on the position and orientation of the applied cardiac coordinate system. The position of the cardiac origin along the long axis was estimated by the kinematic model. Shifting this position by the expected inaccuracy of $5 \mathrm{~mm}$ perpendicular to the long-axis direction resulted in changes in parameter values on the order of $10 \%$. 


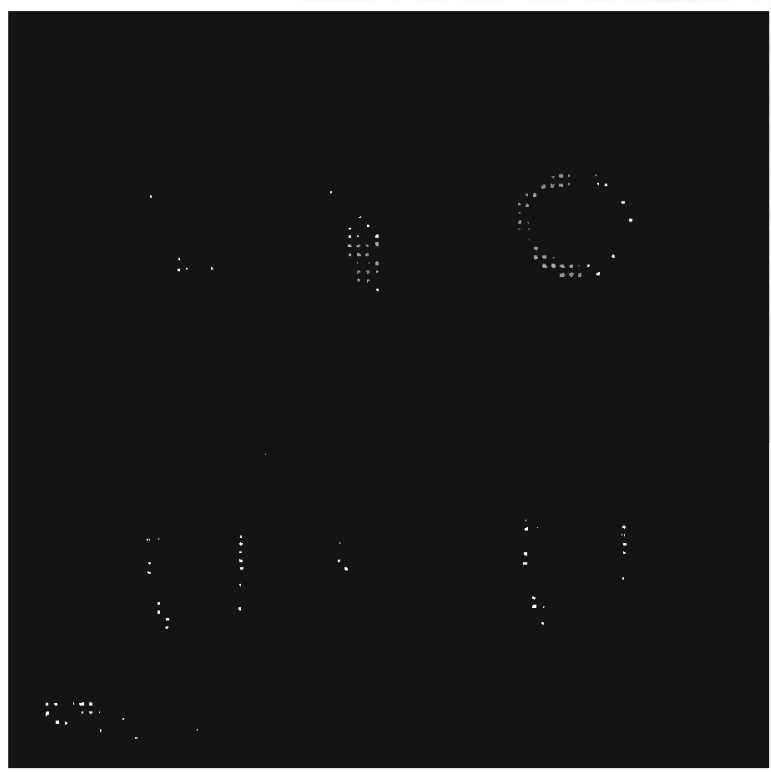

Figure 4.3 Left: Bandpass filtered short-axis (above) and long-axis (below) images, $140 \mathrm{~ms}$ after the R-wave of the ECG. Filtering enhanced the contrast in the images and made them more suitable for gray value interpolation. Right: Short-axis (above) and long-axis (below) images, $140 \mathrm{~ms}$ after the R-wave of the ECG, after deformation back to the reference state using a kinematic model of $3 D$ left ventricular deformation. Parameters in the model, representing the different modes of global deformation, were varied until the deformed images fitted best with the reference images, i.e., the images immediately after application of the tags (figure 4.1, middle).

Analysis time on a Pentium $133 \mathrm{MHz}$ was approximately 10 minutes for the manual delineation of endocardial and epicardial contours in the four reference images, and approximately one hour for the fully automated kinematic analysis.

\subsection{DISCUSSION}

A method was developed to quantify global systolic left ventricular deformation by means of MR cardiac tagging and a 3D kinematic model. Parameters describing the different modes of global motion were determined directly from the images, without 

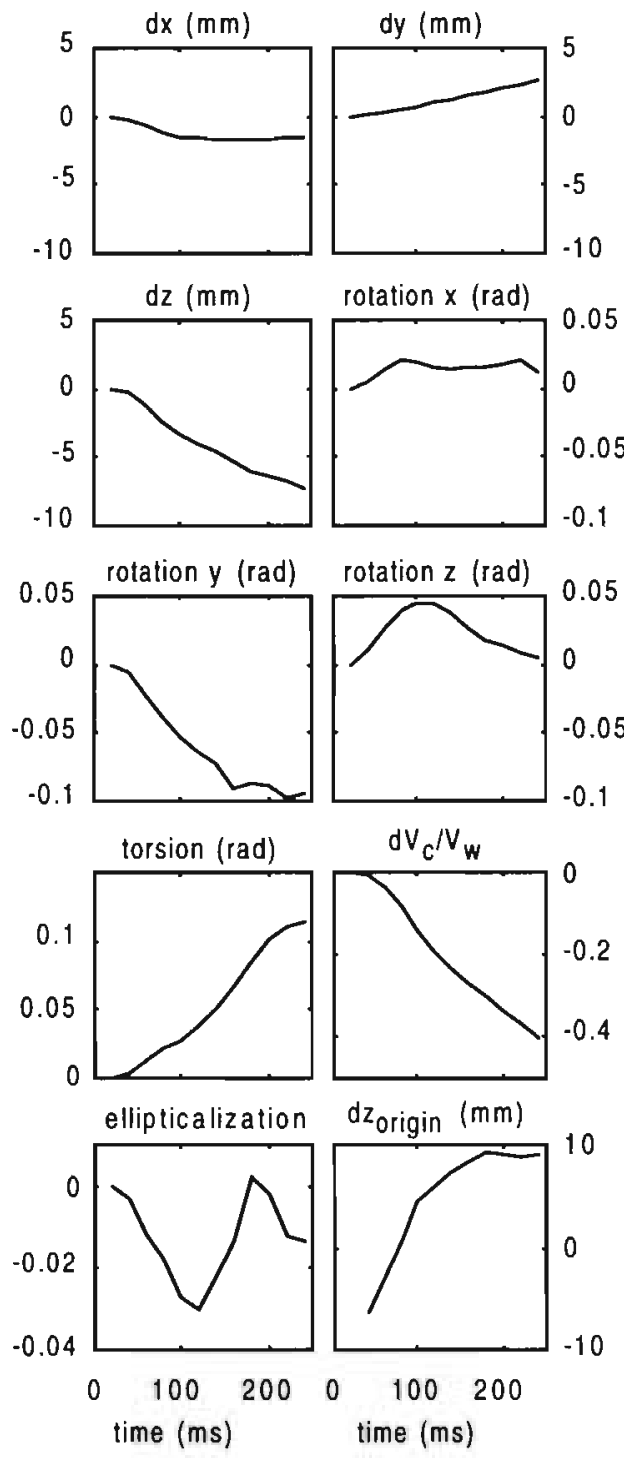

Figure 4.4 Kinematic parameter values of a representative volunteer as a function of time. Time zero refers to the $R$-wave in the ECG. Parameters used are rigid body translation (dx, $d y, d z)$ along and rotation around the $x$-, $y$-, and z-axes, torsion, cavity volume change normalized to wall volume $\left(d V_{\mathcal{C}} N_{w}\right)$, ellipticalization, and translation of the origin of the coordinate system relative to the left ventricle ( $d z_{\text {ongin }}$ ) along the long-axis. 
the need for detection of individual tags. The method is flexible with regard to the number, the location, and the direction of left ventricular cross-sections to be imaged. User interaction is limited to manual delineation of the left ventricular contours in the first image of each cross-section. It is demonstrated that the method works in healthy volunteers. In principle, the method can be used to study motion disorders for cardiac pathologies. For this purpose, parameters describing the specific aberrations from the normal deformation pattern due to these pathologies will have to be added to the kinematic model.

The kinematic parameter values found in this study were all within the normal range reported in literature. The left ventricle was found to rotate around the long-axis counterclockwise in early systole and clockwise during the second part of systole, as viewed from apex to base. This is in agreement with the findings of Qi et al (Qi et al., 1993) and Young et al (Young et al., 1994). Relatively large values for the standard deviations found for rotation and translation can be explained by the fact that rigid body motion is to a large extent determined by how the heart is placed in the chest, which is subject to relatively large biological variance. With regard to the deformation parameters, we found that a fraction of $45.247 .1 \%$ of the cavity volume was ejected. This is somewhat smaller than the ejection fraction values reported by Semelka et al (Semelka et al., 1990), which can be explained by the fact that for most volunteers not the entire ejection phase was covered. Torsion increased during systole by an amount of $0.105 \pm 0.028 \mathrm{rad}$, what is similar to the value of $0.087 \pm 0.010 \mathrm{rad}$ found by Buchalter et al (Buchalter et al., 1990). The practically linear relationship between torsion and the logarithm of the ratio of cavity volume to wall volume (figure 4.5) is in agreement with a previous study (Aelen et al., 1997), in which the motion was assessed from individually detected tags. Also the slope of the relation was very similar: $-0.178 \pm 0.037 \mathrm{rad}$ in the present study, as compared to $-0.173 \pm 0.024 \mathrm{rad}$ reported earlier (Aelen et al., 1997).

In order to obtain an impression of the reproducibility of the method, one volunteer was imaged twice, with 5 days in between the sessions. Maximum values of the deformation parameters were significantly different between the two sessions: 0.129 vs. $0.073 \mathrm{rad}$ for torsion, -0.461 vs. -0.338 for the change in the ratio of cavity volume to wall volume, and 0.046 vs. 0.075 for the ellipticalization. Apparently, there was a difference in the hemodynamic condition, which can be due to a number of 


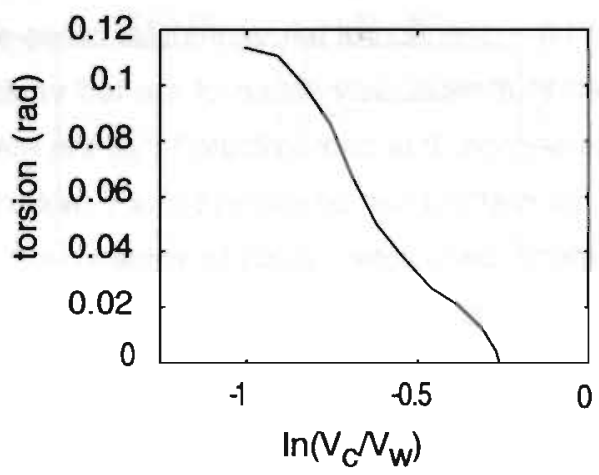

Figure 4.5 Torsion as a function of the logarithm of the ratio of cavity volume to wall volume for a representative volunteer. This relationship is practically linear, with a well defined slope, for all volunteers.

circumstances. However, the slope of the systolic relation between torsion and the logarithm of the ratio of cavity volume to wall volume, a more invariant measure (Arts et al., 1984), was found to be very similar for the two sessions: -0.219 vs. $-0.221 \mathrm{rad}$. This supports the reproducibility of the method.

Techniques used to determine local strains by means of MR cardiac tagging (Young et al., 1994; Azhari et al., 1995; Lima et al., 1995) have provided valuable information about local cardiac wall mechanics. There are, however, a number of difficulties associated with these techniques in relation to routine clinical application. For instance, they require a large number of cross-sections to be imaged in order to obtain a sufficiently high spatial resolution for the quantification of local strains. Moreover, the large number of local data collected has to be interpreted. It seems fruitful to investigate motion disorders of cardiac diseases using a kinematic model, in which foreknowledge about expected motion patterns can be used to enhance sensitivity in detecting the disorders. Regional disorders, such as for instance infarction, influence the pattern of deformation in a relatively large region of the left ventricle (Kramer et al., 1996). A limited number of parameters describing characteristic motion patterns will probably make interpretation of the results easier. To describe global 3D left ventricular deformation Park et al (Park et al., 1996) used a class of volumetric deformable models, the parameters of which were functions. They 
were able to assess quantitatively differences in motion between volunteers and patients with hypertrophic cardiomyopathy. In this approach, however, the motion of all individual tags had to be extracted prior to parameter estimation. Moreover, endocardial and epicardial contours had to be delineated in all images, requiring a large amount of user-interaction. Displacement data of the different cross-sections had to be combined in order to estimate local 3D displacements, after which global motion patterns were investigated. We propose a method to quantify the global 3D left ventricular motion patterns directly from the images, without the need to first determine local wall motion. The problem of through-plane motion was dealt with using image information from multiple, mutually perpendicular cross-sections. Left ventricular contours have to be delineated at only one reference time. Subsequent deformation of the contours is incorporated in the global motion analysis.

It should be noted that our method does not rely on tag detection algorithms, and can in principle also be used for analyzing cardiac wall deformation using more elaborate tagging schemes like CSPAMM (Fischer et al., 1993), breath-hold MRI (McVeigh and Atalar, 1992), or echo-planar imaging (Tang et al., 1995). Principally, there is no limitation to apply our method to line tagged images instead of images with a tagging grid. Tagging was only used to apply sufficient contrast in the images.

The kinematic model used to describe left ventricular deformation is a modification of the one used by Arts et al (Arts et al., 1992). The present model does not include the four modes of deformation used to describe asymmetric shape changes. These modes of deformation were found to be negligible for the healthy systolic heart. Another difference with the model by Arts et al was that we used two singular points instead of one to describe cavity volume change. When using only one singular point the fit was found to be less accurate in the long-axis cross-sections near the singular point, because in the kinematic model the nearby wall was pulled towards the singularity. This problem was reduced by adding a second singular point, such that the influence of the singularity was spread over a larger region. The distance of 20 $\mathrm{mm}$ between the two singularities was found to be optimal. Enlarging this distance slightly improved the global fit between back deformed and reference images but deteriorated the local fit near the apex considerably. Narrowing the distance between the singular points resulted in problems similar to the ones encountered when using only one singular point. Due to the use of two singular points values of the 
ellipticalization parameter cannot be compared directly with the values found by Arts et al (Arts et al., 1992).

The bandpass filter diminished the high and low spatial frequencies in the MR-tagged images, causing the loss of some information. In order to assess the possible loss of relevant information about deformation test images were generated. The 3D kinematic model was used to generate time sequences of images representing the two short-axis and the two long-axis cross-sections. At the first time point a rectangular sine modulated grid pattern of gray values was applied to the images. In the following images this pattern was deformed according to the kinematic model. Parameter values used in the kinematic model were all within the physiological range. Subsequently, the parameter values were re-estimated by means of our method. No significant differences were found between true and estimated parameter values, indicating that bandpass filtering did not introduce significant errors.

In conclusion, a method was developed to quantify global 3D left ventricular deformation patterns directly from MR-tagged images. The method is highly automated and no individual tags need to be detected. The method works in healthy volunteers and has the potential to be extended to assess more complicated modes of motion disorders which may occur due to disorders in cardiac contraction.

\section{REFERENCES}

Aelen, F. W. L., Arts, T., Sanders, D. G. M., Thelissen, G. R. P., Muijtjens, A. M. M., Prinzen, F. W., and Reneman, R. S. (1997) Relation between torsion and crosssectional area change in the human left ventricle. J Biomechanics 30, 207-212.

Arts, T., Hunter, W. C., Douglas, A., Muytjens, A. M. M., and Reneman, R. S. (1992) Description of the Deformation of the Left Ventricle by a Kinematic Model. $J$ Biomechanics 25, 1119-1127.

Arts, T., Meerbaum, S., Reneman, R. S., and Corday, E. (1984) Torsion of the Left
Ventricle during the Ejection Phase in the Intact Dog. Cardiovasc Res 18, 183-193.

Axel, L. and Dougherty, L. (1989) MR Imaging of Motion with Spatial Modulation of the Magnetization. Radiology 171, 841-845.

Azhari, H., Weiss, J. L., Rogers, W. J., Siu, C. O., and Shapiro, E. P. (1995) A noninvasive comparative study of myocardial strains in ischemic canine heart using tagged MRI in 3-D. Am J Physiol 268, H1918-H1926.

Azhari, H., Weiss, J. L., Rogers, W. J., Siu, C. O., Zerhouni, E. A., and Shapiro, E. P. 
(1993) Noninvasive Quantification of Principal Strains in Normal Canine Hearts using Tagged MRI images in 3-D. Am $J$ Physiol 264, H205-H216.

Buchalter, M. B., Weiss, J. L., Rogers, W. J., Zemouni, E. A., Weisfeldt, M. L., Beyar, R, and Shapiro, E. P. (1990) Noninvasive Quantification of Left Ventricular Rotational Deformation in Normal Humans Using Magnetic Resonance Imaging Myocardial Tagging. Circulation 81, 1236-1244.

Fischer, S. E., McKinnon, G. C., Majer, S. E., and Boesiger, P. (1993) Improved Myocardial Tagging Contrast. Magn Reson Med 30, 191-200.

Guttman, M. A., Prince, J. L., and McVeigh, E. R. (1994) Tag and contour detection in tagged MR images of the left ventricle. IEEE T Med Imaging 13, 74-88.

Ingels, N. B., Hansen, D. E., Daughters, G. T., Stinson, E. B., Alderman, E. L., and Miller, D. C. (1989) Relation between longitudinal, circumferential, and oblique shortening and torsional deformation in the left ventricle of the transplanted human heart. Circ Res 64, 915-927.

Kraitchman, D. L., Young, A. A., Chang, C.-N., and Axel, L. (1995) Semi-automatic tracking of myocardial motion in MR-tagged images. IEEE T Med Imaging 14, 422-433.

Kramer, C. M., Lima, J. A. C., Reichek, N., Ferrari, V. A., Llaneras, M. R., Palmon, L. C. Yeh, I.-T., Tallant, B., and Axel, L. (1993) Regional differences in function within noninfarct myocardium during left ventricular remodeling. Circulation $\mathbf{8 8}$, 1279-1288.

Kramer, C. M., Rogers, W. J., Theobald, T. M., Power, T. P., Petruolo, S., and Reichek, N. (1996) Remote noninfarcted region dysfunction soon after first anterior myocardial infarction. A Magnetic Resonance Tagging study. Circulation 94, 660-666.

Lima, J. A. C., Ferrari, V. A., Reichek, N., Kramer, C. M., Palmon, L., Llaneras, M. R., Tallant, B., Young, A. A., and Axel, L. (1995) Segmental motion and deformation of transmurally infarcted myocardium in acute postinfarct period. Am J Physiol 268, H1304-H1312.

Maier, S. E., Fischer, S. E., McKinnon, G. C., Hess, O. M., Krayenbuehl, H. P., and Boesiger, P. (1992) Evaluation of Left Ventricular Segmental Wall Motion in Hypertrophic Cardiomyopathy with Myocardial Tagging. Circulation 86, 19191928.

Mailloux, G. E., Bleau, A., Bertrand, M., and Petitclerc, R. (1987) Computer Analysis of Heart Motion from Two-dimensional Echocardiograms. IEEE T Bio Mod Eng 34, 356-364.

McVeigh, E. R. (1996) MRI of myocardial function: motion tracking techniques. Magn Reson Imaging 14, 137-150.

McVeigh, E. R. and Atalar, E. (1992) Cardiac Tagging with Breath-Hold Cine MRI. Magn Res Med 28, 318-327.

O'Dell, W. G., Moore, C. C., Hunter, W. C., Zerhouni, E. A., and McVeigh, E. R. (1995) Three-dimensional myocardial deformations: calculation with displacement field fitting to tagged MR images. Radiology 195, 829-835.

Park, J., Metaxas, D., and Axel, L. (1996) Analysis of left ventricular wall motion based on volumetric deformable models and MRI-SPAMM. Med Image Anal 1, 5371.

Press, W. H., Flannery, B. P., Teukolsky, S. A., and Vetterling, W. T. (1988) Numerical 
recipes - The art of scientific computing. Cambridge University Press, Cambridge.

Prinzen, F. W., Arts, T., Hoeks, A. P. G., and Reneman, R. S. (1989) Discrepancies between Myocardial Blood Flow and Fiber Shortening in the Ischemic Border Zone as assessed with Video Mapping of Epicardial Deformation. Eur J Physiol 415, 220-229.

Qi, P., Thomsen, C., Ståhlberg, F., and Henriksen, O. (1993) Normal Left Ventricular Wall Motion Measured with Two-dimensional Myocardial Tagging. Acta Radiol 34, 450-456.

Rechavia, E., De Silva, R., Nihoyannopoulos, P., Lammertsma, A. A., Jones, T., and Masen, A. (1995) Hyperdynamic performance of remote myocardium in acute infarction - Correlation between regional contractite function and myocardial perfusion. Eur Heart $J$ 16, 1845-1850.

Semelka, R. C., Tomei, E., Wagner, S., Mayo, J., Kondo, C., Suzuki, J.-l., Caputo, G. R., and Higgins, C. B. (1990) Normal left ventricular dimensions and function:
Interstudy reproducibility of measurements with cine MR imaging. Radiology 174, 763768.

Tang, C., McVeigh, E. R., and Zerhouni, E. A. (1995) Multi-shot EPI for improvement of myocardial tagging contrast: comparison with segmented SPGR. Magn Res Med 33, 443-447.

Young, A. A., Imai, H., Chang, C. N., and Axel, L. (1994) Two-dimensional Left Ventricular Deformation during Systole using Magnetic Resonance Imaging with Spatial Modulation of Magnetization. Circulation 89, 740-752.

Young, A. A., Kramer, C. M., Ferrari, V. A., Axel, L., and Reichek, N. (1994) Threedimensional left ventricular deformation in hypertrophic cardiomyopathy. Circulation 90, 854-867.

Zerhouni, E. A., Parish, D. M., Rogers, W. J., Yang, A., and Shapiro, E. A. (1988) Human Heart: Tagging with MR Imaging - A Method for Noninvasive Assessment of Myocardial Motion. Radiology 169, 59-63.

\section{Appendix A. kinematic model}

The modes of left ventricular motion described in the kinematic model were torsion $\left(k_{1}\right)$, cavity volume change $\left(k_{2}\right)$, ellipticalization $\left(k_{3}\right)$, and rigid body motion $\left(k_{4}-k_{9}\right)$. Rigid body motion was described by rotation $\left(k_{4}-k_{6}\right)$ around, and translation $\left(k_{7}-k_{9}\right)$ along the three cardiac coordinate axes. During contraction the cardiac origin was allowed to move along the z-axis $\left(k_{10}\right)$ relative to the MR origin. In a mathematical form, expressed in the MR coordinate system, a point $\mathbf{x}_{r e f}=\left(x_{r e f}, y_{r e f}, z_{r e f}\right)^{T}$ in the wall moves to the point $\mathbf{x}_{d e f}$ according to the thus defined kinematic model: 


$$
\begin{gathered}
\mathbf{x}_{\Delta e f}=\left[\begin{array}{c}
0 \\
0 \\
k_{50}
\end{array}\right]+\left[\begin{array}{l}
k_{7} \\
k_{8} \\
k_{9}
\end{array}\right]+\left[\begin{array}{ccc}
\cos \left(k_{6}\right) & -\sin \left(k_{6}\right) & 0 \\
\sin \left(k_{6}\right) & \cos \left(k_{6}\right) & 0 \\
0 & 0 & 1
\end{array}\right] \cdot\left[\begin{array}{ccc}
\cos \left(k_{5}\right) & 0 & \sin \left(k_{5}\right) \\
0 & 1 & 0 \\
-\sin \left(k_{5}\right) & 0 & \cos \left(k_{5}\right)
\end{array}\right] \\
{\left[\begin{array}{ccc}
1 & 0 & 0 \\
0 & \cos \left(k_{4}\right) & -\sin \left(k_{4}\right) \\
0 & \sin \left(k_{4}\right) & \cos \left(k_{4}\right)
\end{array}\right] \cdot\left[\begin{array}{ccc}
e^{-k_{5} / 3} & 0 & 0 \\
0 & e^{-k_{5} / 3} & 0 \\
0 & 0 & e^{2 k_{3} / 3}
\end{array}\right] \cdot \mathbf{x}_{3}}
\end{gathered}
$$

with: $\quad \mathbf{x}_{3}=\mathbf{H}_{2}+\sqrt[3]{1+3\left(k_{2} / 2\right) V_{w} / 4 \pi\left|\mathbf{x}_{2}-\mathbf{H}_{2}\right|^{3}} \cdot\left(\mathbf{x}_{2}-\mathbf{H}_{2}\right), \quad \mathbf{H}_{2}=\left[\begin{array}{c}0 \\ 0 \\ h_{2}\end{array}\right], h_{2}=10 \mathrm{~mm}$,

$$
\begin{aligned}
& \mathbf{x}_{2}=\mathbf{H}_{1}+\sqrt[3]{1+3\left(k_{2} / 2\right) V_{w} / 4 \pi\left|\mathbf{x}_{1}-\mathbf{H}_{1}\right|^{3}} \cdot\left(\mathbf{x}_{1}-\mathbf{H}_{1}\right), \quad \mathbf{H}_{1}=\left[\begin{array}{c}
0 \\
0 \\
h_{1}
\end{array}\right], h_{1}=-10 \mathrm{~mm}, \\
& \text { and, } \quad \mathbf{x}_{1}=\left[\begin{array}{ccc}
\cos \left(k_{1} z_{r e f} /\left|\mathbf{x}_{r e f}\right|\right) & -\sin \left(k_{1} z_{r e f} / \mathbf{x}_{r e f} \mid\right) & 0 \\
\sin \left(k_{1} z_{r e f} /\left|\mathbf{x}_{r e f}\right|\right) & \cos \left(k_{1} z_{r e f} /\left|\mathbf{x}_{r e f}\right|\right) & 0 \\
0 & 0 & 1
\end{array}\right] \cdot\left(\mathbf{x}_{r e f}-\left[\begin{array}{c}
0 \\
0 \\
k_{10}
\end{array}\right]\right),
\end{aligned}
$$

where $\mathbf{H}_{1}$ and $\mathbf{H}_{2}$ are the two singular points. Note that the parameter $k_{10}$, responsible for displacement of the location of the cardiac origin, occurs at two places in the equation. 


\section{Chapter 5}

Automated quantification of 3D deformation of the healthy and infarcted left ventricle using Magnetic Resonance cardiac tagging

F.W.L. Aelen ${ }^{1}$, T. Arts ${ }^{1}$, F.W. Prinzen ${ }^{2}$, G. Snoep ${ }^{3}$ and R.S. Reneman ${ }^{2}$

Departments of Biophysics ${ }^{1}$ and Physiology ${ }^{2}$, Cardiovascular Research Institute Maastricht, Maastricht University, PO Box 616, 6200 MD Maastricht, The Netherlands,

Department of Diagnostic Radiology ${ }^{3}$, University Hospital Maastricht, PO Box 5800 , 6202 AZ Maastricht, The Netherlands. 


\subsection{ABSTRACT}

Magnetic Resonance (MR) cardiac tagging is a non-invasive technique to measure left ventricular deformation. Tags are parts of the cardiac tissue in which the magnetization is altered locally. During the cardiac cycle the pattern of tags deforms along with the tissue. Current methods to analyze MR tagged images require a large amount of user-interaction, making them less suitable for routine clinical application. The goal of this study was to develop a highly automated method to quantify cardiac deformation from MR tagged images that is widely applicable. The method presented is based on back-deformation of the deformed tagging pattern to its initial, undeformed state. No individual tags needed to be detected. With minimal userinterference image information from multiple cross-sections was combined to reconstruct $3 \mathrm{D}$ wall motion. Wall thickening and circumferential, longitudinal, and area strains were determined in six healthy volunteers and two patients with myocardial infarction. Strain values found for the volunteers were within the normal physiological range. Furthermore, in the patients the infarcted region could be localized by detecting regional reductions in area strain. Manual interference time was approximately 10 minutes per subject. In conclusion, the method developed is able to quantify 3D deformation in inhomogeneously beating hearts in a highly automated way. 


\subsection{INTRODUCTION}

Cardiac abnormalities such as myocardial infarction and hypertrophic cardiomyopathy have been shown to influence the pattern of left ventricular deformation (Prinzen et al., 1989; Young et al., 1994; Lima et al., 1995; Marcus et al., 1997). In recent years, Magnetic Resonance cardiac tagging appeared to be a promising technique to diagnose cardiac diseases (Zerhouni et al., 1988; Axel and Dougherty, 1989; McVeigh, 1996). With this technique a pattern of tags can be attached noninvasively to the cardiac tissue by local alteration of tissue magnetization. The tagging pattern deforms along with the tissue (figure 5.1). Visualization of the tags in MR images acquired through the cardiac cycle provides information on motion and deformation of the cardiac walls.

Several studies have shown that contractile dysfunction can be assessed by quantitative analysis of tag motion (Kramer et al., 1993; Young et al., 1994; Azhari et al., 1995; Lima et al., 1995; Azhari et al., 1996). However, current methods to analyze MR tagged images are sub-optimal for several reasons. Tedious and time-consuming post-processing is needed, especially for manual or semi-automatic delineation of left ventricular contours, usually for all images (McVeigh, 1998). Tag detection is often highly automated but the method of detection depends on the shape of the tags, and therefore on the applied tagging procedure. Furthermore, after detection of the tags and contours information on $2 \mathrm{D}$ tag motion in perpendicular cross-sections has to be merged to obtain a 3D motion field throughout the left ventricle. Analysis methods with minimal user-interaction and high flexibility to handle images obtained with different tagging protocols would greatly enhance clinical applicability of MR cardiac tagging. Previously (chapter 4), we developed a highly automated method that was able to quantify the global 3D systolic motion patterns occurring in the normal left ventricle. In the present study this method was extended in order to quantify $3 D$ deformation in pathological hearts, i.e., hearts with inhomogeneous contraction pattems.

In the proposed method the heart is considered to be a 3D geometry which deforms as a function of time. The contours of cardiac geometry are delineated in a limited number of known cross-sections, imaged by regular MRI just after detection of the Rwave in the electrocardiogram (ECG). This set of contours is assembled to the $3 D$ 


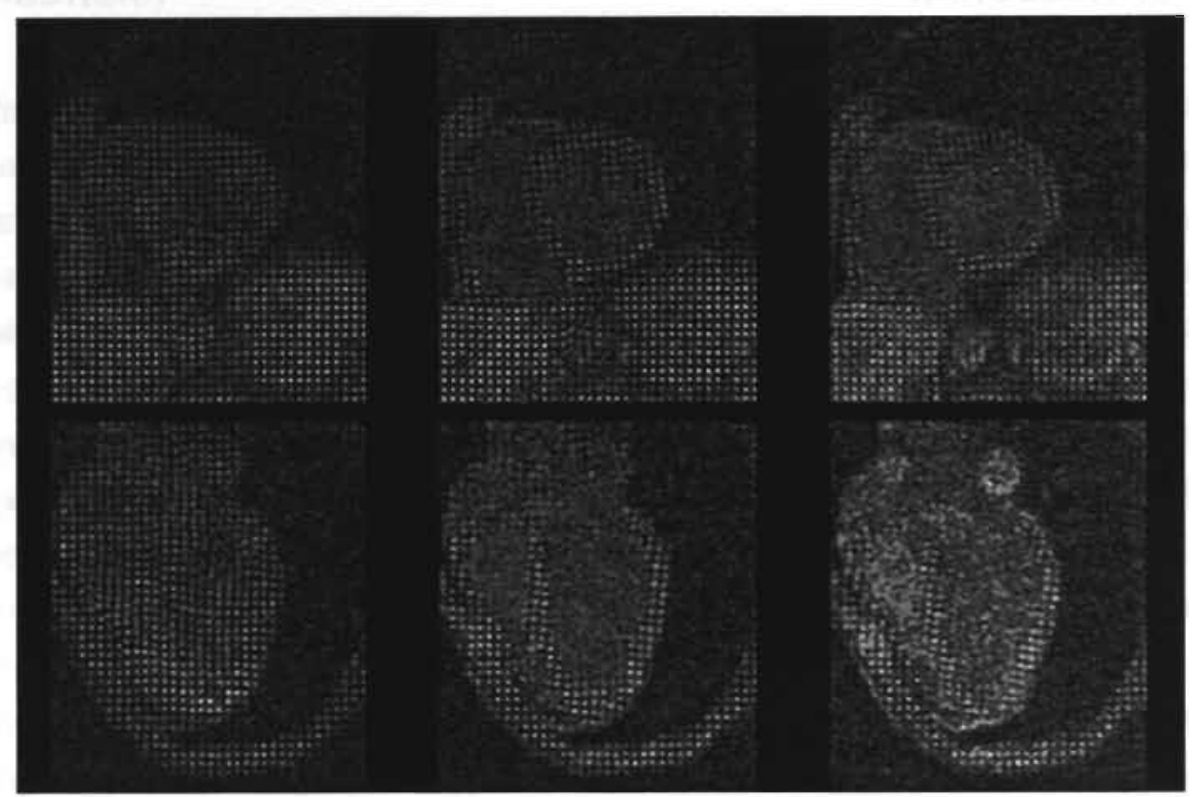

Figure 5.1 Representative MR tagged short-axis (above) and long-axis (below) images of the left ventricle, 20 (left), 80 (middle), and 140 (right) ms after the $R$-wave in the ECG. Motion of the tags corresponds to tissue motion. The tags in the left ventricular cavity disappear shortly after application of the grid due to blood flow.

cardiac geometry in the reference state. In a next acquisition series of MR images, tags are applied prior and as close as possible to reference time. At this time, in each imaged section the tags are visualized as a practically rectangular grid (figure 5.1; left). In a kinematic model 3D motion of each point in the cardiac wall is described by a set of parameters, which values vary as a function of time. Given these parameter values, tags in every imaged section can be deformed back to the images in the reference state. In the analysis, the parameter values are adjusted so that the thus back-deformed images with tags correlate best with the images at the reference time, having a practically rectangular grid of tags (figure 5.2). An important advantage is that no individual tags need to be detected.

The present study was meant to show the feasibility of the above-described approach to quantify local 3D left ventricular wall motion. To this purpose, MR tagged images were acquired only from two short-axis and two long-axis cross-sections during the 


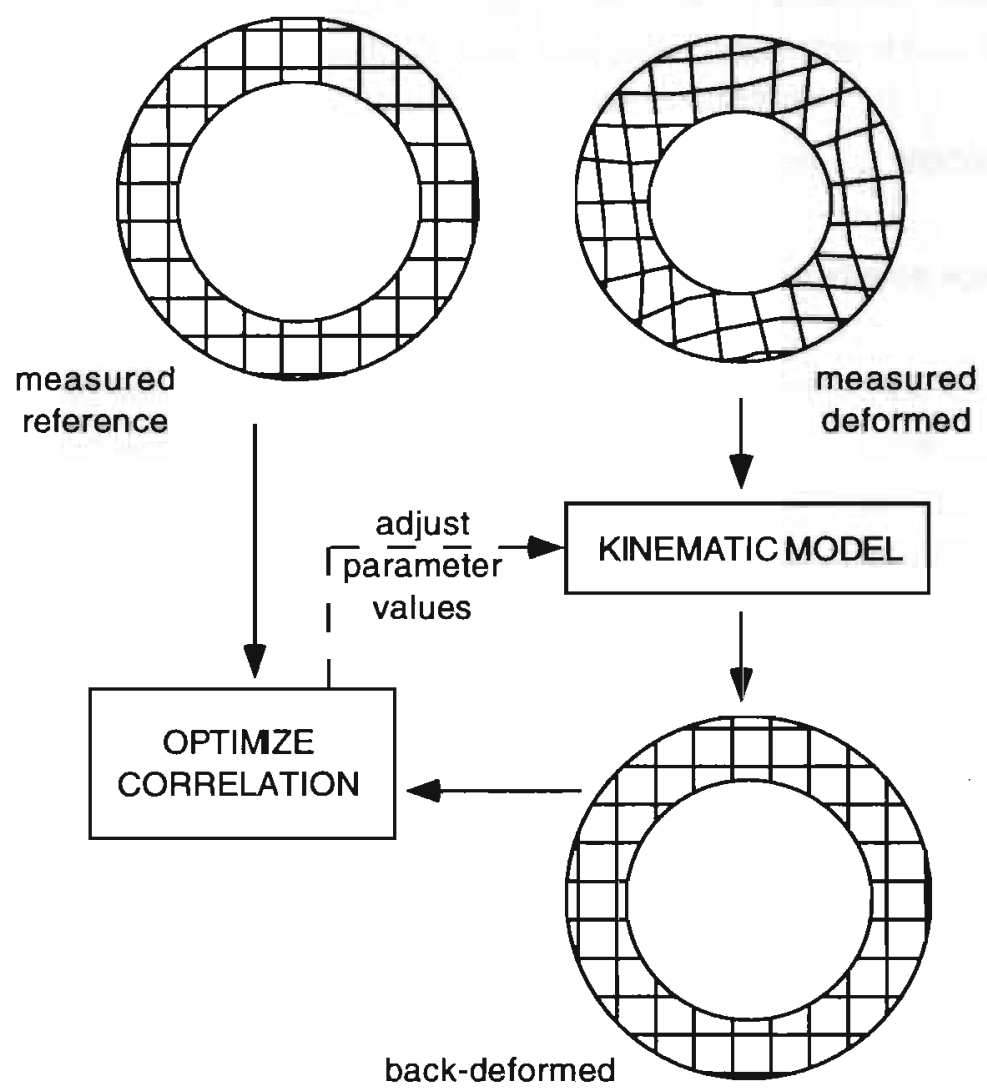

Figure 5.2 In the method proposed the deformed tagging pattern is deformed back to its initial, undeformed state using a kinematic model. Back-deformation is optimized for images of all cross-sections simultaneously, thus merging $2 D$ image information directly into $3 D$ deformation information. No separate tags need to be detected. Tags are only needed to provide sufficient contrast within the images. User-interaction is limited to delineation of left ventricular contours in images at the reference time.

first part of systole. The positions of 16 points, distributed over the mid-wall geometry of the left ventricle, formed the set of kinematic parameters, varying as a function of time. Motion elsewhere in the wall was obtained by an interpolation algorithm, based on the applied kinematic model. By simultaneous fit of all back-deformed images to the reference images, the positions of these 16 points were reconstructed as a function of time. Using the kinematic model, circumferential, longitudinal, and area 
strains were calculated in 12 different regions of the left ventricular wall. The method was evaluated in six healthy volunteers and two patients with myocardial infarction.

\subsection{METHODS}

\subsubsection{Image acquisition and preprocessing}

Magnetic Resonance Imaging was performed using a 0.5 Tesla MR imaging system (Philips Gyroscan T5-II, Philips Medical Systems, Best, The Netherlands) with body wrap-around coil. Six healthy volunteers and two patients having a myocardial infarction were imaged. One patient had an anterior wall infarction (patient $A$ ), and the other one had a posterior wall infarction (patient $P$ ). MR tagged images were acquired from two perpendicular long-axis cross-sections and two parallel short-axis crosssections, located about 2 and $4 \mathrm{~cm}$ below the left ventricular base at end-diastole. The ECG was used to trigger image acquisition. In each cross-section, prior to imaging a rectangular tagging grid with an inter tag distance of $5 \mathrm{~mm}$ was applied to the tissue by Spatial Modulation of the Magnetization (SPAMM), using two $90^{\circ}$ radiofrequency (RF) pulses for both orthogonal directions. Images were acquired at time intervals of $20 \mathrm{~ms}$, starting $20 \mathrm{~ms}$ after the R-wave in the ECG. Gradient-echo imaging (TR determined by cardiac frequency, TE $10 \mathrm{~ms}$, flip angle $40^{\circ}$ ) was utilized to acquire the images. The field of view was $213 \times 213 \mathrm{~mm}$ with a resolution of $256 \times 256$ pixels. Slice thickness was $8 \mathrm{~mm}$. Non-tagged images were acquired at the same cross-sections as the tagged images. Each time series of images of a crosssection was acquired in about 5 to 6 minutes.

T1 relaxation and tissue motion caused the tags in the MR tagged images to fade away slowly, until after about $200 \mathrm{~ms}$ noise became predominant. To reduce fading the tagging pattern was enhanced by spatial bandpass filtering of the images, as described in chapter 4 .

\subsubsection{Description of 3D left ventricular reference geometry}

Before quantifying the deformation of the left ventricular wall, first the geometry of the wall at a reference time was described (figure 5.3: $\mathrm{C}$ ). The time at which the first 


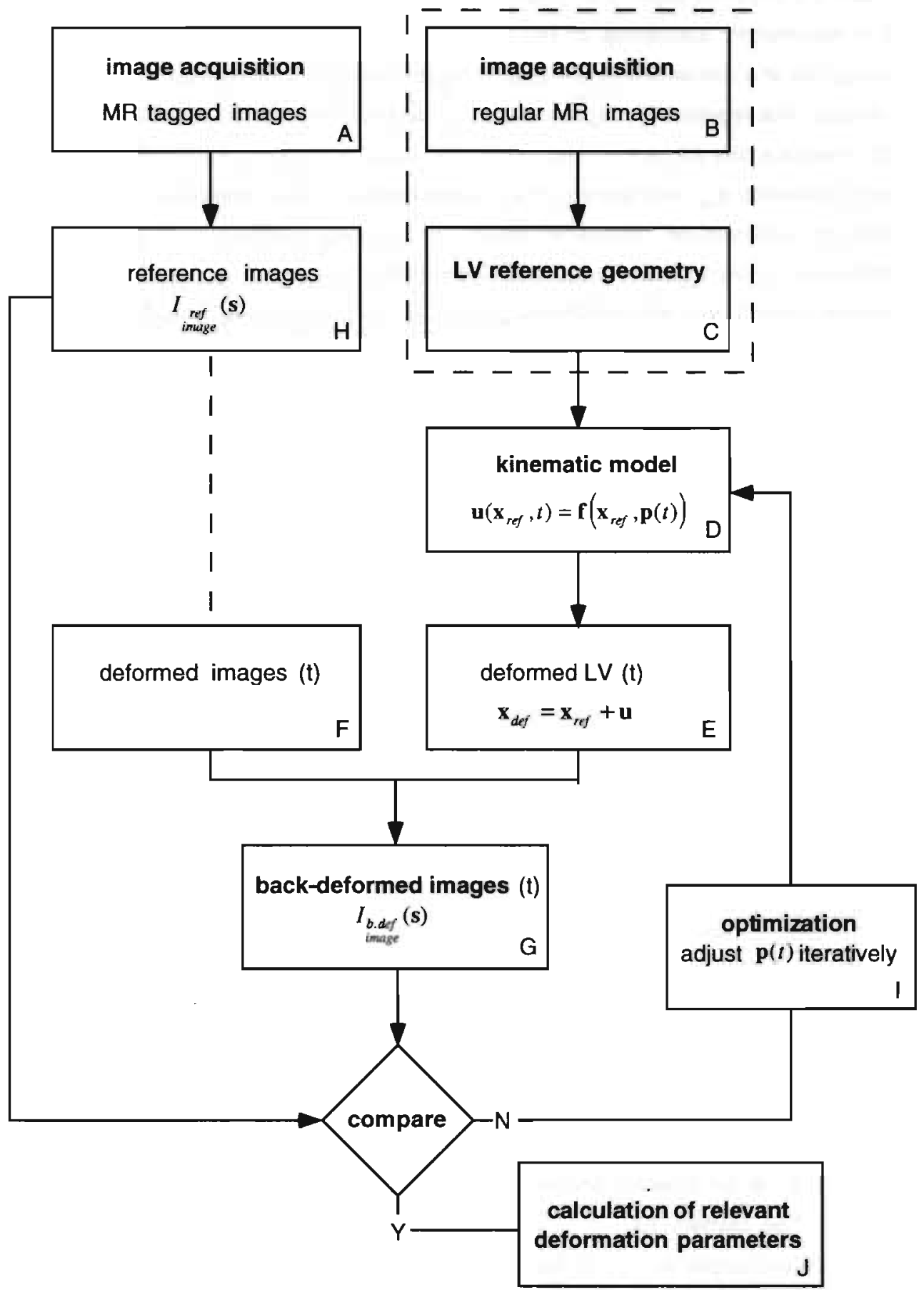


Figure 5.3 (previous page) Flow chart of the method proposed to determine left ventricular wall deformation. Sequences of MR cardiac tagged images (A), as well as regular MR images $(B)$ at a reference time, are acquired from a number of cross-sections through the left ventricle. The regular MR images are used to determine the $3 D$ left ventricular geometry at the reference time (C). A kinematic model $(D)$ is used to describe the displacement $\mathbf{u}$ of a point at position $\mathbf{x}_{r f}$ as a function of $\mathbf{x}_{r e f}$ and a number of parameters denoted by $\mathbf{p}$. In this way the deformed left ventricle at time $t$ is described mathematically $(E)$. The motion information is related to the image information on the cross-sections in order to deform the images at time $t$ (F) back to a reference state (G). The back-deformed images are compared with the acquired images at the reference time $(H)$, with respect to image gray values $I(\mathbf{s})$ at positions s. In an optimization procedure (I) the parameters $\mathbf{p}$ are adjusted iteratively in order to obtain the best match between back-deformed and reference images. Subsequently, the optimized kinematic parameters $\mathbf{p}$ are used to quantify relevant deformation parameters (J).

images at the four cross-sections were acquired, about $20 \mathrm{~ms}$ after the R-wave in the ECG, was chosen as reference time.

In order to describe the wall geometry mathematically, a cardiac coordinate system was defined as follows. The middle of the line connecting the centers of both shortaxis image slices was the origin. The $x$-and $y$-axes were chosen in the horizontal and the vertical direction of the short-axis images, respectively. Anatomically, the negative $x$-axis pointed towards the septal wall. The $z$-axis was chosen perpendicular to the $x$ and $y$-axes and pointed towards the base. Then, knowing the 3D locations of the image slices, the 3D location $x_{i}=\left(x_{i}, y_{i}, z_{i}\right)$ of pixel $i$ at position $s_{i}=\left(s_{1}, s_{2}\right)$ in image cross-section $j$ is known, with respect to the cardiac coordinate system:

$$
\mathbf{x}_{i}=\mathbf{x}_{i}\left(\mathbf{s}_{i}, j\right)
$$

The reference geometry of the left ventricle was represented by the mid-wall surface (figure 5.4). In an arbitrary short-axis cross-section the mid-wall was assumed to be circular. The centers $\left(X_{\text {ref }}, Y_{\text {ref }}\right)$ and the radii $R_{\text {ref }}$ of these circles depend on the longitudinal coordinate $(z)$ with fourth order polynomials: 


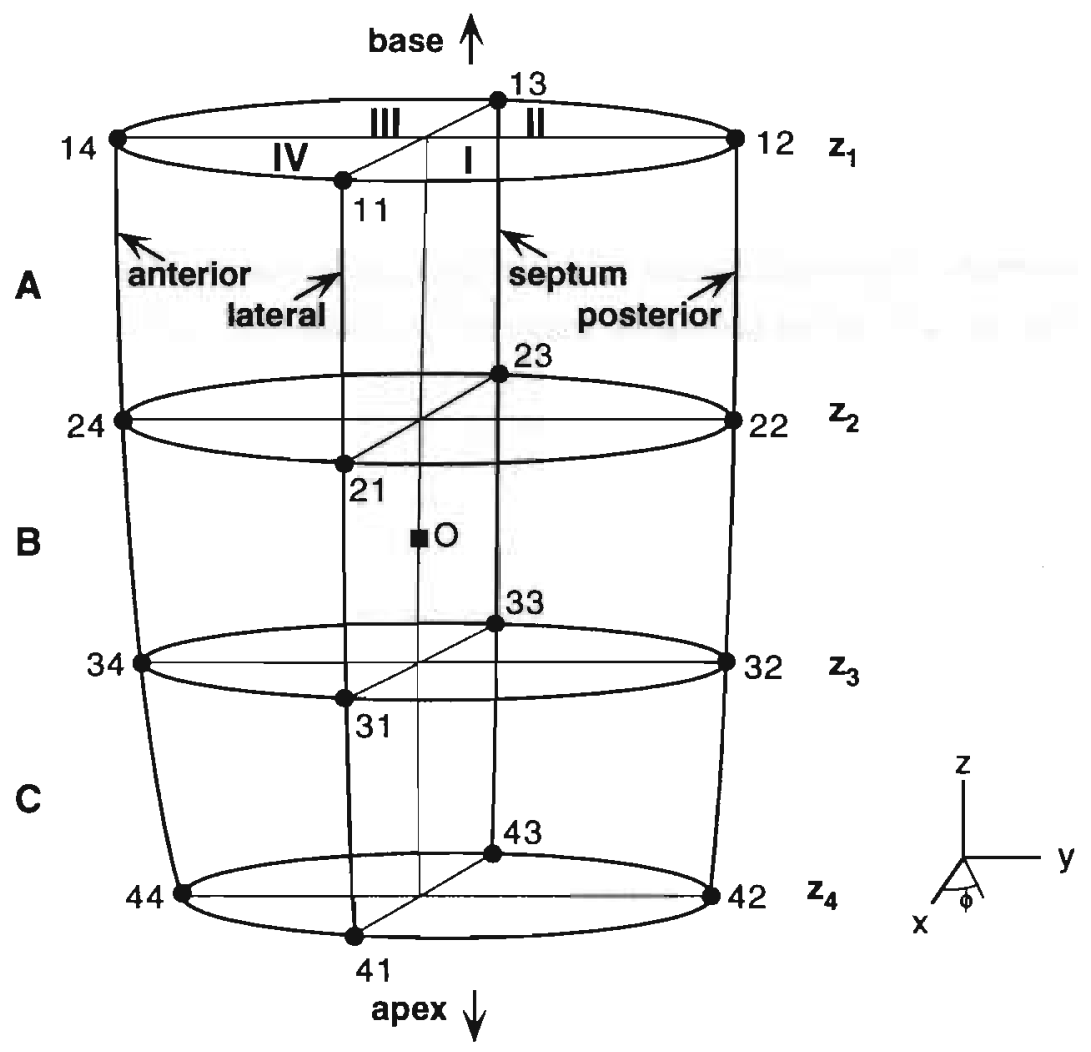

Figure 5.4 Schematic representation of the left ventricular mid-wall geometry at the enddiastolic reference time and indication of the 16 points that are used in the $3 D$ kinematic model to deduce local wall motion from. The left ventricle is divided into 4 quadrants $(1, I I, I I$, and $I V)$ along the circumference and 3 longitudinal sections ( $A, B$, and $C$ ), resulting in 12 different regions. Note that images are acquired from 2 short-axis $\left(z=z_{2}\right.$ and $\left.z=z_{3}\right)$ and two long-axis cross-sections. The point $O$ represents the origin of the coordinate system.

$$
\begin{aligned}
& X_{r e f}=X_{r e f}(z), \\
& Y_{r e j}=Y_{r e f}(z), \\
& R_{r e f}=R_{r e f}(z) .
\end{aligned}
$$

These polynomials were the result of a fit such that mid-wall geometry was closest to all pixels in the separate images that were part of the wall, excluding the apex 
(Appendix A). To this purpose, in all regular MR images at the reference time inner and outer wall contours were delineated manually.

\subsubsection{Kinematic model}

A 3D kinematic model was used to describe the displacement $\mathbf{u}=\left(u_{x}, u_{y}, u_{z}\right)$ of the left ventricular wall during systole as a function of position $\mathbf{x}_{r e f}=\left(x_{r e f}, y_{r e f}, z_{r e f}\right)$ and time (figure 5.3: D). For the current position $\mathrm{x}_{t}=\left(x_{t}, y_{t}, z_{t}\right)$ at time $t$ it holds:

$$
\mathbf{x}_{\iota}=\mathbf{x}_{\text {ref }}+\mathbf{u}\left(\mathbf{x}_{\text {ref }}, \mathbf{p}(t)\right)
$$

where $\mathbf{p}(t)$ represents a limited set of kinematic parameters, varying as a function of time.

We designed a kinematic model in which the displacement of an arbitrary point in the wall was derived from the motion of 16 points, evenly distributed throughout the wall. At the reference time the 16 points were located on the mid-wall surface (figure 5.4) at the positions $\mathbf{x}_{h q}=\left(x_{h p}, y_{h p}, z_{h}\right)$. It holds:

$$
\left(\begin{array}{c}
x_{h p} \\
y_{h p} \\
z_{h}
\end{array}\right)=\left(\begin{array}{c}
X_{r e f}\left(z_{h}\right) \\
Y_{r e f}\left(z_{h}\right) \\
z_{h}
\end{array}\right)+R_{r e f}\left(z_{h}\right)\left(\begin{array}{c}
\cos \left(\phi_{p}\right) \\
\sin \left(\phi_{p}\right) \\
0
\end{array}\right) ; \quad p=1,2,3,4 ; h=1,2,3,4,
$$

where $z_{1}=+20 \mathrm{~mm}, z_{2}=+10 \mathrm{~mm}, z_{3}=-10 \mathrm{~mm}$, and $z_{4}=-20 \mathrm{~mm}$ while $\phi_{1}=0, \phi_{2}=\pi / 2$, $\phi_{3}=\pi$, and $\phi_{4}=-\pi / 2$. The points are indexed $h p$, with $h$ and $p$ indicating the longitudinal and circumferential positions, respectively (figure 5.4). The displacements $\mathbf{u}\left(\mathbf{x}_{h_{p}}\right)$ of the 16 points were determined directly from the kinematic parameters, while subsequently the displacement $\mathbf{u}\left(\mathbf{x}_{r e f}\right)$ of an arbitrary point $\mathbf{x}_{r e f}$ in the wall was calculated by interpolation between the 16 points, as explained below. Displacements are expressed either in Cartesian $\left(\mathbf{u}=\left(u_{1}, u_{y}, u_{z}\right)\right)$ or in polar $\left(\mathbf{u}=\left(u_{r}, u_{\phi}, u_{z}\right)\right)$ coordinates ( $r$ : radial, $\phi$ : circumferential, $z$ : longitudinal), with $\left(X_{\text {ref }}(z), Y_{\text {ref }}(z)\right)$ as polar origin.

Parameters in the kinematic model were defined as follows. Displacements of the 8 points in the planes $z=z_{2}$ and $z=z_{3}$ along the Cartesian axes $(x, y, z)$ constituted 24 
parameters. No information was available about circumferential displacement in the planes $z=z_{3}$ and $z=z_{4}$. At these short-axis cross-sections in-plane motion of the centroids $\left(\Delta X\left(z_{h}\right), \Delta Y\left(z_{i n}\right)\right)$ of the mid-wall circles and the changes of the radii $\Delta R\left(z_{h}\right)$ constituted 6 parameters describing radial displacements of the points $\mathrm{x}_{h p}$. Another parameter in the kinematic model was the radial gradient in radial displacement $g_{r}$, needed to describe motion of points outside the mid-wall surface. In total, we thus obtained 31 parameters in the kinematic model, ordered in a vector $\mathbf{p}$ :

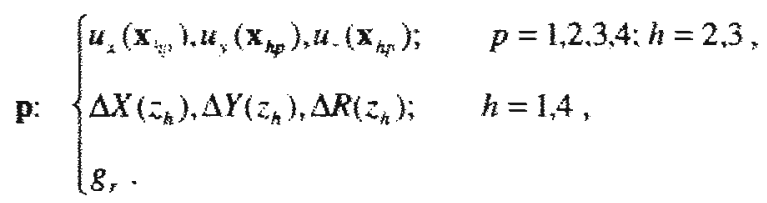

From the kinematic parameters $\mathbf{p}$ we calculated 3D displacements $\mathbf{u}\left(\mathbf{x}_{h p}\right)$ of all 16 points $\mathbf{x}_{h p}$ :

$$
\mathbf{u}\left(\mathbf{x}_{h p}\right)=f\left(\mathbf{x}_{h p}, \mathbf{p}\right) ; \quad p=1,2,3,4 ; h=1,2,3,4
$$

This was done as follows. The displacements $\left(u_{x}\left(\mathbf{x}_{h p}\right), u_{y}\left(\mathbf{x}_{h p}\right)\right)$ in the planes $z=z_{2}$ and $z=z_{3}$ were first converted to polar coordinates $\left(u_{r}\left(\mathbf{x}_{h^{\prime}}\right), u_{\varphi}\left(\mathbf{x}_{h^{\prime}}\right)\right)$, taking into account the displacements $\left(\Delta X\left(z_{h}\right), \Delta Y\left(z_{h}\right)\right)$ of the polar origin at each cross-section, which were calculated by fitting circles through the displaced points. Circumferential displacements of the points with $z=z_{1}$ and $z=z_{4}$ were assumed to be equal to those of the corresponding (equal $p$ ) points with $z=z_{2}$ and $z=z_{3}$, respectively. Longitudinal displacements of the points $x_{h p}$ in the planes $z=z_{1}$ and $z=z_{4}$ were calculated assuming constant longitudinal strain in the longitudinal direction. The detailed derivation of equation (6) is given in Appendix B.

Subsequently, displacement of an arbitrary point $\mathbf{x}_{r \text { re }}$ in the wall to $\mathbf{x}_{r}$ was determined from the displacements of the 16 points $x_{h p}$ by means of interpolation. To this purpose, radial $u_{r}\left(\mathbf{x}_{r f f}\right)$, circumferential $u_{\phi}\left(\mathbf{x}_{r e f}\right)$, and longitudinal $u_{\xi}\left(\mathbf{x}_{r e f}\right)$ displacements of $\mathbf{x}_{r e f}$, as well as displacement of the polar origin $\left(\Delta X\left(z_{\text {sef }}\right), \Delta Y\left(z_{\text {ref }}\right)\right)$ at $z_{\text {ref }}$, were calculated as a function of $\mathbf{x}_{r e f}$ and the parameters $\mathbf{p}$ (Appendix $\mathrm{C}$ ). This results in the following expression for the coordinates of $\mathbf{x}_{1}$ : 


$$
\mathbf{x}_{t}=\left(\begin{array}{c}
X_{r e f}\left(z_{r e f}\right)+\Delta X\left(z_{r e f}\right)+\left(R_{r e f}\left(z_{r e f}\right)+\Delta r_{m w}+u_{r}\left(\mathbf{x}_{r e f}\right)\right) \cos \left(\phi_{r e f}+u_{\phi}\left(\mathbf{x}_{r e f}\right)\right) \\
Y_{r e f}\left(z_{r e f}\right)+\Delta Y\left(z_{r e f}\right)+\left(R_{r e f}\left(z_{r e f}\right)+\Delta r_{n w}+u_{r}\left(\mathbf{x}_{r e f}\right)\right) \sin \left(\phi_{r e f}+u_{\phi}\left(\mathbf{x}_{r e f}\right)\right) \\
z_{r e f}+u_{z}\left(\mathbf{x}_{r e f}\right)
\end{array}\right),
$$

with $\Delta r_{m w}$ the radial distance of the point $\mathbf{x}_{r e}$ to the reference mid-wall surface.

\subsubsection{Back-deformation of MR-tagged images}

As described in chapter 4, images were deformed back to a reference state (figure 5.3: G) using the kinematic model. Briefly recapitulating, first the relation between image information and motion information was described. Consider a pattern of tags in the reference state in one of the cross-sections. In 3D space a tag extends with about equal intensity along a line perpendicular to the reference image plane. Due to cardiac motion the tag deforms and displaces in 3D space, but is detected only in the fixed 2D image plane. Consequently a point $A_{r e f}$ in the image plane in the reference state moves out of this plane to $A_{t}$. By back-deformation, there is an observed point $B_{1}$ in the image plane at time $t$ that came from a position out of the image plane in the reference state $\left(B_{r e f}\right)$, but was on the same tag line perpendicular to the image plane as $A_{\text {ref }}$. Ideally, if no fading occurred, $B_{i}$ has the same image intensity as point $A_{\text {ref }}$. This knowledge was used in developing the algorithm to describe the backdeformation of the MR tagged images to the reference state (par. 4.4.2).

\subsubsection{Optimization of kinematic parameter values}

In order to determine 3D wall motion from the MR-tagged images, the back-deformed (b.def ) images of the four cross-sections were simultaneously compared with their respective reference ( $r e f)$ images by evaluating the following objective function $(F)$, for each time $t$ :

$$
F(\mathbf{p}(t))=\sqrt{\frac{1}{N} \sum_{j=1}^{4} \sum_{i=1}^{N}\left|I_{\text {b.def }}^{N}\left(\mathbf{p}(t), \mathbf{s}_{i}, j\right)-I_{\substack{\text { ref } \\ \text { image }}}\left(\mathbf{s}_{i}, j\right)\right|^{2}},
$$

with $\mathrm{s}_{i}$ the position of pixel $i$ in cross-section $j, N_{j}$ the total number of pixels in cross-section $j$ within the wall region between inner and outer contours, 
$N=N_{1}+N_{2}+N_{3}+N_{4}, I$ the pixel gray value, and $\mathbf{p}$ the vector of 31 kinematic parameters. The objective function was minimized by variation of the kinematic parameter values (figure 5.3: I) following Powell's method (Press et al., 1988). As a result a best fit was found between the back-deformed images and the reference images.

\subsubsection{Calculation of deformation of the left ventricular wall}

From the displacements of the 16 points $\mathbf{x}_{h p}$ circumferential $\left(\varepsilon_{\phi}\right)$, longitudinal $\left(\varepsilon_{z}\right)$, and surface area $\left(\varepsilon_{\text {ureal }}\right)$ strains were calculated (Appendix D; figure 5.3: J). This was done for 12 different regions defined as follows. The reference angles $\phi_{p}$ of the 16 points, which were equal to $-\pi / 2,0, \pi / 2$, or $\pi$, divided the left ventricle into four quadrants along the circumference (figure 5.4: I, II, III, and IV). Furthermore, the 16 points were located on four different reference planes with constant $z=z_{k}$, dividing the left ventricle into three longitudinal sections (figure 5.4: $A, B$, and $C$ ).

Torsion of the left ventricle was calculated as the difference in average rotation between the lower $\left(z=z_{3}\right)$ and the upper $\left(z=z_{2}\right)$ short-axis cross-sections, each multiplied by their respective radii, and divided by the longitudinal distance between the cross-sections, all at times $t$. Physically, this measure of torsion may be interpreted as the shear angle on the mid-wall surface between both cross-sections.

To describe the effect of asymmetry in the deformation pattern, differences in area strain values along the circumference were assessed. This was done by determining the first circular harmonic (i.e. a cosine function with amplitude $\Delta \mathrm{E}$ and maximum at $\left.\phi_{\max }\right)$ through the area strain values of the four regions along the circumference, with respect to the angle $(\phi)$. The amplitude of this cosine function was normalized to the absolute value of the average area strain value $\left(\mathrm{E}_{\text {average }}\right)$ of that particular longitudinal section. Thus, for the normalized cosine function $\left(\bar{E}_{\text {area }}\right)$ it holds:

$$
\overline{\mathrm{E}}_{\text {area }}(\phi)=\frac{\Delta \mathrm{E}}{\left|\mathrm{E}_{\text {arerage }}\right|} \cdot \cos \left(\phi-\phi_{\text {max }}\right) .
$$




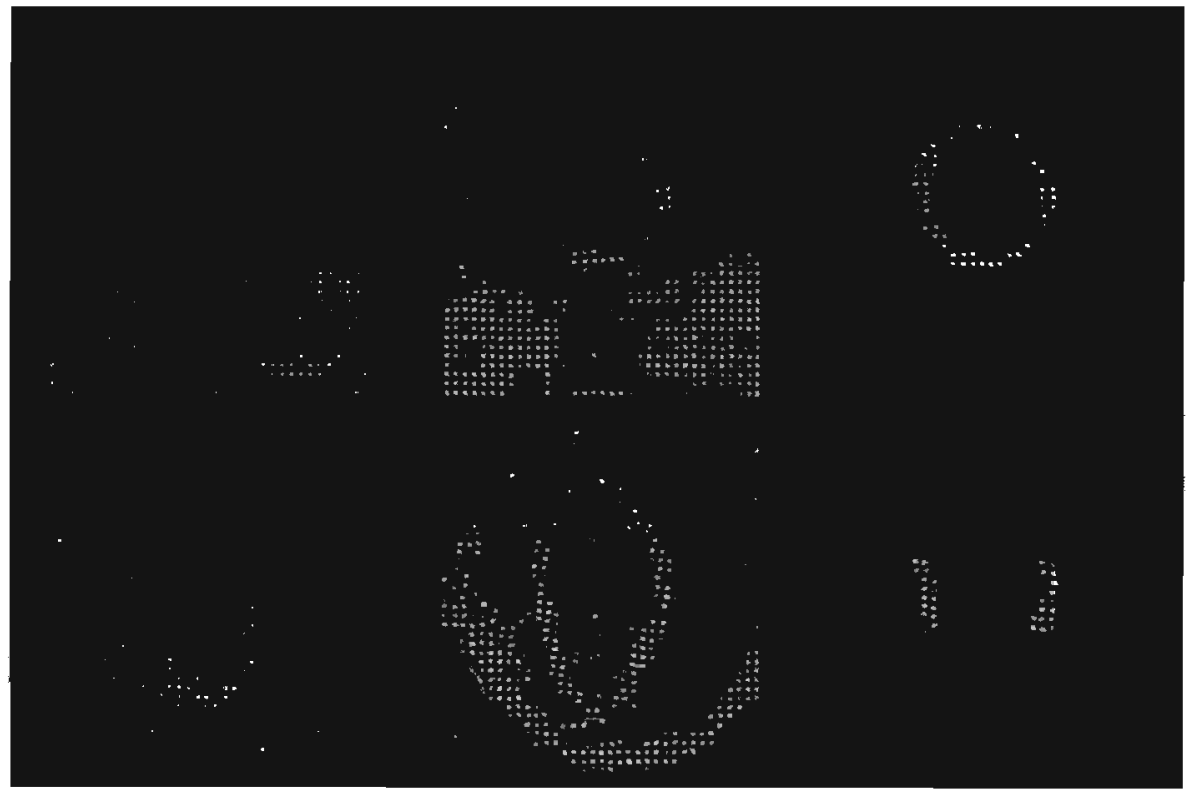

Figure 5.5 Representative original (left), bandpass filtered (middle), and back-deformed (right) short-axis (above; as viewed from apex) and long-axis (below) images, $140 \mathrm{~ms}$ after the $R$-wave in the ECG. Compare with figure 5.2 .

\subsection{RESULTS}

In figure 5.5 the original, bandpass filtered, and back-deformed images are shown. Filtering enhanced the tagging pattern especially for the images acquired relatively late after application of the tags. The back-deformed images resembled the initial rectangular tagging grid.

Analysis was performed at 8 moments in time from 20 to $160 \mathrm{~ms}$ after the R-wave in the ECG. Circumferential, longitudinal, and area strains from the beginning to the end of the time interval were averaged over the 12 regions of the left ventricular wall (figure 5.4). Average mid-wall circumferential strains were $-0.105 \pm 0.025$ (mean $\pm s d$ ) for the 6 volunteers and -0.076 and -0.064 for patient $A$ (anterior wall infarction) and patient $P$ (posterior wall infarction), respectively. Longitudinal strains averaged $-0.079 \pm 0.018$ over the 12 regions for the volunteers and -0.071 and -0.046 for patient $A$ and patient $P$, respectively. Area strains averaged $-0.176 \pm 0.039$ for the volunteers, 


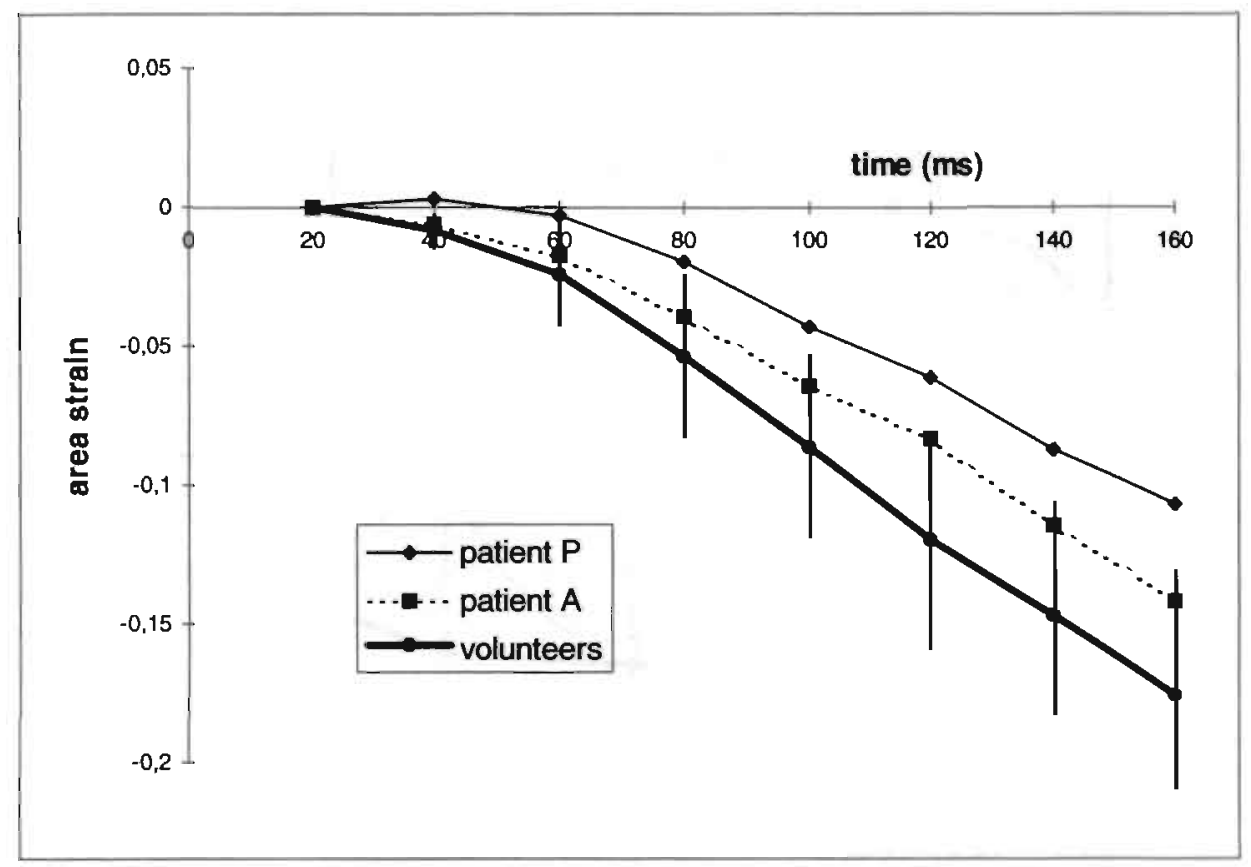

Figure 5.6 Time courses of area strains, averaged over the 12 regions of the left ventricular wall (figure 5.4), for a patient with anterior wall infarction (A), a patient with posterior wall infarction $(P)$, and the average values of the six volunteers. Time zero refers to the $R$-wave in the ECG. The error bars indicate the standard deviation of the values of the six volunteers.

-0.142 for patient $A$, and -0.107 for patient $P$. Time courses of the latter strains are shown in figure 5.6 for the volunteers and the patients. Torsion was $0.068 \pm 0.043 \mathrm{rad}$ for the volunteers and $0.066 \mathrm{rad}$ and $0.053 \mathrm{rad}$ for patient $\mathrm{A}$ and patient $\mathrm{P}$, respectively. Radial displacements, averaged over the 12 regions, were $-2.98 \pm 0.67$ $\mathrm{mm}$ for the volunteers, $-2.10 \mathrm{~mm}$ for patient $A$, and $-2.09 \mathrm{~mm}$ for patient $P$. Average wall thickening, calculated as the radial gradient in radial displacement $\left(g_{r}\right)$, was $0.085 \pm 0.027$ for the volunteers. For the two patients these gradients were near zero: -0.004 for patient $A$ and 0.007 for patient $P$. Longitudinal displacement of the whole left ventricle was $-4.34 \pm 0.89 \mathrm{~mm},-3.05 \mathrm{~mm}$, and $-5.44 \mathrm{~mm}$, for the volunteers, patient $A$ and patient $P$, respectively. 

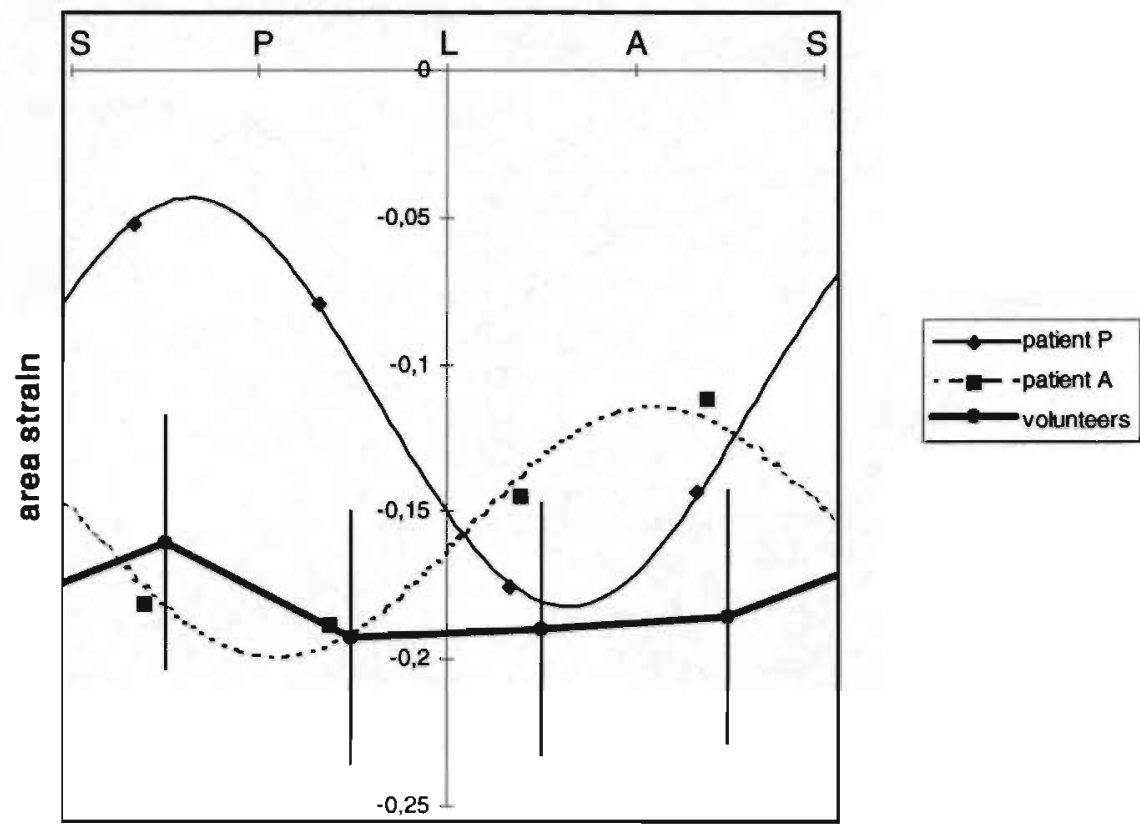

Figure 5.7 Area strain values along the circumference (S: septal wall, A: anterior wall, L: lateral wall, $P$ : posterior wall) at long-axis section $B$ for a patient with anterior wall infarction $(A)$, a patient with posterior wall infarction $(P)$, and the average values of the six volunteers, $160 \mathrm{~ms}$ after the R-wave in the ECG. The error bars indicate the standard deviation of the values of the six volunteers. Cosines are fitted through the values of the two patients. Note that for the patients the area strains are maximum (=minimum contraction) in their respective infarcted regions.

In figure 5.7 differences in area strain values along the circumference at longitudinal section B, at $160 \mathrm{~ms}$ after the R-wave in the ECG, are shown for the volunteers and for the patients. For the volunteers the amplitudes of the normalized cosines $\left(\overline{\mathrm{E}}_{\text {arra }}\right.$; Equation 9) fitted to the area strain values along the circumference were $11.7 \pm 12.7 \%$, $9.1 \pm 5.1 \%$, and $18.3 \pm 8.5 \%$ for the longitudinal sections $A, B$, and $C$, respectively. For patient $A$ these normalized cosine amplitudes were $23.6 \%, 27.2 \%$, and $43.6 \%$, and for patient $P 60.4 \%, 61.5 \%$, and $80.1 \%$, respectively. The values for all six volunteers and the two patients are illustrated in figure 5.8. The amplitude and angular location of the maximum of the normalized cosine are represented by the radial and angular 

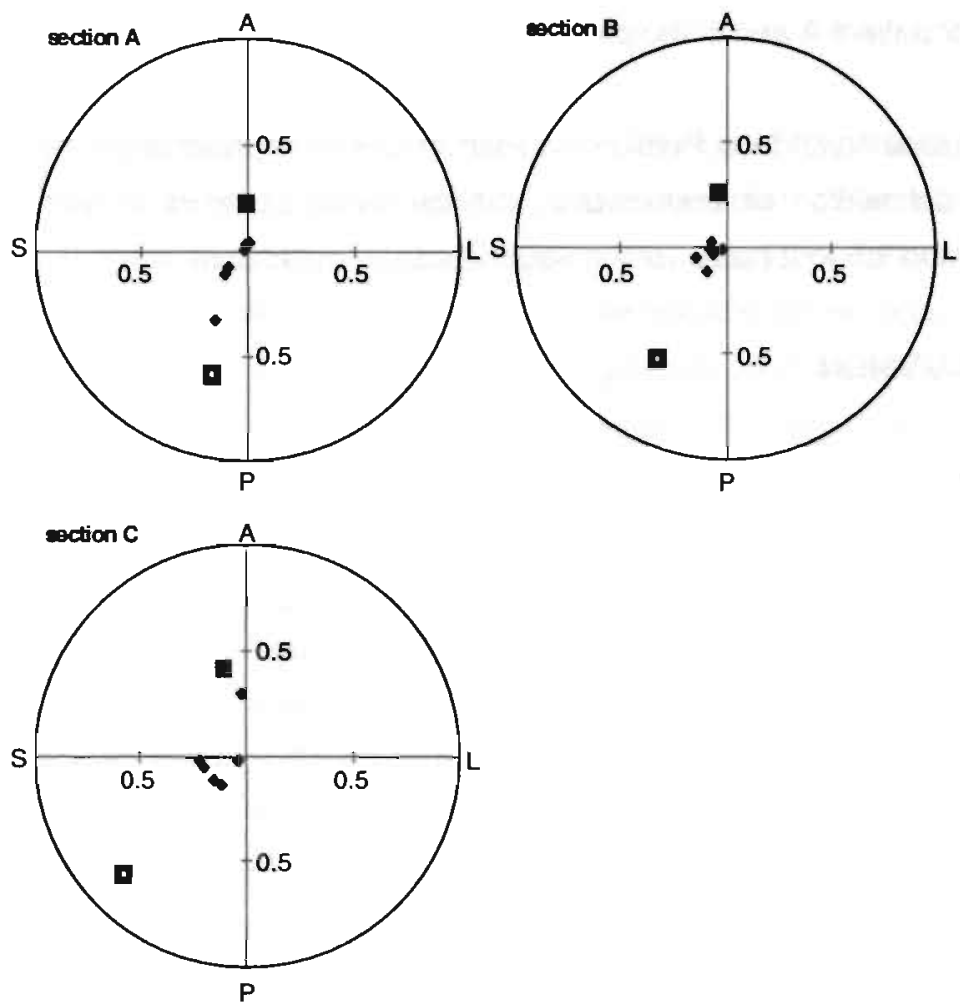

Figure 5.8 Location of lowest contractile function, i.e. least negative area strain, along the circumference as viewed from the apex, at $160 \mathrm{~ms}$ after the R-wave in the ECG. The distance of a point to the origin indicates the amplitude of a cosine fitted to the area strain values along the circumference, and normalized to the average value along the circumference. The angle between the point and the horizontal axis indicates the location of the region with lowest contractile function along the circumference (S: septal wall, A: anterior wall, $L$ : lateral wall, $P$ : posterior wall). The sections $A, B$, and $C$ are defined in figure 5.4 . Section $B$ is the longitudinal section at which strains can be determined most accurately. The symbol $\mathbf{\square}$ indicates the patient with posterior wall infarction, $\square$ indicates the patient with anterior wall infarction, and the six $\bullet$ indicate the healthy volunteers.

coordinate relative to the origin, respectively. The maximum value of the cosine indicates least negative area strain, or region with lowest contractile function. For the volunteers generally a slight maximum was found in the septal region (figure 5.8). For 
the patients this maximum was more pronounced, and was located in the anterior region for patient $A$ and in the posterior region for patient $P$.

Analysis time on an Intel Pentium $133 \mathrm{MHz}$ PC was approximately 10 minutes for the manual delineation of endocardial and epicardial contours in the four reference images, and 1.5 to 2 hours for the automated kinematic analysis.

\subsection{DISCUSSION}

A method is presented to quantify left ventricular wall deformation by means of MR cardiac tagging. Using a 3D kinematic model images are deformed back to a reference state in order to estimate deformation. Images from multiple cross-sections are analyzed simultaneously, thus merging 2D image information directly into 3D deformation information. No individual tags need to be detected. The only manual interference is the delineation of left ventricular wall contours at a single reference time. Furthermore, the method may also be used for analysis of MR-tagged images with different tag shapes. The feasibility of this method was shown by the fact that deformation values as obtained in volunteers were in the range of previously reported values, considering the fact that our measurements did not cover the entire systolic phase (Buchalter et al., 1990; Clark et al., 1991; Aelen et al., 1997; Rademakers and Bogaert, 1997). Moreover, in patients the regions with minimum deformation coincided with the location of the myocardial infarction.

In a comparative study of myocardial strains by Azhari et al. (Azhari et al., 1995) area strain was found to be the best discriminating index between healthy and dysfunctional tissue. Therefore, we used area strain to locate the region with lowest contractile function. Normalization to the average was used to increase sensitivity in detecting the dysfunction in patients. This is because in case of a dysfunction normal contraction is generally less, while the aberrant component of motion increases. The most pronounced differences in non-uniformity of area strains between volunteers and patients were found near the equator at longitudinal section $B$ (figure $5.4 ; \overline{\mathrm{E}}_{\text {area }}$ (equation 9): volunteers $9.1 \pm 5.1 \%$, versus $27.2 \%$ and $61.5 \%$ for patient $A$ and patient $P$, respectively). In this section strains could be determined most accurately. The fact that the location of lowest contractile function found by us matched with the 
circumferential location of the infarction in 2 patients shows that our method can be used correctly to detect and localize a motional dysfunction.

Most other methods to quantify left ventricular wall deformation by means of MR cardiac tagging rely on detection of individual tags (Guttman et al., 1994; Kumar and Goldgof, 1994; Kraitchman et al., 1995). Following this approach, left ventricular epicardial and endocardial wall contours have to be delineated in all separate images. Despite the large amount of effort that has been expended on the problem, at the present state of the art it is not possible to delineate the wall contours fully automatically, and manual corrections are still needed (Guttman et al., 1994; Axel et al., 1995; McVeigh, 1998). Using our method, which does not require detection of individual tags, wall contours need only be detected at a single reference time. Another common problem in tag detection is that the applied method has to be finetailored to the specific images (tag shape, image quality/contrast) used, in order to ensure accurate tracking of the tags (Guttman et al., 1994; Kumar and Goldgof, 1994).

Prince and McVeigh (Prince and McVeigh, 1992) developed a variable brightness optical flow (VBOF) method to track the motion within the myocardium from tagged MR images. As with the method we propose, no separate tags needed to be detected. A limitation of the VBOF method is that errors in motion estimates accumulate when sequential images are tracked. In addition, when using the VBOF method, a number of image properties, such as the variable brightness and noise in the images, must be known a priori. Our method does not need this a priori knowledge. Back-deformed images were fitted to reference images in which the tagging pattern is very regular. Even when the overall brightness in the images diminished a well defined best fit could be found.

The present study was meant to show the applicability of the proposed approach to study inhomogeneous cardiac wall deformation. As a first step, images were acquired from only two short-axis and two long-axis cross-sections. A possible major extension of the method in the near future is to acquire images from more cross-sections (McVeigh and Atalar, 1992; O'Dell et al., 1995). Such an improvement in spatial resolution of the imaging, in combination with a more detailed kinematic model and description of reference geometry (Creswell et al., 1994), would render more detailed 
information about regions of dysfunction. In addition, more advanced tagging and imaging techniques may be used, such as CSPAMM (Fischer et al., 1993), breathhold cine MRI (McVeigh and Atalar, 1992) or echo-planar imaging (Tang et al., 1995), with which tagging contrast can be enhanced considerably. The method we propose can also be employed for analyzing cardiac wall deformation using any of these techniques. For instance, there is no principle limitation to apply our method to line tagged images instead of images with a tagging grid. Tagging was only used to provide sufficient contrast in the images.

In the present study no particular effort was made to reduce the time needed to analyze the images. For instance, we used Powell's method to determine the optimal fit between back-deformed and reference images. Although this method is quite robust, we think that methods more dedicated to our specific application, in combination with more powerful computers, will decrease analysis time considerably.

In conclusion, the feasibility was shown of a new approach to quantify inhomogeneous cardiac wall motion by means of MR cardiac tagging. The method determined $3 D$ wall deformation directly from the images, without the need to detect individual tags. Images from multiple cross-sections were analyzed simultaneously, thus merging $2 \mathrm{D}$ image information directly into $3 \mathrm{D}$ deformation information. The method is highly automated, only requiring manual delineation of left ventricular contours at a single reference time, and may be used to analyze MR-tagged images with different tag shapes.

\section{REFERENCES}

Aelen, F. W. L., Arts, T., Sanders, D. G. M., Thelissen, G. R. P., Muijtjens, A. M. M., Prinzen, F. W., and Reneman, R. S. (1997) Relation between torsion and crosssectional area change in the human left ventricle. J Biomechanics 30, 207-212.

Axel, L., Bloomgarden, D. C., Chang, C.-N., Fayad, Z. A., Kraitchman, D. L., and Young, A. A. (1995) An integrated program for 2-D and 3-D analysis of heart wall motion from Magnetic Resonance Imaging. In Computers in Cardiology. . Vienna: IEEE.

Axel, L. and Dougherty, L. (1989) MR Imaging of Motion with Spatial Modulation of the Magnetization. Radiology 171, 841-845.

Azhari, H., Oliker, S., Rogers, W. J., Weiss, J. L., and Shapiro, E. P. (1996) Three- 
dimensional mapping of acute ischemic regions using artificial neural networks and tagged MRI. IEEE Trans Biomed Eng 43, 619-626.

Azhari, H., Weiss, J. L., Rogers, W. J., Siu, C. O., and Shapiro, E. P. (1995) A noninvasive comparative study of myocardial strains in ischemic canine heart using tagged MRI in 3-D. Am J Physiol 268, H1918-H1926.

Buchalter, M. B., Weiss, J. L., Rogers, W. J., Zerhouni, E. A., Weisfeldt, M. L., Beyar, R., and Shapiro, E. P. (1990) Noninvasive Quantification of Left Ventricular Rotational Deformation in Normal Humans Using Magnetic Resonance Imaging Myocardial Tagging. Circulation 81, 1236-1244.

Clark, N. R., Reichek, N., Bergey, P., Hoffman, E. A., Brownson, D., Palmon, L., and Axel, L. (1991) Circumferential Myocardial Shortening in the Normal Human Left Ventricle - Assessment by Magnetic Resonance Imaging Using Spatial Modulation of Magnetization. Circulation 84, 67-74.

Creswell, L. L., Moulton, M. J., Wyers, S. G., Pirolo, J. S., Fishman, D. S., Perman, W. H., Myers, K. W., Actis, R. L., Vannier, M. W., Szabo, B. A., et al. (1994) An experimental method for evaluating constitutive models of myocardium in in vivo hearts. Am J Physiol 267, H853-H863.

Fischer, S. E., McKinnon, G. C., Maier, S. E., and Boesiger, P. (1993) Improved Myocardial Tagging Contrast. Magn Reson Med 30, 191-200.

Guttman, M. A., Prince, J. L., and McVeigh, E. R. (1994) Tag and contour detection in tagged MR images of the left ventricle. IEEE T Med Imaging 13, 74-88.
Kraitchman, D. L., Young, A. A., Chang, C.-N., and Axel, L. (1995) Semi-automatic tracking of myocardial motion in MR-tagged images. IEEE T Med Imaging 14, 422-433.

Kramer, C. M., Lima, J. A. C., Reichek, N., Ferrari, V. A., Llaneras, M. R., Palmon, L. C., Yeh, I.-T., Tallant, B., and Axel, L. (1993) Regional differences in function within noninfarct myocardium during left ventricular remodeling. Circulation $\mathbf{8 8}$, 1279-1288.

Kumar, S. and Goldgof, D. (1994) Automatic tracking of SPAMM grid and the estimation of deformation parameters from cardiac MR images. IEEE T Mod Imaging 13, 122132.

Lima, J. A. C., Ferrari, V. A., Reichek, N., Kramer, C. M., Palmon, L., Llaneras, M. R., Tallant, B., Young, A. A., and Axel, L. (1995) Segmental motion and deformation of transmurally infarcted myocardium in acute postinfarct period. Am J Physiol 268, H1304-H1312.

Marcus, J. T., Gotte, J. W., Van Rossum, A. C., Kuijer, J. P. A., Heethaar, R. M., Axel, L., and Visser, C. A. (1997) Myocardial function in infarcted and remote regions early after infarction in man: Assessment by Magnetic Resonance tagging and strain analysis. Mag Res Med 38, 803-810.

McVeigh, E. R. (1996) MRI of myocardial function: motion tracking techniques. Magn Reson Imaging 14, 137-150.

McVeigh, E. R. (1998) Regional myocardial function. Cardiac Magnetic Resonance 16, 189-205.

McVeigh, E. R. and Atalar, E. (1992) Cardiac Tagging with Breath-Hold Cine MRI. Magn Res Med 28, 318-327.

O'Dell, W. G., Moore, C. C., Hunter, W. C., Zerhouni, E. A., and McVeigh, E. R. (1995) 
Three-dimensional

myocardial deformations: calculation with displacement field fitting to tagged MR images. Radiology 195, 829-835.

Press, W. H., Flannery, B. P., Teukolsky, S. A., and Vetterling, W. T. (1988) Numerical recipes - The an of scientific computing. Cambridge University Press, Cambridge.

Prince, J. L. and McVeigh, E. R. (1992) Motion estimation from tagged MR image sequences. IEEE T Med Imag 11, 238-249.

Prinzen, F. W., Arts, T., Hoeks, A. P. G., and Reneman, R. S. (1989) Discrepancies between Myocardial Blood Flow and Fiber Shortening in the Ischemic Border Zone as assessed with Video Mapping of Epicardial Deformation. Eur J of Physiology 415, 220229.
Rademakers, F. E. and Bogaert, J. (1997) Left ventricular myocardial tagging. Int $J$ Cardiac Imaging 13, 233-245.

Tang, C., McVeigh, E. R., and Zerhouni, E. A. (1995) Multi-shot EPI for improvement of myocardial tagging contrast: comparison with segmented SPGR. Magn Res Med 33, 443-447.

Young, A. A., Kramer, C. M., Ferrari, V. A., Axel, L., and Reichek, N. (1994) Threedimensional left ventricular deformation in hypertrophic cardiomyopathy. Circulation 90, 854-867.

Zerhouni, E. A., Parish, D. M., Rogers, W. J., Yang, A., and Shapiro, E. A. (1988) Human Heart: Tagging with MR Imaging - A Method for Noninvasive Assessment of Myocardial Motion. Radiology 169, 59-63.

\section{Appendix A: reference mid-wall geometry}

In an arbitrary short-axis cross-section the mid-wall was assumed to be circular. The centers $\left(X_{r e f}, Y_{r e f}\right)$ and radii $\left(R_{r e f}\right)$ of the mid-wall circles were described by fourth order polynomial series in the longitudinal coordinate $\mathbf{z}$ :

$$
\left(\begin{array}{c}
X_{r f f}(z) \\
Y_{r f f}(z) \\
R_{r e f}(z)
\end{array}\right)=\sum_{m=0}^{4}\left(\begin{array}{l}
\alpha_{m} \\
\beta_{m} \\
\gamma_{m}
\end{array}\right)\left(\frac{z}{z_{\max }-z_{\min }}\right)^{m}
$$

with $\alpha_{m}, \beta_{m}$, and $\gamma_{m}$ the polynomial coefficients, and $z_{\text {nux }}=+30 \mathrm{~mm}$ and $z_{\text {nü }}=-30 \mathrm{~mm}$.

To fit the mid-wall to the images of the wall in each cross-section $j$ a region of interest $\left(R O I_{j}\right)$ was delineated, representing the wall region between inner and outer contours. In the long-axis cross-sections the basal $z>z_{\min }$ and apical $z<z_{\min }$ regions were excluded from $R O I_{j}$. The polynomial coefficients were estimated from the 
images by minimizing the following objective function using Powell's method (Press et al., 1988):

$$
\begin{aligned}
F_{\text {geom }}\left(\alpha_{j}, \beta_{j}, \gamma_{j}\right) & =\sqrt{\frac{1}{N} \sum_{j=1}^{4} \sum_{i=1}^{N_{j}}\left(r_{i}-R_{r e f}\left(z_{i}\right)\right)^{2}}, \text { with } \\
r_{i} & =\sqrt{\left(x_{i}-X_{r e f}\left(z_{i}\right)\right)^{2}+\left(y_{i}-Y_{r e j}\left(z_{i}\right)\right)^{2}},
\end{aligned}
$$

where $\left(x_{i}, y_{i}, z_{i}\right)$ indicates the 3D location of the $i^{\text {th }}$ pixel in $R O I_{j}, N_{j}$ is the number of pixels in $R O I_{j}$, and $N=N_{1}+N_{2}+N_{3}+N_{4}$.

\section{Appendix B: motion of 16 points distributed throughout the wall}

Displacements were expressed in polar coordinates around the respective centers of the mid-wall circles. Circumferential displacements of the points $\mathbf{x}_{h \varphi}$ in the planes $z=z_{1}$ and $z=z_{4}$ were assumed to be equal to those of the corresponding points (equal $p$ ) in the planes $z=z_{2}$ and $z=z_{3}$, respectively. First, $2 \mathrm{D}$ motion $\left(\Delta X\left(z_{h}\right), \Delta Y\left(z_{h}\right)\right)$ of the polar origins in the short-axis cross-sections with $z=z_{2}$ and $z=z_{3}$ was estimated by least squares fitting a circle to the four displaced points $\left(x_{h p}+u_{x}\left(\mathbf{x}_{h p}\right), y_{h p}+u_{y}\left(\mathbf{x}_{h p}\right)\right)$ in a cross-section. Displacement of the center of a circle thus found, relative to the reference center $\left(X_{r e f}\left(z_{h}\right), Y_{r e f}\left(z_{h}\right)\right)$, was considered as 2D polar origin motion, and was subtracted from the displacements $\left(u_{x}, u_{y}\right)$. The remaining displacements of the points were decomposed into radial $\left(u_{r}\left(\mathbf{x}_{h p}\right)\right)$ and circumferential $\left(u_{\phi}\left(\mathbf{x}_{h p}\right)\right)$ displacements:

$$
\begin{aligned}
& u_{r}\left(\mathbf{x}_{h p}\right)=\sqrt{\left(x_{h p}+u_{x}\left(\mathbf{x}_{h p}\right)-X_{r e j}\left(z_{h}\right)-\Delta X\left(z_{h}\right)\right)^{2}+\left(y_{h p}+u_{y}\left(\mathbf{x}_{h p p}\right)-Y_{r e f}\left(z_{h}\right)-\Delta Y\left(z_{h}\right)\right)^{2}} \\
& -R_{r e f}\left(z_{h}\right) ; \quad p=1,2,3,4 ; h=2,3 \\
& u_{\phi}\left(\mathbf{x}_{h p}\right)=\operatorname{atan} 2\left(y_{h p}+u_{y}\left(\mathbf{x}_{h p}\right)-Y_{0}\left(z_{h}\right)-\Delta Y\left(z_{h}\right), x_{h p}+u_{x}\left(\mathbf{x}_{h p}\right)-X_{0}\left(z_{h}\right)-\Delta X\left(z_{h}\right)\right) \\
& -\phi_{p} ; \quad p=1,2,3,4 ; h=2,3
\end{aligned}
$$

and

$$
u_{\phi}\left(\mathbf{x}_{1 p}\right)=u_{\phi}\left(\mathbf{x}_{2 p}\right), u_{\phi}\left(\mathbf{x}_{4 p}\right)=u_{\phi}\left(\mathbf{x}_{3 p}\right) ; \quad p=1,2,3,4
$$


Next, radial displacements of the points at $z=z_{1}$ and $z=z_{4}$ were calculated as:

$$
u_{r}\left(\mathbf{x}_{h p}\right)=\Delta R\left(z_{h}\right) ; \quad p=1,2,3,4 ; h=1,4
$$

Longitudinal motion was considered linear with $z_{h}$ for a given constant $\phi_{p}$ :

$$
u_{i}\left(\mathbf{x}_{h r}\right)=u_{i}\left(\mathbf{x}_{3 p}\right)+\left(u_{z}\left(\mathbf{x}_{2 p}\right)-u_{z}\left(\mathbf{x}_{, p}\right)\right) \frac{z_{h}-z_{3}}{z_{2}-z_{3}} ; \quad p=1,2,3,4, h=1,4
$$

\section{Appendix C: motion of an arbitrary point in the wall}

Motion of an arbitrary point $\mathbf{x}_{\text {ref }}$ in the left ventricular wall was determined from the motion of the 4 nearest points $x_{h p}$ and the motion of the 2 corresponding polar origins by interpolation of radial, circumferential, and longitudinal displacements, and displacement of the polar origin of the cross-section. The circumferential position of the point $\mathbf{x}_{r e f}$ is:

$$
\phi_{r e f}=\operatorname{atan} 2\left(y_{r e f}-Y_{r e f}\left(z_{r e f}\right), x_{r e f}-X_{r e f}\left(z_{r e f}\right)\right)
$$

To enable bilinear interpolation, the relative angle $\phi_{r e l}$ and the relative height $z_{\text {rel }}$ of the point $\mathbf{x}_{\text {ref }}$ were defined as:

$$
\phi_{r e t}=\frac{\phi_{r e f}-\phi_{a}}{\phi_{b}-\phi_{a}}, \quad z_{r e l}=\frac{z_{r e f}-z_{c}}{z_{a}-z_{c}}
$$

where the subscripts a, b, c, and d denote the upper left, upper right, lower left, and lower right points $\mathbf{x}_{h y}$ that were used for the interpolations, respectively, as indicated in figure 5.C. Then it holds: 


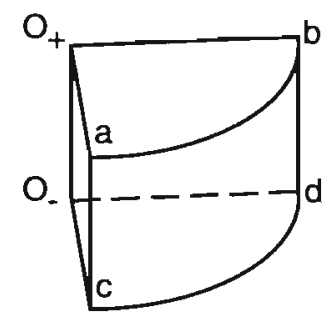

Figure 5.C Indication of the 4 points $(a, b, c$, and d) used for bilinear interpolation. The points

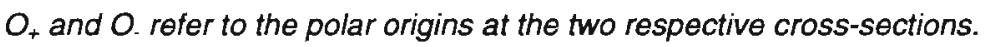

$$
\begin{aligned}
& \left(\begin{array}{l}
u_{r}\left(\mathbf{x}_{r e f}\right) \\
u_{\phi}\left(\mathbf{x}_{r e f}\right) \\
u_{i}\left(\mathbf{x}_{r e f}\right)
\end{array}\right)=\left(\begin{array}{llll}
u_{r}\left(\mathbf{x}_{a}\right) & u_{r}\left(\mathbf{x}_{b}\right) & u_{r}^{*}\left(\mathbf{x}_{c}\right) & u_{r}\left(\mathbf{x}_{d}\right) \\
u_{\phi}\left(\mathbf{x}_{a}\right) & u_{\phi}\left(\mathbf{x}_{b}\right) & u_{\phi}\left(\mathbf{x}_{c}\right) & u_{\phi}\left(\mathbf{x}_{d}\right) \\
u_{z}\left(\mathbf{x}_{a}\right) & u_{z}\left(\mathbf{x}_{b}\right) & u_{i}\left(\mathbf{x}_{c}\right) & u_{z}\left(\mathbf{x}_{d}\right)
\end{array}\right) \cdot \mathbf{A}_{\phi} \cdot \mathbf{A}_{i}, \text { with } \\
& \mathbf{A}_{\phi}=\left(\begin{array}{cc}
\left(1-\phi_{r e l}\right) & 0 \\
\phi_{r e l} & 0 \\
0 & \left(1-\phi_{r e l}\right) \\
0 & \phi_{r e l}
\end{array}\right), \mathbf{A}_{z}=\left(\begin{array}{c}
z_{r e l} \\
\left(1-z_{r e l}\right)
\end{array}\right) \text {, and } \\
& u_{r}^{*}\left(\mathbf{x}_{a, d}\right)=u_{r}\left(\mathbf{x}_{a, d}\right)+\Delta r_{m w} \cdot g_{r}, \text { with } \\
& \Delta r_{r w w}=\sqrt{\left(x_{r e f}-X_{r e f}\left(z_{r e f}\right)\right)^{2}+\left(y_{r e f}-Y_{r e f}\left(z_{r e f}\right)\right)^{2}}-R_{r e f}\left(z_{r e f}\right),
\end{aligned}
$$

with, $\Delta r_{m w}$ the radial distance of the point $\mathbf{x}_{r e f}$ to the mid-wall surface.

For subsequent calculation of $\mathbf{x}_{1}$ in Cartesian coordinates (equation 7), crosssectional motion $\left(\Delta X\left(z_{r f}\right), \Delta Y\left(z_{r e}\right)\right)$ of the polar origin at $z_{\text {ref }}$ was calculated using linear interpolation with respect to $z_{\text {ref }}$.

\section{Appendix D. calculation of strains}

Circumferential $\left(\varepsilon_{\phi}\right)$, longitudinal $\left(\varepsilon_{\varepsilon}\right)$, and surface area $\left(\varepsilon_{\text {area }}\right)$ strains were calculated as follows. For the upper (+) and lower (-) parts of each region (figure 5.4) separately the $\operatorname{arcs}(\Phi)$, and the average radii $(r)$ and heights $(z)$ were computed at the reference time ( $r e f)$ and at time $t$ : 


$$
\begin{array}{ll}
r_{r f}^{+}=R_{r e f}\left(z_{a}\right), & r_{i}^{+}=r_{r f}^{+}+\left(u_{r}\left(\mathbf{x}_{a}\right)+u_{r}\left(\mathbf{x}_{b}\right)\right) / 2, \\
r_{r f}^{-}=R_{r f}\left(z_{c}\right), & r_{t}^{-}=r_{r f}^{-}+\left(u_{r}\left(\mathbf{x}_{c}\right)+u_{r}\left(\mathbf{x}_{d}\right)\right) / 2, \\
\Phi_{r f}^{+}=\frac{1}{2} \pi, & \Phi_{t}^{+}=\Phi_{r e f}^{+}+u_{\phi}\left(\mathbf{x}_{b}\right)-u_{\phi}\left(\mathbf{x}_{a}\right), \\
\Phi_{r f}^{-}=\frac{1}{2} \pi, & \Phi_{t}^{-}=\Phi_{r e f}^{-}+u_{\phi}\left(\mathbf{x}_{d}\right)-u_{\phi}\left(\mathbf{x}_{c}\right), \\
z_{t}^{+}=z_{a}, & z_{i}^{+}=z_{r f}^{+}+\left(u_{z}\left(\mathbf{x}_{a}\right)+u_{z}\left(\mathbf{x}_{b}\right)\right) / 2, \\
z_{r e f}^{-}=z_{c}, & z_{t}^{-}=z_{r f}^{-}+\left(u_{z}\left(\mathbf{x}_{c}\right)+u_{z}\left(\mathbf{x}_{d}\right)\right) / 2,
\end{array}
$$

where the subscripts $a, b, c$, and d denote the upper left, upper right, lower left, and lower right points of the region, respectively (figure 5.A). Subsequently,

$$
\begin{aligned}
& \varepsilon_{\phi}=\frac{\left(\Phi_{t}^{+} \cdot r_{t}^{+}+\Phi_{t}^{-} \cdot r_{t}^{-}\right)-\left(\Phi_{r e f}^{+} \cdot r_{r e f}^{+}+\Phi_{r e f}^{-} \cdot r_{r e f}^{-}\right)}{\left(\Phi_{r e f}^{+} \cdot r_{r f}^{+}+\Phi_{r e f}^{-} \cdot r_{r e f}^{-}\right)} \\
& \varepsilon_{z}=\frac{\left(z_{t}^{+}-z_{t}^{-}\right)-\left(z_{r e f}^{+}-z_{r e f}^{-}\right)}{z_{r f}^{+}-z_{r f}^{-}} \\
& \varepsilon_{\text {area }}=\varepsilon_{c}+\varepsilon_{z}+\varepsilon_{c} \cdot \varepsilon_{\mathrm{z}} .
\end{aligned}
$$


Chapter 6

General discussion 


\subsection{INTRODUCTION}

Various cardiac pathologies are associated with disturbances in cardiac wall motion. Experimental studies have shown the feasibility of Magnetic Resonance (MR) cardiac tagging to assess noninvasively three-dimensional (3D) tissue motion and deformation quantitatively throughout the cardiac wall. This property makes the MR cardiac tagging technique a promising extension of the current cardiac diagnostic tools. For instance, by means of MR cardiac tagging it could be demonstrated that the left ventricular wall undergoes complex 3D nonhomogeneous deformations in myocardial infarction (Kramer et al., 1996) and hypertrophic cardiomyopathy (Young et al., 1994). The time consuming procedure to be followed to perform a full quantitative analysis of the data (McVeigh, 1998), however, have prevented routine clinical use of MR cardiac tagging up to now. Furthermore, clinical interpretation of the large amount of quantitative data obtained from local strain measurements is difficult. The main goal of the studies performed in this thesis was the development of highly automated techniques to analyze MR tagged images and to represent the thus obtained motion information such that it can be interpreted easily.

\subsection{MAJOR RESULTS}

Highly automated methods were developed to quantify cardiac wall deformation by means of MR cardiac tagging. Manual user-interference was limited to delineation of left ventricular wall contours at a single reference time. First, a method to automatically and objectively detect tags was developed and evaluated (chapters 2 and 3). The method was based on band-pass filtering and subsequent thresholding of images. It was demonstrated that the method can be used to assess $2 \mathrm{D}$ cardiac wall deformation in humans. Next, a method was developed to determine 3D cardiac wall motion from simultaneous image cross-correlations in a multiple of cross-sectional views (chapters 4 and 5). With this method, the deformed tagging pattern was deformed back to its initial undeformed state, for all considered moments in time. Thus, the information of $2 D$ images was merged directly into 3D motion information. Furthermore, the shape of the tags was not critical. The method appeared feasible in healthy volunteers and patients with a myocardial infarction. 
Analysis of tagging data was facilitated with the use of kinematic models. The regular $3 \mathrm{D}$ motion patterns occurring in the healthy left ventricle were quantified. In particular, the relation between torsion and volume ejection during the ejection phase of the cardiac cycle was investigated. The relation observed was close to what was predicted by a mathematical model of left ventricular mechanics (Arts et al., 1979), and also close to the one found earlier in experiments on animals (Arts et al., 1984). Since equilibria of forces in the wall were essential to the mathematical model, in the human heart the same equilibria are likely to play an important role.

Next, the underlying kinematic models were modified to deal with 3D wall motion in pathological hearts, i.e., hearts with inhomogeneous contraction patterns. To this purpose more degrees of freedom were added to the kinematic models. With the use of the tag detection method an asymmetrical cross-sectional motion pattern could be detected in patients with a myocardial infarction. The 3D correlation-based method enabled localization of the dysfunctional region in patients.

\subsection{DISCUSSION}

\subsubsection{Quantification of wall motion}

Most methods to quantify cardiac wall motion by means of MR cardiac tagging rely on tag detection algorithms that require delineation of wall contours in all images. Up to now wall contours could not be delineated fully automatically (McVeigh, 1998). It requires a lot of user-guided processing time. For example, if images are acquired from ten short-axis and six long-axis cross-sections through the heart at twelve moments during the cardiac cycle, delineation of wall contours can take up to two hours. Therefore, we changed to a different strategy, in which wall contours only need to be known at one reference time. To this purpose, wall contours are considered to follow the motion of the tags, which is described in the kinematic model. In chapters 2 and 3 corresponding tags detected in consecutive images are identified by an earlier developed procedure (Muijtjens, 1995). For further processing, wall contours need to be known at one given reference time for selection of only those tags tracks that are inside the left ventricular wall. Regarding the later developed correlation-based methods described in chapters 4 and 5, the boundaries of the left ventricular wall are part of the kinematic analysis, such that also for these methods wall segmentation 
has to be performed in only one frame in time. Thus, user-interference time was reduced considerably as compared to earlier described methods to analyze MRtagged images.

The highly automated tag detection method described in chapters 2 and 3 is based on separation of the tags from the background by thresholding of the bandpass filtered images. Tag positions are calculated as the centers of gravity of the tags in the thresholded images. An important advantage of our tag detection method is that it is objective. Employing other methods (Guttman et al., 1994; Kumar and Goldgof, 1994; Kraitchman et al., 1995), full automation of tag detection appears to be difficult. Generally additional user-interventions are needed, increasing subjectivity in the analysis. A disadvantage of the standard thresholding technique we used is that much of the image information is ignored, causing a reduction of spatial accuracy. Our tag detection could probably be improved by employing more sophisticated thresholding techniques, for instance based on local histogram analysis (Jähne, 1993), or by calculating tag positions taking into account the image gray values. However, we chose to abandon the idea of thresholding in favor of the image correlation approach. In chapters 4 and 5 cardiac wall motion is determined directly from the images, without the need to detect individual tags. Tagging is mainly used to provide sufficient contrast within the images. This approach is also advantageous in that a larger fraction of the image information is used for motion analysis, thus improving the signal to noise ratio.

Using tag detection methods, often the strategy is followed to first determine the $2 \mathrm{D}$ motion of all individual tags in all cross-sections, merge this information into local 3D wall motion, and subsequently combine the local wall motion over larger regions to describe 3D wall motion on a larger scale (Young et al., 1994; O'Dell et al., 1995; Kramer et al., 1996; Park et al., 1996). Also optical flow methods (Prince and McVeigh, 1992), which do not require detection of individual tags, follow a similar strategy. This strategy is not optimal because for quantification of a limited number of relevant motion parameters a large amount of local displacements has to be estimated first. Using the image correlation method, we perform the motion estimation with the reduced set of relevant parameters directly over all images simultaneously. Then, the parameter values are expected to be estimated statistically more accurately. 
To compare the results of tag detection with those of image correlation, we determined cross-sectional area ejection and rigid body rotation and displacement using both approaches. Results from two short-axis cross-sections were combined to calculate torsion. In chapter 3 it was shown that due to fading of the tags the determined time course of individual tag motion could not cover the entire ejection phase of the cardiac cycle. This problem was solved by recording two MRI sequences in time that were partly overlapping. The time course of deformation during the entire ejection phase was obtained by connecting the separately measured parts of the time course. By means of the image correlation approach motion parameters could be estimated accurately for about $80-100 \mathrm{~ms}$ (4 to 5 image frames) further in time, so that often the entire ejection phase was covered. Differences in the torsion to area ejection ratio obtained with both approaches were less than $3 \%$. Thus, the correlation methods have shown to be accurate and less sensitive to effects of noise in the images.

\subsubsection{Clinical application of MR cardiac tagging}

In many MR cardiac tagging studies (Kramer et al., 1993; Young et al., 1994; Azhari et al., 1995; Lima et al., 1995; O'Dell et al., 1995) the focus is on quantifying local strains from the images. This is a fruitful approach, especially when using local wall mechanics to differentiate healthy from diseased tissue. To aid clinical diagnosis local strain parameters can be displayed by pseudocolors overlaid on the original images or on a 3D reconstruction of cardiac geometry (Axel et al., 1995; McVeigh, 1996). A dynamic display shows $3 \mathrm{D}$ global patterns of wall motion, which enables clinicians to evaluate motion disorders (McVeigh, 1998). We followed a different approach. An important aspect of the present study is that we focused on quantifying the characteristic patterns of motion occurring in the healthy or pathologic left ventricular wall. Often, for a particular pathology, specific patterns in motion are to be expected. Foreknowledge about expected aberrations in motion may be used to enhance sensitivity in detecting disorders. Therefore, we have chosen to describe the global motion patterns first and thereafter to zoom in on a more detailed description of the aberrations using a kinematic model. Currently, our spatial resolution is still less than that of others (Young and Axel, 1992; O'Dell et al., 1995), but this is open to improvement by using their more advanced MR-techniques. 
As a first step in the quantitative description of aberrant motion patterns using a limited number of parameters, we quantified the influence of myocardial infarction by a sinusoidal course of area strain values along the circumference, sampled at four points. Thus, the circumferential location of the infarction was well approximated (chapter 5). To describe the extent and severity of the infarction in more detail, which is necessary for clinical purposes, the spatial resolution should be increased by sampling more points, both along the circumferential and longitudinal directions. In conjunction, the kinematic description of the infarction has to be extended to more versatile functions, describing the shape, size and amplitude of the aberrant motion pattern by a limited number of parameters. A tangential spatial resolution of about 10 $\mathrm{mm}$ may be sufficient because the width of the hypokinetic transition zone around an ischemic region is about $20 \mathrm{~mm}$ (Gallagher et al., 1987; Prinzen et al., 1989).

A characteristic global measure of cardiac performance may be the torsion to volume ejection ratio, which we investigated in chapters 3 and 4 . In the healthy left ventricle all myocardial fibers are known to shorten approximately to the same extent during the ejection phase. In a mathematical model of left ventricular mechanics it was found that this uniformity requires that torsion around the long axis is a unique function of the ratio of cavity volume to wall volume (Arts et al., 1979; Arts et al., 1984). In the present study we found that in healthy volunteers this relationship was satisfied. When fiber shortening becomes non-uniform due to pathology, the torsion to volume ejection ratio may change. For instance, on theoretical grounds the torsion to volume ejection ratio is expected to increase in the presence of subendocardial dysfunction. In this respect it should be noted that Magnetic Resonance cardiac tagging is a very suitable tool to measure torsion, more suitable than other, more conventional techniques such as echocardiography.

\subsubsection{Future perspectives}

In the present thesis we showed the feasibility of the developed image correlation method to quantify 3D cardiac wall deformation using MR cardiac tagging in a highly automated way. Before the method can be applied in a routine clinical setting, however, a number of improvements have to be made. The method should be extended to analyze MR-tagged images from a larger number of cross-sections 
(Young and Axel, 1992; O'Dell et al., 1995), requiring a more detailed kinematic model than the one in chapter 5 . This would allow to obtain a higher spatial resolution to detect regions of motional dysfunction as compared to the current study. In addition, the period of motion analysis may be extended over a larger part of the cardiac cycle by more advanced tagging and imaging techniques, such as CSPAMM (Fischer et al., 1993), breath-hold cine MRI (McVeigh and Atalar, 1992) or echoplanar imaging (Tang et al., 1995), with which tagging contrast can be enhanced considerably. The image correlation method we proposed can also be employed in combination with any of these techniques. For instance, there is no principle limitation to apply our method to line tagged images instead of images with a tagging grid. Tagging is mainly used to provide sufficient contrast in the images. The proposed methods should be compared with more established methods to analyze MR-tagged images (Young and Axel, 1992; O'Dell et al., 1995) regarding accuracy of motion estimates and user-interference time needed. Also, a more thorough investigation is needed of the reproducibility of results obtained using the image correlation approach.

Although relative to echocardiography MR cardiac tagging enables a much more detailed assessment of cardiac wall deformation, the advantages for routine clinical diagnosis are yet to be investigated. When the motion aberrations are very pronounced, such as in severe myocardial infarction, there is less need for an extensive quantitative analysis and MR cardiac tagging probably has no additional value. In these cases echocardiography generally yields sufficient diagnostic possibilities and is preferable to the much more expensive MR techniques. However, when the motion aberrations are subtle, such as in detection of a subendocardial infarction, or in differentiation between stunned but viable tissue from infarcted tissue in a low-dose dobutamine stress-test (Croisille et al., 1999), there is need for a proper quantitative analysis. In these cases MR cardiac tagging is probably preferable to investigate the aberrant motion patterns. An important advantage of the methods developed in the present thesis is that they are highly automated, contributing to the routine clinical applicability of MR cardiac tagging.

\subsection{CONCLUSIONS}

1. Methods were developed which enable quantification of $3 \mathrm{D}$ cardiac wall deformation in a highly automated way. This automation was reached by limiting 
user-interference to segmentation of the cardiac wall in imaged cross-sections at one single reference time.

2. The developed image correlation method is flexible in regard to the specific tagging technique used (tag shape, image quality/contrast) and the desired spatial resolution of the motion field, which is associated with the number and locations of the cross-sections through the heart.

3. Complex wall deformation can be described by a limited number of kinematic parameters, forming the basis of characteristic motion patterns. Recognition of these patterns may facilitate diagnosis in routine clinical application.

4. The methods can be applied in healthy volunteers as well as in patients with inhomogeneously contracting hearts.

\section{REFERENCES}

Arts, T., Meerbaum, S., Reneman, R. S., and Corday, E. (1984) Torsion of the Left Ventricle during the Ejection Phase in the Intact Dog. Cardiovasc Res 18, 183-193.

Arts, T., Reneman, R. S., and Veenstra, P. C. (1979) A model of the mechanics of the left ventricle. Ann Blomed Eng 7, 299-318.

Axel, L., Bloomgarden, D. C., Chang, C.-N., Fayad, Z. A. Kraitchman, D. L., and Young, A. A. (1995) An integrated program for 2-D and 3-D analysis of heart wall motion from Magnetic Resonance Imaging. In Computers in Cardiology. Vienna: IEEE.

Azhari, H., Weiss, J. L., Rogers, W. J., Siu, C. O., and Shapiro, E. P. (1995) A noninvasive comparative study of myocardial strains in ischemic canine heart using tagged MRI in 3-D. Am J Physiol 268, H1918-H1926.

Croisille. P., Moore, C. C., Judd, R. M., Lima, J. A. C., Arai, M., McVeigh, E. R., Becker, L. C., and Zerhouni, E. A. (1999) Differentiation of viable and nonviable myocardium by the use of threedimensional tagged MRI in 2-day-old reperfused canine infarcts. Circulation $\mathbf{9 9}$, 284-291.

Fischer, S. E., McKinnon, G. C., Maier, S. E., and Boesiger, P. (1993) Improved Myocardial Tagging Contrast. Magn Reson Med 30, 191-200.

Gallagher, K. P., McClanhan, T. B., Lynch, M. J., Bolling. S. F., and Dunharn, W. R. (1987) Occlusion of the left anterior descending artery produces a larger functional border zone than circumflex occlusion. Circulation 76(SuppI. IV), 373.

Guttman, M. A., Prince, J. L., and McVeigh, E. R. (1994) Tag and contour detection in tagged MR images of the left ventricie. IEEE T Med Imaging 13, 74-88.

Jăhne, B. (1993) Digital Image Processing Concepts, Algorithms and Scientific Applications. Springer-Verlag, Berlin.

Kraitchman, D. L, Young, A. A., Chang, C.-N., and Axel, L. (1995) Semi-automatic 
tracking of myocardial motion in MR-tagged images. IEEE T Med Imaging 14, 422-433.

Kramer, C. M., Lima, J. A. C., Reichek, N., Ferrari, V. A., Laneras, M. R., Palmon, L. C., Yeh, L.T., Tallant, B., and Axel, L. (1993) Regional differences in function within noninfarct myocardium during left ventricular remodeling. Circulation $\mathbf{8 8}$, 1279-1288.

Kramer, C. M., Rogers, W. J., Theobald, T. M., Power, T. P., Petruolo, S., and Reichek, N. (1996) Remote noninfarcted region dysfunction soon after first anterior myocardial infarction. A Magnetic Resonance Tagging study. Circulation 94, 660-666.

Kumar, S. and Goldgof, D. (1994) Automatic tracking of SPAMM grid and the estimation of deformation parameters from cardiac MR images. IEEE T Mod Imaging 13, 122-132.

Lima, J. A. C., Ferrari, V. A., Reichek, N., Kramer, C. M., Palmon, L., Llaneras, M. R., Tallant, B., Young, A. A., and Axel, L. (1995) Segmental motion and deformation of transmurally infarcted myocardium in acute postinfarct period. Am J Physiol 268, H1304-H1312.

McVeigh, E. R. (1996) MFil of myocardial function: motion tracking techniques. Magn Reson Imaging 14, 137-150.

McVeigh, E. P. (1998) Regional myocardial function. Cardiac Magnetic Resonance 16, 189-205.

McVeigh, E. R. and Atalar, E. (1992) Cardiac Tagging with Breath-Hold Cine MRI. Magn fies Med 28, 318-32?.

Muiltiens, A. M. M. (1995) The reconstruction of 3-D marker trajectories in measuring heart motion. Ph.D. thesis, Maastricht University.

O'Dell, W. G., Moore, C. C., Hunter, W. C., Zerhouni, E. A., and McVeigh, E. R. (1995) Three-dimensional myocardial deformations: calculation with displacement field fitting to tagged MR images. Radiology 195, 829-835.

Park, J., Metaxas, D., and Axel, L. (1996) Analysis of left ventricular wall motion based on volumetric deformable models and MRI-SPAMM. Med. Image Anal. 1, 53 71.

Prince, J. L. and McVeigh, E. R. (1992) Motion estimation from tagged MR image sequences. IEEE T Med Imag 11, 238-249.

Prinzen, F. W., Arts, T., Hoeks, A. P. G., and Reneman, A. S. (1989) Discrepancies between Myocardial Blood Flow and Fiber Shortening in the Ischemic Border Zone as assessed with Video Mapping of Epicardial Deformation. Eur J of Physiology 415, 220229.

Tang, C., McVeigh, E. R., and Zerhouni, E. A. (1995) Multi-shot EPI for improvement of myocardial tagging contrast: comparison with segmented SPGR. Magn Res Med 33, 443-447.

Young, A. A. and Axel, L. (1992) Threedimensional motion and Deformation of the Heart Wall: Estimation with Spatial Modulation of Magnetization - A ModelDasecl Approach. Aadiology 185, 241-247.

Young, A. A., Kramer, C. M. Ferrari, V. A., Axel, L., and Reichek, N. (1994) Threedimensional left ventricular deformation in hypertrophic cardiomyopathy. Circulation 90, 854-867. 


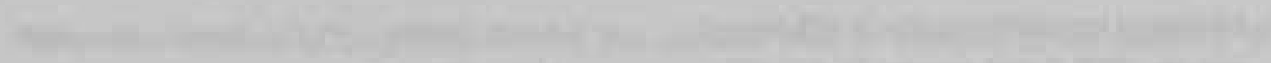
$-1$

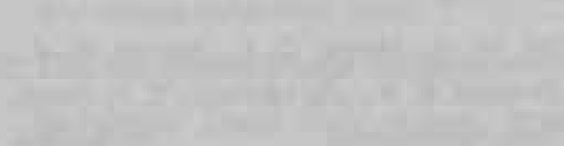

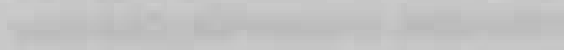

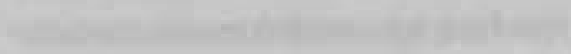

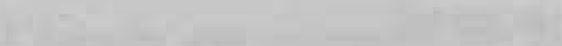

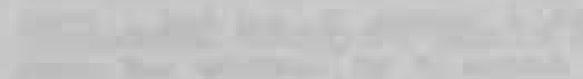
(1)

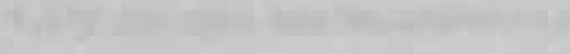

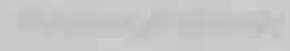

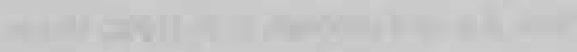

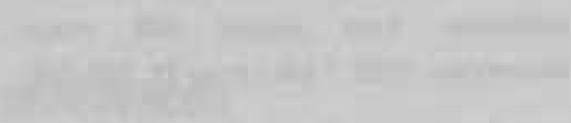

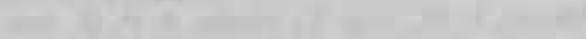

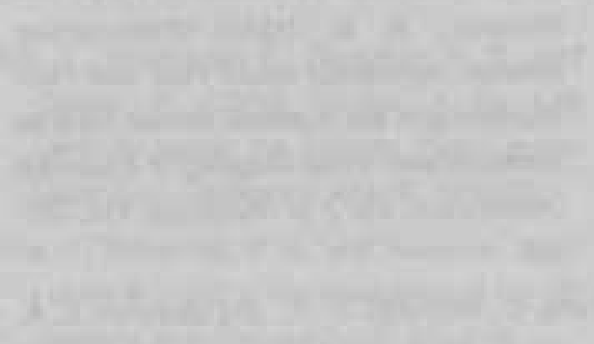

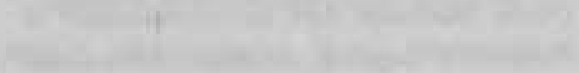

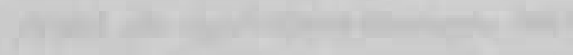

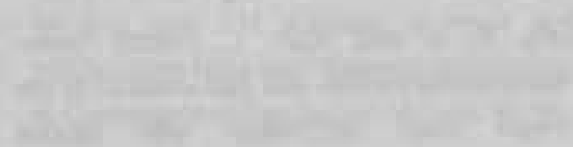

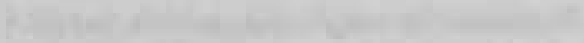

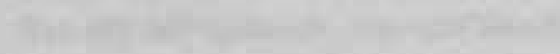

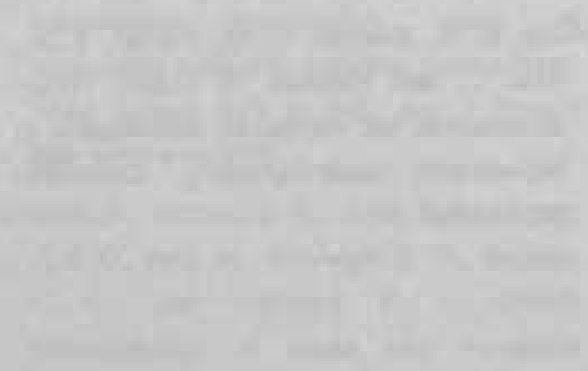

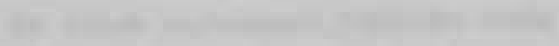

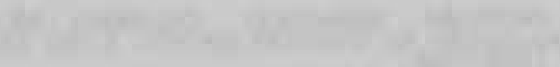

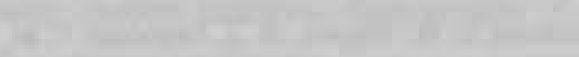

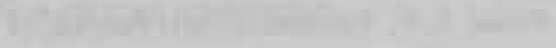

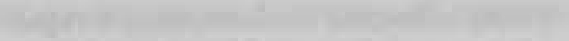

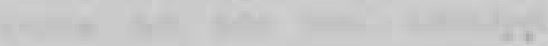

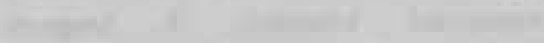

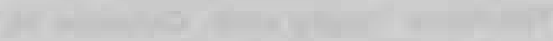
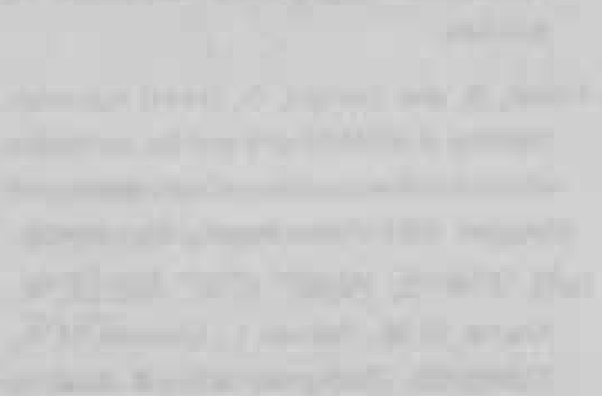

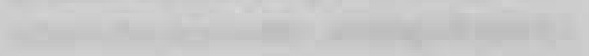
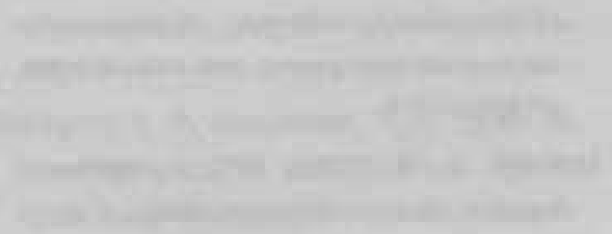

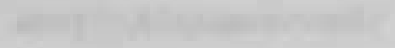
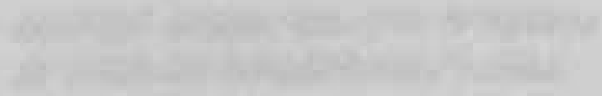

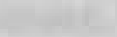

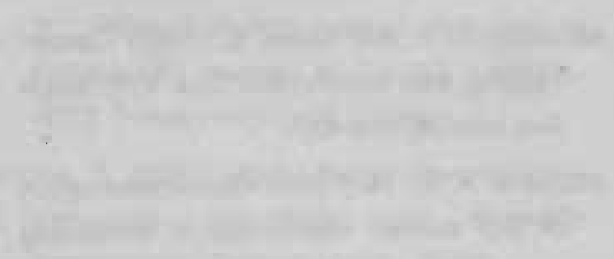




\section{Summary}

\section{Samenvatting}




\section{Summary}

During the cardiac cycle the wall of the left ventricle deforms in a complex manner. During the ejection phase, the heart pumps blood into the aorta, resulting in a decrease of cavity volume combined with thickening of the wall. In addition, torsion of the wall occurs. Torsion is represented by a base to apex gradient of circumferential rotation around the long axis of the left ventricle. This mode of deformation allows for homogeneity of fiber strains and stresses throughout the wall.

Cardiac pathologies such as myocardial infarction or ischemia, abnormal impulse conduction, and hypertrophic cardiomyopathy strongly influence deformation of the left ventricle during the cardiac cycle. Therefore, methods to measure cardiac deformations during the carctiac cycle, and changes thereof in disease, have become important tools in clinical diagnosis. Currently, echocardiography and Magnetic Resonance Imaging (MRI) are used clinically to analyze motion and deformation of heart wall contours. More recently, the advent of Magnetic Resonance (MR) tagging enables noninvasive quantitative analysis of three-dimensional (3D) tissue deformation throughout the heart wall. Using this technique, a pattern of tags is attached noninvasively to the cardiac tissue by local alteration of tissue magnetization. The tagging pattern deforms along with the tissue. Visualization of the tags in MR images provides information on motion and deformation of the cardiac walls. However, clinical application of the technique is significantly impeded because analysis of the acquired images is still laborious. Also, the reconstruction of $3 \mathrm{D}$ deformation from multiple cross-sections through the heart is far from optimal.

The general objective of the studies presented in this thesis was the development of a method to quantify 3D left ventricular wall motion from MR-tagged images that is automated to a high degree. Moreover, the method should be flexible in regard to the specific shape of the tags, the number of image cross-sections through the heart, and the specific locations of these cross-sections. We also tried to present the motion information in such a way that it can be easily interpreted by a physician. To accomplish this, we incorporated foreknowledge about expecteci characteristics of left ventricular wall motion into the analysis. This foreknowledge was represented in a kinematic model, which described wall motion by a limited number of characteristic motion patterns. 
First, we focused on quantification of left ventricular wall motion from MR-tagged images of a single cross-section. To this purpose, tags were detected and followed in time (chapters 2 and 3). Most of the methods developed by other groups are also based on this approach, with the distinction that we concentrated on automating the methods. In the later studies (chapters 4 and 5), methods were improved and extended to determine 3D wall motion. To this purpose, MR-tagged images from multiple cross-sections were analyzed simultaneously. The need to detect individual tags was avoided using pattern recognition and image cross-correlation techniques.

In chapter 2 a method is presented to detect and follow tags in MR-tagged images automatically. To this purpose, techniques were adapted which hadi been employed earlier in the analysis of video images of optical markers on the epicardial surface. Manual user-interference was limited to delineation of left ventricular wall contours in a single reference frame. The thus found tag tracks were fitted to a kinematic model of cross-sectional motion. The used kinematic model contained the regular modes of cross-sectional motion (area ejection, rotation, and rigid body displacement), supplemented with a two-parameter description of the motion component related to a regional dysfunction. For this description the knowledge was used that, according to finite element models of the mechanics of the infarcted heart, an infarcted region causes motional asymmetry extending far beyond the infarct boundary. MR-tagged images of one short-axis cross-section were acquired in 9 healthy volunteers and in 5 patients with myocardial infarction. Cross-sectional rotation and the decrease in cross-sectional area of the cavity were both found to be greater in the volunteers than in the patients: $6.4 \pm 1.5$ vs. $3.0 \pm 0.8$ degrees (mean \pm sd; $p<0.001$ ), and $945 \pm 71$ vs. $700 \pm 176 \mathrm{~mm}^{2}$ ( $\left.p=0.02\right)$, respectively. In the patients, asymmetry of wall motion, as expressed by a sine wave dependency of contraction around the circumference, was significantly enlarged $(p=0.02)$. It was concluded that the proposed method of kinematic analysis can be used to assess cross-sectional cardiac deformation in humans. Of course, for an accurate description of the influence of myocardial infarction, the analysis needs to be extended to use information on more crosssections.

Previously, in a mathematical model of left ventricular mechanics it was found that torsion around the long axis was uniquely related to the ratio of cavity volume to wall 
volume, in order to homogenize fiber strains throughout the wall during the ejection phase. In order to verify this prediction, in chapter 3 the above-described method for analysis of MR-tagged images was extended to enable determination of torsion of the left ventricle. MR-tagged images from two parallel short-axis cross-sections were acquired in 9 healthy volunteers. Tags were detected and followed for each crosssection independently, as described in chapter 2. Torsion was calculated from the difference in rotation between the two cross-sections. Contraction was quantifiecl as the change in the logarithm of the ratio of summed cavity area to summed wall area of the two cross-sections. Linear regression was applied to torsion as a function of contraction. The slope thus found $(-0.173 \pm 0.024 \mathrm{rad}$, mean $\pm \mathrm{sd})$ was in agreement with predictions by the earlier presented model of left ventricular mechanics $(-0.194 \pm 0.026 \mathrm{rad})$. Since equilibria of forces in the wall were essential to this model, in the real heart these equilibria are likely to be the main determinants of physiologic cardiac motion.

In chapter 4 the step was made towards a full 3D kinematic analysis. MR-tagged images were acquired from two short-axis cross-sections and two perpendicular longaxis cross-sections in 10 healthy volunteers. A previously developed 3D kinematic model was used to describe the normal pattern of global left ventricular wall motion. Parameters in the kinematic model were determined from the images using image cross-correlations rather than detection of individual tags. More specifically, using the 3D kinematic model the deformed tagging pattern was deformed back to its initial undeformed state, for all considered moments in time. This was done for all crosssections simultaneously, thus merging the information of multip!e 20 images direct]y/ into 3D motion information. The method is highly automated and to a great extent independent of the shape of the tags. Again, manual user-interference was limited to delineation of left ventricular wall contours at a single reference time. The methoc was shown to work in healthy volunteers. Compared to the tag detection method developed in chapters 2 and 3 , the image correlation method proved to be less sensitive to noise in the images.

In chapter $\mathbf{5}$ a kinematic model was developed that allows for a description of a 3D motion field with regional abnormalities. Again, MR-tagged images were acquired from two short-axis cross-sections and two mutually perpendicular long-axis crosssections. The parameters in the kinematic model were estimated in a similar manner 
as in chapter 4 . The number of motion modes allowed was extended to determine left ventricular wall thickening and circumferential, longitudinal, and area strains. Measurements were performed in 9 healthy volunteers as well as in 2 patients with myocardial infarction. Strain values in the volunteers were within the normal physiological range. In both patients the location of the region of diminished area strain coincided with the clinically determined location of the infarction. Manual userinterference time was approximately 10 minutes per subject. We conclude that the developed image correlation method allows for quantification of inhomogeneous $3 \mathrm{D}$ cardiac wall deformation by means of MR tagging in a highly automated way. For better clinical application, it is recommended that the spatial resolution be increased by acquiring images from more cross-sections through the heart. In conjunction, the kinematic model should be extended to allow for the higher spatial resolution.

The following conclusions may be formulated on the basis of the studies presented in this thesis:

1. We succeeded in developing methods to quantify $3 D$ cardiac wall deformation in a highly automated way. User-interference was limited to segmentation of the cardiac wall in imaged cross-sections at one single reference time.

2. The developed image correlation method is flexible in regard to the specific tagging technique used (tag shape, image quality and contrast) and the desired spatial resolution of the motion field, which is associated with the number and locations of the cross-sections through the heart.

3. Complex wall deformation can be described by a limited number of kinematic parameters, forming the basis of characteristic motion patterns. Recognition of these patterns may facilitate diagnosis in routine clinical application.

4. In the healthy human left ventricle torsion around the long axis and the ratio of cavity volume to wall volume are uniquely related during the ejection phase. This unique relation is connected with the homogeneous distribution of fiber strains and stresses throughout the left ventricular wall.

5. The methods can be applied in healthy volunteers as well as in patients, with inhomogeneously contracting hearts. 


\section{Samenvatting}

Gedurende de hartcyclus vervormt de wand van het linker ventrikel van het hart op een complexe manier. Tijdens de ejectiefase pompt het hart bloed in de aorta, waarbij het holtevolume van het linker ventrikel afneemt en de wand dikker wordt. Tegelijkertijd ondergaat de wand een torsiebeweging, gekenmerkt door een verschil in rotatie om de lange as tussen basis ("bovenkant") en apex ("onderkant"). Deze vervormingscomponent zorgt voor een homogene belasting van spiervezels in de hartwand.

Hartafwijkingen zoals hartinfarct, ischemie, abnormale impulsgeleiding en hypertrofe cardiomyopathie hebben een sterke invloed op de vervormingen van de linker ventrikelwand gedurende de hartcyclus. Methoden die de vervormingen van de hartwand en veranderingen daarvan kunnen meten zijn daarom een belangrijk hulpmiddel geworden bij de diagnose van hartafwijkingen. In de kliniek wordt momenteel voornamelijk echocardiografie en Magnetic Resonance Imaging (MRI) gebruikt om bewegingen en vervormingen van de contouren van de hartwand te analyseren. Met de komst van de Magnetic Resonance (MR) tagging techniek wordt het mogelijk om noninvasief driedimensionale (3D) weefselvervormingen in de hartwand kwantitatief te analyseren. Met behulp van deze techniek. worct een patroon van tags (= markeerders) noninvasief in het hartweefsel aangebracht door de magnetisatie van het weefsel lokaal te veranderen. Het tagging patroon vervormt vervolgens met het weefsel mee. Visualisatie van de tags in opeenvolgende MR beelden geeft informatie over beweging en vervorming van de hartwand. Klinische toepassing van de methode wordt momenteel beperkt doordat de analyse van de verkregen beelden erg tijdrovend is. Bovendien is de reconstructie van 3D vervorningen uit meerdere $2 \mathrm{D}$ beelddoorsneden nog verre van optimaal.

Het heofddoel van het in dit proetschrift beschreven werk was de ontwikkeling van een methode om 3D vervormingen van de hartwand te kunnen kwantificeren met behulp van MR tagging op een in hoge mate geautomatiseerde manier. De methode behoort flexibel te zijn voor wat betreft de precieze vorm van de tags, het aantal gebruikte beelddoorsneden door het hart en de positionering van deze doorsneden. Voorts is getracht om de bewegingsinformatie zodanig te presenteren dat dezes gemakkelijk geïnterpreteercl kan worden door een medicus. Om dit te bereiken 
hebben we voorkennis over de te verwachten karakteristieken van linker ventrikel wandbewegingen in de analyse verwerkt. Deze voorkennis is vastgelegd in een zogenaamd kinematisch model, waarin wandbewegingen worden beschreven aan de hand van een beperkt aantal karakteristieke bewegingspatronen.

Het werk is begonnen met de ontwikkeling van een methode voor kwantificering van linker ventrikel wandbewegingen met gebruik van slechts één MA tagging doorsnede. Hiertoe werden afzonderlijke tags gedetecteerd en gevolgd in de tijd (hoofdstukken 2 en 3). De tot nu toe gebruikelijke methoden zijn meestal ook gebaseerd op deze aanpak. In het huidige onderzoek is relatief veel aandacht besteed aan automatisering van de methoden. Vervolgens (hoofdstukken 4 en 5) zijn de methoden verbeterd en uitgebreid met de analyse van wandbewegingen in $3 \mathrm{D}$. Om dit te realiseren werden MR tagging beelden van meerdere doorsneden van het linker ventrikel simultaan geanalyseerd. Door toepassing van kruiscorrelatietechnieken bij de bewegingsdetectie in MR beeldreeksen verviel de noodzaak tot het detecteren van individuele tags.

In hoofdstuk 2 is een methode gepresenteerd om tags in MR beelden geautomatiseerd te detecteren en te volgen. Bestaande technieken voor de analyse van videobeelden van optische markeerders op het hartoppervlak zijn hiervoor gemodificeerd. Handmatige handelingen zijn beperkt gebleven tot het aangeven van linker ventrikel wandcontouren in één enkel referentiebeeld. De op deze manier gevonden tag-sporen werden gefit op een kinematisch model dat de $2 \mathrm{D}$ beweging binnen een doorsnede beschrijft. Het gebruikte kinematisch model bevatte naast de reguliere bewegingscomponenten binnen een doorsnede (vermindering van holteoppervlakte, rotatie en verplaatsing) een additionele 2-parameter beschriiving van een aan regionale dystunctie gerelateerde bewegingscomponent. Bij deze beschrijving is voorkennis gebruikt die verkregen is uit simulaties met numerieke modellen van de mechanica van het geïnfarcteerde hart. Hierbij bleek dat een geïnfarcteerd gebied een bewegingsasymmetrie veroorzaakt die zich tot ver buiten de infarctgrens uitstrekt. De ontwikkelde methode van bewegingsanalyse werd getest bij 9 gezonde vrijwilligers en bij 5 patiënten met een hartinfarct. Hierbij werden MR tagging beelden gemaakt van één korte as doorsnede. Potatie en afname van de holte-opperviakte waren beide groter voor de vrijwilligers dan voor de patiênten: $6,4 \pm 1,5$ versus $3,0 \pm 0,8$ graden (gemiddelde \pm standaard deviatie; $p<0,001$ ), 
respectievelijk, $945 \pm 71$ versus $700 \pm 176 \mathrm{~mm}^{2} \quad(p=0,02)$. De asymmetrie van wandbeweging werd uitgedrukt als de sinusoïdale component in de relatie tussen omtreksverkorting en circumferentiële positie op de wand. Bij de patiënten bleek deze maat van asymmetrie van wandbeweging significant vergroot $(p=0,02)$. Geconcludeerd kan worden dat de voorgestelde methode van bewegingsanalyse gebruikt kan worden om hartwandvervormingen binnen een hartdoorsnede te bepalen in mensen. Voor een accurate beschrijving van de invloed van een hartinfarct moet de analyse uitgebreid worden door informatie te benutten van meerdere doorsneden.

Ervan uitgaande dat de belasting van spiervezels in de hartwand homogeen verdeeld is, werd eerder in een mathematisch model van de mechanica van het linker ventrikel gevonden dat er gedurende de ejectiefase een unieke relatie bestaat tussen de hoeveelheid torsie en de verhouding van holtevolume tot wandvolume. Om deze voorspelling te verifiëren is in hoofdstuk 3 de methode van bewegingsanalyse uitgebreid teneinde torsie van het linker ventrikel te kunnen bepalen. MR tagging beelden van twee parallelle korte as doorsneden werden gemaakt bij 9 gezonde vrijwilligers. Tags werden gedetecteerd en gevolgd voor iedere doorsnede apart, zoals beschreven in hoofdstuk 2. Torsie werd bepaald uit het verschil in rotatie tussen de twee doorsneden. Contractie werd gekwantificeerd als de verandering in de logaritme van de verhouding van holte-oppervlakte tot wandoppervlakte van de twee doorsneden. Lineaire regressie, toegepast op torsie als een functie van contractie, resulteerde in een helling van $-0.173 \pm 0.024$ rad. Deze waarde komt goed overeen met voorspellingen door het eerder genoemde model van linker ventrikel mechanica $(-0.194 \pm 0.026 \mathrm{rad})$. Aangezien een gelijke verdeling van mechanische spanningen over de spiervezels in de hartwand essentieel is in dit model, is het waarschijinliik dat ook in het echte hart deze gelijkmatigheid bepalend is voor de vervorming van de hartwand gedurende de hartcyclus.

In hoofdstuk 4 is de stap gemaakt naar een volledig 3D kinernatische analyse. Mierbij hebben we de beschikking over MR tagging beelden van twee korte as doorsneden en twee onderling loodrechte lange as doorsneden. Een eerder ontwikkeld 3D kinematisch model is aangepast om het normale patrcon van globale linker ventrikel wandbewegingen to beschrijven. Parameterwaarden in het model werden bepaald uit de beelden gebruikmakend van beeld-kruiscorrelaties. Er hoefden dus geen individuele tags gedetecteerd te worden. Er werd namelijk gebruik gemaakt 
van het 3D kinematisch model om het vervormde patroon van tags voor alle tijdstippen terug te vervormen naar zijn oorspronkelijke, onvervormde toestand. Dit werd gedaan voor alle beelddoorsneden simultaan, zodat op deze manier informatie van meerdere 2D beelden rechtstreeks werd samengevoegd om de best passende $3 \mathrm{D}$ vervorming van de linker ventrikelwand te bepalen. De methode is in hoge mate geautomatiseerd en onafhankelijk van de precieze vorm van de tags. Handmatige handelingen zijn weer beperkt gebleven tot het aangeven van linker ventrikel wandcontouren op één enkel referentietijdstip. In een experimentele serie van 10 gezonde vrijwilligers werd aangetoond dat de methode werkt. Vergeleken met de tag detectie methode, zoals beschreven in hoofdstukken 2 en 3 , blijkt de beeldcorrelatiernethode minder gevoelig te zijn voor ruis in de beelden.

In hoofdstuk $\mathbf{5}$ is een kinematisch model ontwikkeld dat het mogelijk maakt om een beschrijving te geven van een $3 \mathrm{D}$ bewegingsveld met regionale afwijkingen. Het aantal bewegingscomponenten in het model is hiertoe uitgebreid om wandverdikking en circumferentiële-, longitudinale- en oppervlakterekken to kunnen bepalen. $\mathrm{Er}$ werden weer MR tagging beelden gemaakt van twee korte as doorsneden en twee onderling loodrechte lange as doorsneden. De parameterwaarden in het kinematisch model werden geschat op een vergelijkbare manier als in hoofdstuk 4, dat wil zeggen dat ook hier gebruik gemaakt is van beeld-kruiscorrelaties. Metingen werden uitgevoerd bij 9 gezonde vrijwilligers en in 2 patiënten met een hartinfarct. Rekwaarden waren voor de vrijwilligers binnen het normale fysiologische bereik. In beide patiënten bleek de locatie van het gebied met verminderde oppervlakterek overeen te komen met de klinisch bepaalde locatie van helt infarct. De tijd die nodig was voor handmatige handelingen tijdens de analyse bleef beperkt tot ongeveer 10 minuten per persoon. Geconcludeerd kan worden dat de ontwikkelde beelclcorrelatiemethode geschikt is om inhomogene 3D hartwandvervormingen te kwantificeren met behulp van MA tagging. Voor betere klinische toepassing dient de spatiële resolutie verhoogd te worden door beelden van meer doorsneden door het hart te maken. In combinatie hiermee moet dan ook het kinematisch model uitgebreid worden om de hogere spatiẹle resolutie in beweging te kunnen beschrijuen. 
Naar aanleiding van de in dit proefschrift beschreven onderzoeken zijn de volgende conclusies geformuleerd:

1. We zijn erin geslaagd methoden te ontwikkelen om 3D hartwandvervormingen te kwantificeren met behulp van MR tagging, op een in hoge mate geautomatiseerde manier. Handmatige handelingen zijn beperkt tot het aangeven van linker ventrikel wandcontouren in beelddoorsneden op slechts één enkel referentietijdstip.

2. De ontwikkelde beeldcorrelatiemethode is flexibel voor wat betreft de precieze vorm van de tags, de beeldkwaliteit en de gewenste spatiële resolutie van het bewegingsveld, welke gerelateerd is aan het aantal en de locaties van de beelddoorsneden door de hartwand.

3. Complexe hartwandvervormingen kunnen beschreven worden met behulp van een beperkt aantal kinematische parameters, die de basis vormen van karakteristieke bewegingspatronen. Herkenning van deze patronen kan helpen bij routinematige diagnose van bepaalde hartziekten, zoals bijvoorbeeld een hartinfarct.

4. In het gezonde menselijke linker ventrikel bestaat er gedurende de ejectiefase een unieke relatie tussen torsie om de lange as en de verhouding van volume-ejectie tot wandvolume. Deze relatie wordt bepaald door de homogene verdeling van spiervezelverkorting gedurende de ejectiefase.

5. De ontwikkelde methoden voor bewegingsanalyse kunnen toegepast worden in zowel gezonde vrijwilligers als in patiënten met een inhomogeen contraherend hart. 

